\title{
A Comprehensive Nonlinear Dynamic Model for Ball Screw Feed System With Rolling Joint Characteristics
}

\author{
Mengtao Xu \\ Northeastern University
}

Changyou Li ( $\boldsymbol{D}$ chyli@mail.neu.edu.cn )

Northeastern University https://orcid.org/0000-0003-0076-074X

Hongzhuang Zhang

Northeastern University

Zhendong Liu

Northeastern University

Yimin Zhang

Shenyang University of Chemical Technology

\section{Research Article}

Keywords: ball screw feed system, analytical model, coupling vibration, nonlinear dynamics, multi-body system

Posted Date: June 1st, 2021

DOI: https://doi.org/10.21203/rs.3.rs-551706/v1

License: (c) (1) This work is licensed under a Creative Commons Attribution 4.0 International License. Read Full License 


\title{
A comprehensive nonlinear dynamic model for ball screw feed system with rolling joint characteristics
}

\author{
Mengtao Xu ${ }^{\mathrm{a}}$, Changyou $\mathrm{Li}^{\mathrm{a}^{*}}$, Hongzhuang Zhang ${ }^{\mathrm{a}}$, Zhendong Liu ${ }^{\mathrm{a}}$, Yimin Zhang ${ }^{\mathrm{b}}$ \\ ${ }^{a}$ School of Mechanical Engineering and Automation, Northeastern University, Shenyang, 110819, \\ China \\ b Equipment Reliability Institute, Shenyang University of Chemical Technology, Shenyang, \\ 110142, China
}

\begin{abstract}
Modern tendency of machine tools design requires more accurate model to predict the system dynamics, in order to anticipate its interaction with machining process. In this paper, a comprehensive dynamic model of ball screw feed system (BSFS) considering nonlinear kinematic joints is introduced to investigate the varying dynamic characteristics when worktable is subjected to combined load from six directions. The load-deformation relationship of each kinematic joint is dealt with a set of translational and angular spring elements. The nonlinear restoring force function of each joint involving coupling displacement is calculated. Based on the lumped mass method, the analytical 18-DOF dynamic equation is formulated by the analysis of force dependence between joints. Model verification tests are conducted. The worktable response exhibits the abundant and fascinating nonlinear phenomena arising in nonlinear joint and coupling effect. The nonlinear behavior behaves significant difference owing to the variations of excitation, platform position, screw length, preload and damping of joints. Thus, the model is promising for comprehension of machine dynamic behavior and for development of sophisticated control strategy.
\end{abstract}

Keywords: ball screw feed system; analytical model; coupling vibration; nonlinear dynamics; multi-body system

\section{Introduction}

With the ever-increasing development of modern manufacturing industry, demands for more precise and high-speed CNC (Computer Numerical Control) machine tools have been put forward. The quality and productivity of CNC machine tools are often dominated by the static and dynamic performances of feed drive system [1-4]. Further, vibration during processing is the essential factor that deteriorates surface finish and dimensional accuracy of components, such as the inferior product machining of thin-walled parts, integral impellers and high precision crankshafts [5-7]. An accurate dynamic model of feed drive system is essential for the prediction of structural vibration and the enhancement of operating behavior of CNC machine tools. The BSFS accompanied with rolling linear guideway platform is the popular unit to realize linear motion of CNC machine tools owing to good kinematic accuracy, smooth motions, low wear and long service lifetime without a stick-slip effect [8]. The approximately $60 \%$ of the total dynamic stiffness and approximately $90 \%$ of the total damping of the entire machine tool structure originates in the kinematic joints $[9,10]$, and then the complication and instability of vibration may be dramatically affected by the nonlinearity of joints among supported bearing, nut and carriage [11-13,5,14]. On the other hand, more accurate model considering structural nonlinearity is required by the virtual prototype and virtual machining [15]. Therefore, inclusion of the ball point contact of joints allows the present nonlinear vibration analysis to ensure controllable dynamic behaviors of BSFS.

Numerous scientific works have dealt with the analytical mechanical model of BSFS, with the aim of replicating the dynamical behavior as detailed as possible and improve the translational and positioning accuracy. Vicente et al. [16] developed a dynamic model for the first three resonant mode shapes analysis of BSFS. The axial-torsional coupling effect was investigated when screw shaft served as a continuous subsystem using a field function. However, the model was proposed in absence of experimental verification. Similarly, Dong and Tang [17] raised a mathematical model describing the continuous deformation of flexible screw using a field function with unknown bounds. Their model also contained time-varying parametric uncertainties and disturbances to investigate the tracking control for a high speed BSFS. Based on the Timoshenko beam assumption, Wu et al. [18] modeled the lead screw as a continuous body when the other

\footnotetext{
* Corresponding author

E-mail address: chyli@mail.neu.edu.cn (C. Li)
} 
parts of BSFS were treated as lumped mass units. The high-order modes of system and flexible feature of screw were characterized by the proposed hybrid model. Instead, Frey et al. [19] described the screw as a simple lumped mass and identified the ability of proposed model on expressing the most relevant characteristics of feed drives with respect to their application in machine tools. Sato [20] also reported a simple and physically intuitive 2-DOF model for expressing the first order mode of axial and rotational vibrations. It means that the axial stiffness of BSFS can be estimated as a serial connection of stiffness of the support bearing, screw shaft, and nut. Ansoategui and Campa [21] created a detailed 7-DOF lumped parameters dynamic model and compared with a classical 2-DOF model. The advantage of proposed model was that the 7-DOF model modal analysis could identify the weaker components of transmission during a redesign of the drive or transmission chain. Guo et al. [22] performed a novel idea for the hybrid dynamic model of BSFS through combining classical dynamics theory and data driving. Nguyen et al. [23] constructed a discrete dynamic model to investigate the interaction between preload, the displacement of working table and the axial natural frequency of screw-nut.

The nonlinearity of joints may result in substantial variation in natural frequencies and frequency responses of machine tool, which are rarely mentioned in antecedent literature. Considering the nonlinear stiffness of rolling guide joints owing to the point contact between ball and raceway, Wang et al. [24] developed a dynamic model of three-axis coupling machine tool to evaluate the axis coupling effect as well as position-dependent response of tool center point. On the basis of nonlinear contact characteristics of screw-nut and bearing joints, for vertical structure BSFS without counterweight [25], the slender BSFS [26] and BSFS with lead screw pre-stretching [27], the position-dependent transmission stiffness models of system were established respectively. Nevertheless, the above mentioned literatures pay little attention to nonlinear vibration behavior of BSFS which is necessary to evaluate the operational behavior of CNC machine tools under periodic cutting force treatment. $\mathrm{Li}$ et al. [28] and $\mathrm{Xu}$ et al. [29] developed a time-dependent dynamic model for clarifying the micro posture fluctuation mechanism of ball screw and carriage respectively. Due to the ball's point contact property, the inherent frequency multiplication and demultiplication components were captured when system was excited by the number variation of inserted loaded ball. Gu and Zhang [30] and Xu et al. [31] investigated the resonance state of BSFS using maximum amplitude-frequency curves. The result manifested the remarkable nonlinear phenomena, like super- and sub-harmonic resonance and jumping discontinuous behavior. Kong et al. [32] demonstrated the existence of unstable chaotic motion in linear guide through bifurcation diagram and maximum Lyapunov exponent. It is worth mentioning that these models ignored the coupling effect when the BSFS suffers from multi-directional loads, and were directed to the unidirectional vibration characteristics. Liu et al. [33] formulated a nonlinear dynamic model considering the assembly error of deflection angle caused by the misalignment between front and rear bearings. But the model discarded the guide platform which acts as the dominant carrying parts and the effect of torque was also not included. Although Wang et al. [13] derived the coupling relevance of linear guideways, screw-nut, screw shaft and supported bearings and studied the mechanical behavior of nonlinear kinematic joints for ball screw driven CNC axis, the yawing vibration behavior of worktable failed to be estimated and only the axial vibration response of screw-nut, shaft and bearings was highlighted. From the current research situation, most of the mechanical model for BSFS are limited to the axial dynamic characteristics, without involving the coupling effect of multi-directional loadings, which is incomplete for the accurate prediction of dynamic response of BSFS. This paper will try to establish a more comprehensive dynamic model to expand the research on structural dynamics of CNC machine tools.

In this paper, the combined load from vertical, lateral, feed, yawing, pitching and rolling directions is applied on the worktable of BSFS. In conventional machine tools, due to intensive FE optimization of machine structure in design, the structural parts are much stiffer than joints. The structural modal is at a relatively high frequency, and the predominant modes in the low-frequency and mid-frequency are entirely caused by limited joint stiffness. Thus, the dynamics of system is mainly dependent on the stiffness of joint rather than structures [24]. It is appropriate to model BSFS by lumped mass elements and a set of translational and angular spring elements for each kinematic joint. Aiming to describe the nonlinear contact of kinematic joint, the restoring force function of each supported part with respect to translation and angular displacements is calculated, involving two translations and three deflections of linear guideway platform, three translations and three deflections of screw-nut, three translations and two deflections of matched angular contact 
ball bearings, as well as two radial translations of supported deep groove ball bearing. Additionally, the screw shaft is treated as a simple lumped mass element and its three translations and two deflections (excluding the torsion deformation) are considered, furthermore, the position-dependent restoring force expression of lumped screw shaft unit is derived by the classical deformation formula of loaded beam. The approach aims at identifying the dominant effects of screw shaft, capable of expressing the most relevant position-dependent characteristics of BSFS. With the aid of lumped mass method, the analytical 18-DOF equation of motion with coupling displacement and nonlinear restoring force term is introduced. To the best of authors' knowledge, the proposed detailed model is not shared by any former author. Moreover, experiment is also arranged for the validations of calculation methodology and vibration response through a BSFS test bench. Nonlinear vibration analysis based on numerical solution is carried out according to amplitude-frequency curve, bifurcation diagram, 3-D frequency spectrum, velocity-displacement phase diagram, Poincaré section and frequency domain. Effect of external harmonic excitation, position-dependent, length of screw shaft, preload and damping of joint on dynamic response is discussed comparatively. The proposed model can be used to identify the mechanism of time domain vibration response, understand the posture variation, and develop precise numerical model algorithms to determine the vibration characteristics of BSFS.

\section{Dynamic model}

The most common BSFS for precision positioning consists of screw shaft, assembled to the machine pedestal by rotary bearings, which is driven by a servomotor through a flexible coupling, as shown in Fig. 1. The screw-nut joint is attached to worktable that is constrained to move axially on carriage and rail. For the fixed-supported installation of Fig. 1, the fixed end close to the motor is performed by a pair of angular contact ball bearings, and a deep groove ball bearing achieves the radial support of the other end. The right-hand Cartesian coordinate $X Y Z-O$ is defined and the origin $O$ lies on the symmetry center of top surface of worktable. The external loads $F_{x 0}, F_{y 0}, F_{z 0}$, $M_{x 0}, M_{y 0}, M_{z 0}$ from six directions are applied on the platform and the derived translation and angular displacements of platform center is $x, y, z, \theta_{x}, \theta_{y}, \theta_{z}$ corresponding to vertical, lateral, feed, yawing, pitching and rolling directions respectively.

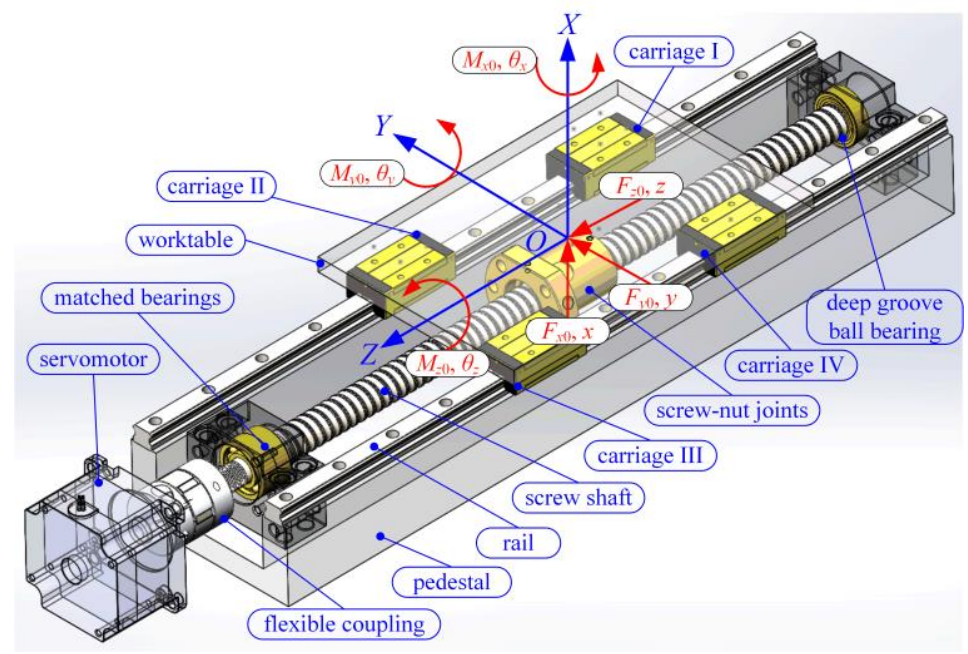

Fig. 1 Schematic diagram of ball screw driven $\mathrm{CNC}$ axis

\subsection{Equation of motion}

It is assumed that screw is suitably mounted in the servomechanism and non-concentric force produced by misalignments is discarded. The stiffness of servomotor and torsion deflection of flexible screw shaft are not considered. The rolling joints deformation is supposed to only occur in the contact area between ball and raceway. Fig. 2 shows the equivalent dynamic model of BSFS using the lumped mass method. The multi-body dynamical system is formulated by four lumped mass points and it refers to linear guideway platform which consists of four carriages, worktable and nut; flexible screw shaft; matched bearings and radial bearing. The rolling joints and flexible screw shaft are regarded as the spring-damper parts. The displacement vector is defined as $[x, y, z$, $\left.\theta_{x}, \theta_{y}, \theta_{z}, x_{s s}, y_{s s}, z_{s s}, \theta_{s s x}, \theta_{s s y}, x_{m b}, y_{m b}, z_{m b}, \theta_{m b x}, \theta_{m b y}, x_{d b}, y_{d b}\right]$, as shown in Fig. 2. Then, the 18-DOF differential equation of motion with time-vary restoring force term under harmonic excitation can be written as follows: 
for linear guideway platform:

$$
\left\{\begin{array}{l}
m_{p} x^{\prime \prime}+c_{l g x} x^{\prime}+F_{l g x}+c_{s n x}\left(x^{\prime}-x_{s s}^{\prime}\right)+F_{s n x}=F_{x 0} \sin \omega t \\
m_{p} y^{\prime \prime}+c_{l g y} y^{\prime}+F_{l g y}+c_{s n y}\left(y^{\prime}-y_{s s}^{\prime}\right)+F_{s n y}=F_{y 0} \sin \omega t \\
m_{p} z^{\prime \prime}+c_{s n z}\left(z^{\prime}-z_{s s}^{\prime}\right)+F_{s n z}=F_{z 0} \sin \omega t \\
I_{p x} \theta_{x}^{\prime \prime}+c_{l g \theta x} \theta_{x}^{\prime}+M_{l g x}+c_{s n \theta x}\left(\theta_{x}^{\prime}-\theta_{s s x}^{\prime}\right)+M_{s n x}=M_{x 0} \sin \omega t \\
I_{p y} \theta_{y}^{\prime \prime}+c_{l g \theta y} \theta_{y}^{\prime}+M_{l g y}+c_{s n \theta y}\left(\theta_{y}^{\prime}-\theta_{s s y}^{\prime}\right)+M_{s n y}=M_{y 0} \sin \omega t \\
I_{p z} \theta_{z}^{\prime \prime}+c_{l g \theta z} \theta_{z}^{\prime}+M_{l g z}+c_{s n \theta z} \theta_{z}^{\prime}+M_{s n z}=M_{z 0} \sin \omega t
\end{array}\right.
$$

where $m_{p}$ and $I_{p x}, I_{p y}, I_{p z}$ denote the mass and moment of inertia of platform (including table, carriage blocks, nut bracket and nut); ' and " are the first and second derivatives with respect to time $t ; \omega$ is excitation angular frequency; $x_{s s}, y_{s s}, z_{s s}, \theta_{s s x}, \theta_{s s y}$ represent translation and angular displacements of lumped screw shaft unit; $F_{l g x}, F_{l g y}, M_{l g x}, M_{l g y}, M_{l g z}$ are the synthetic restoring force and moment of four carriages; $F_{s n x}, F_{s n y}, F_{s n z}, M_{s n x}, M_{s n y}, M_{s n z}$ are restoring force and moment of nut joint; $c_{\lg x}, c_{\lg y}, c_{\lg \theta x}, c_{\lg \theta y}, c_{\lg \theta z}$ and $c_{s n x}, c_{s n y}, c_{s n z}, c_{s n \theta x}, c_{s n \theta y}, c_{s n \theta z}$ are the corresponding viscous damping coefficients. The approximated same damping for all ball contacts within the same joint is presumed. In reality, damping characteristic of elastic contact parts is nonlinear as well. In order to focus on the study of nonlinear elastic restoring force, the simplified constant proportional viscous damping coefficients are adopted for analyzing dynamic response owing to the fact of relatively small damping by analogy with rolling element bearing [34-37].

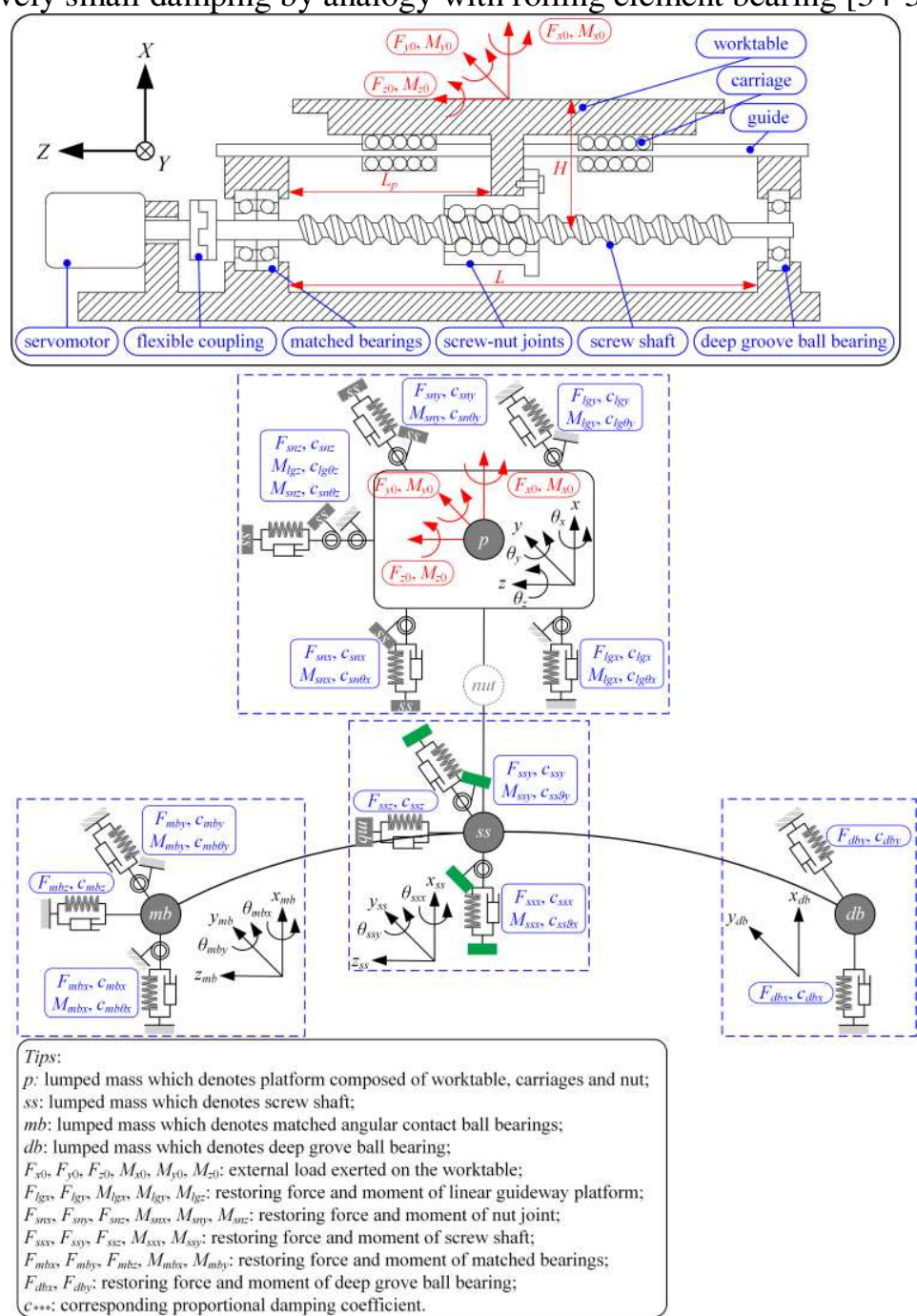

Fig. 2 Equivalent dynamic model of ball screw feed system

Because screw shaft is fixed on the base by bearings at both ends, the relationship between absolute displacement and deformation of lumped screw shaft unit should be clearly given before 
deriving its equation of motion. Fig. 3 describes the geometric relationship of screw displacement, deformation and bearing displacement. The translation and angular deformations of screw can be obtained:

at the $X O Z$ plane

$$
\left\{\begin{array}{l}
w_{s s x}=\left(x_{s s}-\left(\left(L-L_{p}\right) x_{m b}+L_{p} x_{d b}\right) / L\right) \frac{\cos \vartheta_{s s y}}{\cos \theta_{s s y}} \\
\vartheta_{s s y}=\theta_{s s y}-\psi_{1} \\
\psi_{1} \approx \tan \psi_{1}=\frac{x_{m b}-x_{d b}}{L-L_{p}}
\end{array}\right.
$$

at the $Y O Z$ plane

$$
\left\{\begin{array}{l}
w_{s s y}=\left(y_{s s}-\left(\left(L-L_{p}\right) y_{m b}+L_{p} y_{d b}\right) / L\right) \frac{\cos \vartheta_{s s x}}{\cos \theta_{s s x}} \\
\vartheta_{s s x}=\theta_{s s x}+\psi_{2} \\
\psi_{2} \approx \tan \psi_{2}=\frac{y_{m b}-y_{d b}}{L-L_{p}}
\end{array}\right.
$$

where $L$ is the length of screw shaft (interval between matched and radial bearings) and $L_{p}$ is the distance between platform and matched bearings; $x_{m b}, y_{m b}$ and $x_{d b}, y_{d b}$ are the radial displacements of matched and radial bearings, respectively; $\theta_{m b x}$ and $\theta_{m b y}$ are the angular displacements of matched bearing around vertical and lateral directions, respectively; $\psi_{1}$ and $\psi_{2}$ are the auxiliary parameter; $x_{s s}, y_{s s}$ and $\theta_{s s x}, \theta_{s s y}$ are the absolute translation and angular displacements of screw; $w_{s s x}$, $w_{s s y}$ and $\vartheta_{s s x}, \vartheta_{s s y}$ are the corresponding translation and angular deformations.
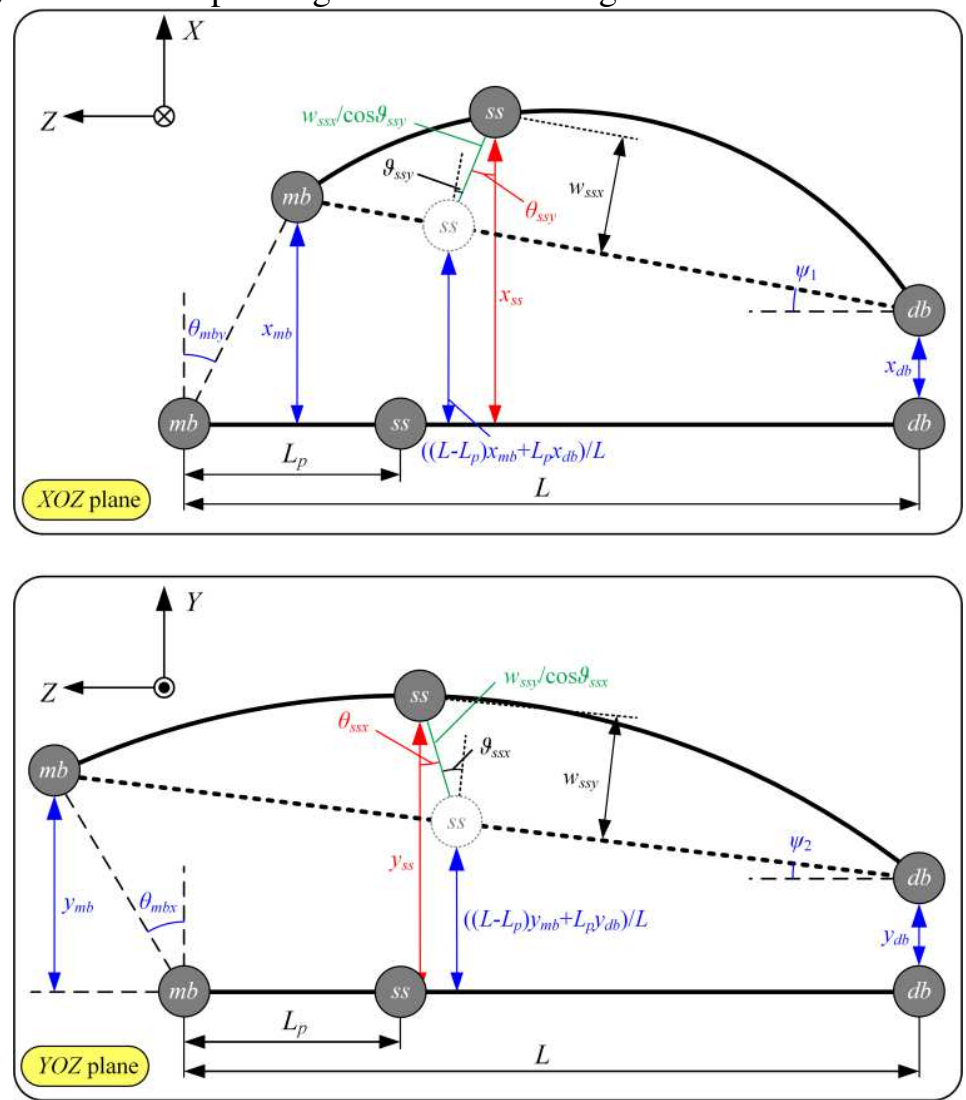

Fig. 3 The relationship between absolute displacement and deformation of lumped screw shaft unit Further, the equation of motion for screw can be formulated: 


$$
\left\{\begin{array}{l}
m_{s s} x_{s s}^{\prime \prime}+c_{s s x} w_{s s x}^{\prime}+F_{s s x}=F_{s n x}+c_{s n x}\left(x^{\prime}-x_{s s}^{\prime}\right) \\
m_{s s} y_{s s}^{\prime \prime}+c_{s s y} w_{s s y}^{\prime}+F_{s s y}=F_{s n y}+c_{s n y}\left(y^{\prime}-y_{s s}^{\prime}\right) \\
m_{s s} z_{s s}^{\prime \prime}+c_{s s z}\left(z_{s s}^{\prime}-z_{m b}^{\prime}\right)+F_{s s z}=F_{s n z}+c_{s n z}\left(z^{\prime}-z_{s s}^{\prime}\right) \\
I_{s s x} \theta_{s s x}^{\prime \prime}+c_{s s y x} \vartheta_{s s x}^{\prime}+M_{s s x}=M_{s n x}+c_{s n \theta x}\left(\theta_{x}^{\prime}-\theta_{s s x}^{\prime}\right) \\
I_{s s y} \theta_{s s y}^{\prime \prime}+c_{s s y y} \vartheta_{s s y}^{\prime}+M_{s s y}=M_{s n y}+c_{s n \theta y}\left(\theta_{y}^{\prime}-\theta_{s s y}^{\prime}\right)
\end{array}\right.
$$

where $m_{s s}$ and $I_{s s x}, I_{s s y}$ denote the mass and moment of inertia of screw; $F_{s s x}, F_{s s y}, F_{s s z}, M_{s s x}, M_{s s y}$ are the restoring force and moment of screw; $c_{s s x}, c_{s s y}, c_{s s z}, c_{s s} 9 x, c_{s s 9 y}$ are the corresponding damping coefficients; $z_{m b}$ is the displacement of matched bearing joints in feed direction.

For matched bearings, the equation of motion is expressed:

$$
\left\{\begin{array}{l}
m_{m b} x_{m b}^{\prime \prime}+c_{m b x} x_{m b}^{\prime}+F_{m b x}=F_{s s m b x}+c_{s s m b x} w_{s s x}^{\prime} \\
m_{m b} y_{m b}^{\prime \prime}+c_{m b y} y_{m b}^{\prime}+F_{m b y}=F_{s s m b y}+c_{s s m b y} w_{s s y}^{\prime} \\
m_{m b} z_{m b}^{\prime \prime}+c_{m b z} z_{m b}^{\prime}+F_{m b z}=F_{s s z}+c_{s s z}\left(z_{s s}^{\prime}-z_{m b}^{\prime}\right) \\
I_{m b x} \theta_{m b x}^{\prime \prime}+c_{m b \theta x} \theta_{m b x}^{\prime}+M_{m b x}=M_{s s m b x}+c_{s s m b \theta x} \vartheta_{s s x}^{\prime} \\
I_{m b y} \theta_{m b y}^{\prime \prime}+c_{m b \theta y} \theta_{m b y}^{\prime}+M_{m b y}=M_{s s m b y}+c_{s s m b \theta y} \vartheta_{s s y}^{\prime}
\end{array}\right.
$$

where $m_{m b}$ and $I_{m b x}, I_{m b y}$ denote the mass and moment of inertia of matched bearings (including inner and its shaft segment); $x_{m b}, y_{m b}, z_{m b}, \theta_{m b x}, \theta_{m b y}$ are the displacements of matched bearings joint in five vibration directions; $F_{m b x}, F_{m b y}, F_{m b z}, M_{m b x}, M_{m b y}$ are the restoring force and moment; $F_{s s m b x}, F_{s s m b y}, M_{s s m b x}, M_{\text {ssmby }}$ are the interaction force that exerts on matched bearings by screw, the specific expression is given in section $2.4 ; c_{m b x}, c_{m b y}, c_{m b z}, c_{m b \theta x}, c_{m b \theta y}, c_{s s m b x}, c_{s s m b y}, c_{s s m b \theta x}, c_{s s m b \theta y}$ are the corresponding damping coefficients.

For radial bearings,

$$
\left\{\begin{array}{l}
m_{d b} x_{d b}^{\prime \prime}+c_{d b x} x_{d b}^{\prime}+F_{d b x}=F_{s s d b x}+c_{s s d b x} w_{s s x}^{\prime} \\
m_{d b} y_{d b}^{\prime \prime}+c_{d b y} y_{d b}^{\prime}+F_{d b y}=F_{s s d b y}+c_{s s d b y} w_{s s y}^{\prime}
\end{array}\right.
$$

where $m_{d b}$ denotes the mass of deep groove ball bearing (including inner and its shaft segment); $x_{d b}, y_{d b}$ are the radial displacements; $F_{d b x}, F_{d b y}$ are restoring force; $F_{s s d b x}, F_{s s d b y}$ are the interaction force that exerts on radial bearing by screw, the specific expression is also given in section 2.4; $c_{d b x}, c_{d b y}, c_{s s d b x}, c_{s s d b y}$ are the corresponding damping coefficients.

\subsection{Calculation for elastic restoring force of linear guideway platform supported four carriages}

Fig. 4 shows the geometric information of platform and loaded ball arrangement between carriage and rail raceways. There are four rows of ball $(i=1,2,3,4)$ with face-to-face configuration inside one carriage. Each ball groove has a contact profile of circular arc forming a two-point contact mode. The oversized ball within groove enables a negative clearance $\delta_{l g 0}$ between raceway and ball that is necessary for high stiffness and stability of system. The groove curvature radii of carriage and rail are $r_{l g}$. The nominal diameter of ball is $d_{l g}$. The initial distance $O_{c i} O_{r i}$ between groove curvature centers of carriage and rail is $A_{l g 0}$ can be expressed:

$$
A_{l g 0}=2 r_{l g}-d_{l g}+\delta_{l g}
$$




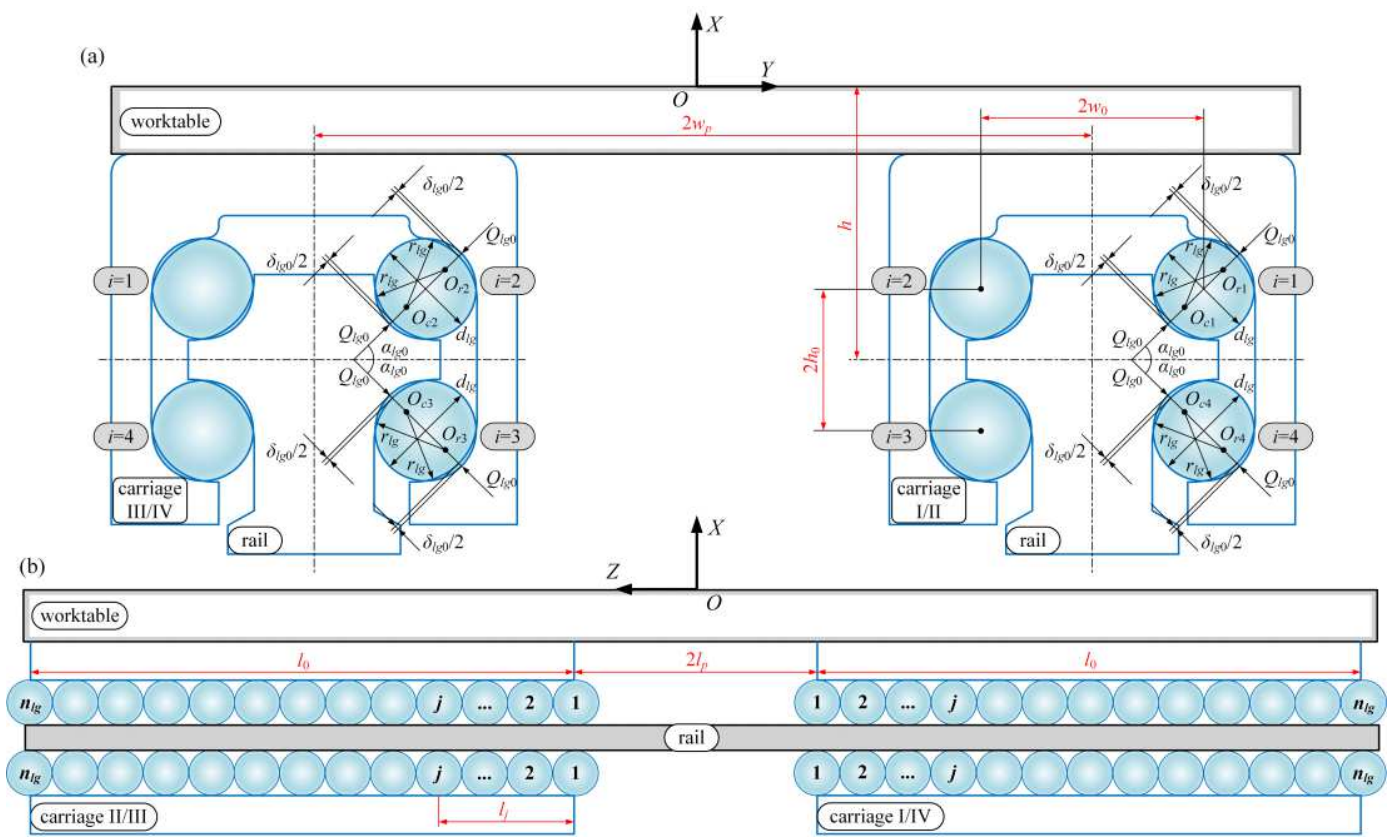

Fig. 4 Ball arrangement within carriage; (a) ball contact geometry with face-to-face configuration;

(b) ball location along feed direction

On the basis of Hertz contact theory, the normal preload $Q_{l g 0}$ of individual ball along contact angle $\alpha_{\lg 0}$ is $[38,39]:$

$$
Q_{l g 0}=K_{l g} \delta_{l g 0}^{3 / 2}
$$

where $K_{l g}$ is constant depending on material and geometric properties of contact area.

\subsubsection{The relationship between ball deformation and platform displacement}

As shown in Fig. 4(b), the identical loaded ball number $n_{l g}$ and ball arrangement in each row are assumed and loaded balls are distributed with equal intervals. A straight raceway of carriage with defect-free is supposed and the crowning design which is used to reduce ball passage vibration is not considered. According to the dimensions of carriage and platform, ball location along feed direction can be given:

$$
l_{j}=l_{p}+(j-1) \frac{l_{0}}{n_{l g}-1}
$$

where $j\left(j=1,2, \ldots n_{l g}\right)$ denotes the ball serial number; $2 l_{p}$ is the distance between carriage I and II or III and IV; $l_{0}$ is the length of carriage block. Then, the deformation of each ball can be obtained by the platform displacement and geometric relationship.

For carriage I, the ball deformation along $X, Y, Z$-axes can be calculated:

$$
\left\{\begin{array}{l}
\delta_{l g 1 \mid j x}=x-\left(w_{p}+w_{0}\right) \theta_{z}-l_{j} \theta_{y} \\
\delta_{l g 12 j x}=x-\left(w_{p}-w_{0}\right) \theta_{z}-l_{j} \theta_{y} \\
\delta_{l g 13 j x}=x-\left(w_{p}-w_{0}\right) \theta_{z}-l_{j} \theta_{y} \\
\delta_{l g 14 j x}=x-\left(w_{p}+w_{0}\right) \theta_{z}-l_{j} \theta_{y}
\end{array},\left\{\begin{array}{l}
\delta_{l g 11 j y}=y-\left(h-h_{0}\right) \theta_{z}+l_{j} \theta_{x} \\
\delta_{l g 12 j y}=y-\left(h-h_{0}\right) \theta_{z}+l_{j} \theta_{x} \\
\delta_{l g 13 j y}=y-\left(h+h_{0}\right) \theta_{z}+l_{j} \theta_{x} \\
\delta_{l g 14 j y}=y-\left(h+h_{0}\right) \theta_{z}+l_{j} \theta_{x}
\end{array},\left\{\begin{array}{l}
\delta_{l g 11 j z}=\left(h-h_{0}\right) \theta_{y}+\left(w_{p}+w_{0}\right) \theta_{x} \\
\delta_{l|l| 2 j z}=\left(h-h_{0}\right) \theta_{y}+\left(w_{p}-w_{0}\right) \theta_{x} \\
\delta_{l g 13 j z}=\left(h+h_{0}\right) \theta_{y}+\left(w_{p}-w_{0}\right) \theta_{x} \\
\delta_{l g 14 j z}=\left(h+h_{0}\right) \theta_{y}+\left(w_{p}+w_{0}\right) \theta_{x}
\end{array}\right.\right.\right.
$$

where $\delta_{l g I i j x}, \delta_{l g I i j y}$ and $\delta_{l g I i j z}$ represent the $j$ th ball elastic deformation of the $i$ th row along vertical, lateral and feed directions, respectively. As shown in Fig. 4(a), $2 w_{p}$ is the distance between carriage I and IV or II and III; $2 w_{0}$ is the distance between 1 and 2 rows of ball; $h$ is the distance between carriage center and top surface of platform and $h=0$ denotes that the $X$-axis passes through carriage center; $h_{0}$ is the distance between 2 and 3 rows of ball. Similarly, for carriage II, III and IV, the ball deformation along $X, Y, Z$-axes can be calculated:

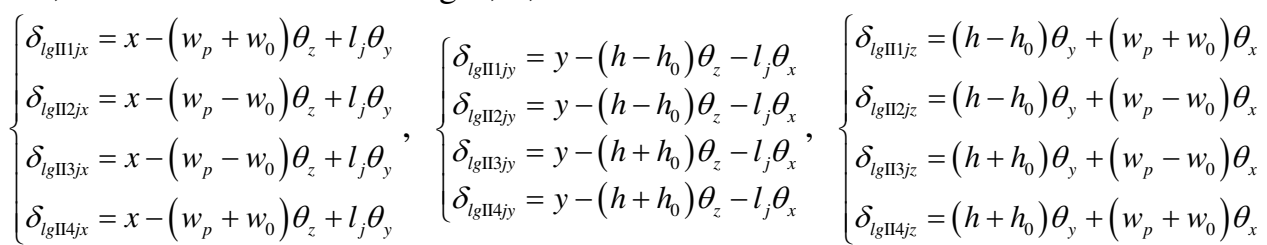




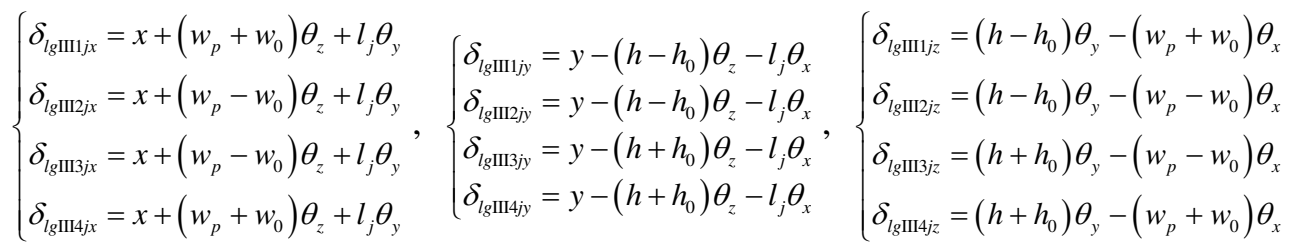

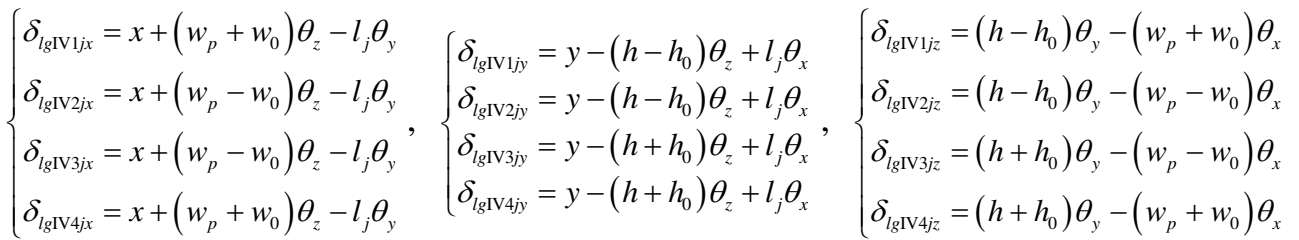

Then, the new curvature center distance $A_{\text {lgkij }}$ of $i=1,2,3,4$ row of the $j$ th ball under load can be obtained by:

for carriage I and II ( $\kappa=$ I or II),

$$
\left\{\begin{array}{l}
A_{l g \kappa 1 j}=\sqrt{\left(A_{l g 0} \sin \alpha_{l g 0}-\delta_{l g \kappa 1 j x}\right)^{2}+\left(A_{l g 0} \cos \alpha_{l g 0}-\delta_{l g \kappa 1 j y}\right)^{2}+\delta_{l g \kappa 1 j z}^{2}} \\
A_{l g \kappa 2 j}=\sqrt{\left(A_{l g 0} \sin \alpha_{l g 0}-\delta_{l g \kappa 2 j x}\right)^{2}+\left(A_{l g 0} \cos \alpha_{l g 0}+\delta_{l g \kappa 2 j y}\right)^{2}+\delta_{l g \kappa 2 j z}^{2}} \\
A_{l g \kappa 3 j}=\sqrt{\left(A_{l g 0} \sin \alpha_{l g 0}+\delta_{l g \kappa 3 j x}\right)^{2}+\left(A_{l g 0} \cos \alpha_{l g 0}+\delta_{l g \kappa 3 j y}\right)^{2}+\delta_{l g \kappa 3 j z}^{2}} \\
A_{l g \kappa 4 j}=\sqrt{\left(A_{l g 0} \sin \alpha_{l g 0}+\delta_{l g \kappa 4 j x}\right)^{2}+\left(A_{l g 0} \cos \alpha_{l g 0}-\delta_{l g \kappa 4 j y}\right)^{2}+\delta_{l g \kappa 4 j z}^{2}}
\end{array}\right.
$$

for carriage III and IV ( $\kappa=$ III or IV),

$$
\left\{\begin{array}{l}
A_{l g \kappa 1 j}=\sqrt{\left(A_{l g 0} \sin \alpha_{l g 0}-\delta_{l g \kappa 1 j x}\right)^{2}+\left(A_{l g 0} \cos \alpha_{l g 0}+\delta_{l g \kappa 1 j y}\right)^{2}+\delta_{l g \kappa 1 j z}^{2}} \\
A_{l g \kappa 2 j}=\sqrt{\left(A_{l g 0} \sin \alpha_{l g 0}-\delta_{l g \kappa 2 j x}\right)^{2}+\left(A_{l g 0} \cos \alpha_{l g 0}-\delta_{l g \kappa 2 j y}\right)^{2}+\delta_{l g \kappa 2 j z}^{2}} \\
A_{l g \kappa 3 j}=\sqrt{\left(A_{l g 0} \sin \alpha_{l g 0}+\delta_{l g \kappa 3 j x}\right)^{2}+\left(A_{l g 0} \cos \alpha_{l g 0}-\delta_{l g \kappa 3 j y}\right)^{2}+\delta_{l g \kappa 3 j z}^{2}} \\
A_{l g \kappa 4 j}=\sqrt{\left(A_{l g 0} \sin \alpha_{l g 0}+\delta_{l g \kappa 4 j x}\right)^{2}+\left(A_{l g 0} \cos \alpha_{l g 0}+\delta_{l g \kappa 4 j y}\right)^{2}+\delta_{l g \kappa 4 j z}^{2}}
\end{array}\right.
$$

The normal deformation $\delta_{l g k i j}$ of each ball under combined load can be described by:

$$
\delta_{l g \kappa i j}=A_{l g \kappa i j}-A_{l g 0}+\delta_{l g 0}
$$

According to Hertz contact theory, the normal load $Q_{l g k i j}$ of individual ball can be determined by:

$$
Q_{l g \kappa i j}=K_{l g} \delta_{l g \kappa i j}^{3 / 2}
$$

It is pointed out that when $\delta_{\text {lgkij }}<0$, the ball will lose contact with raceway surface and $\delta_{\text {lgkij }}$ is set to equal 0 . The spatial normal contact load $Q_{l g k i j}$ can be decomposed into $X, Y, Z$-axes directions:

$$
\left\{\begin{array}{l}
F_{l g \kappa i j x}=Q_{l g \kappa i j} \sin \alpha_{l g \kappa i j} \cos \beta_{l g \kappa i j} \\
F_{l g \kappa i j y}=Q_{l g \kappa i j} \cos \alpha_{l g \kappa i j} \cos \beta_{l g \kappa i j} \\
F_{l g \kappa i j z}=Q_{l g \kappa i j} \sin \beta_{l g \kappa i j}
\end{array}\right.
$$

where $F_{\text {lgkijx }}, F_{\text {lgkijy }}$ and $F_{\text {lgkijz }}$ denote the contact load of individual ball along vertical, lateral and feed directions, respectively; $\alpha_{\text {lgkij }}$ is the angle between $Y$-axis and the projection of new curvature center distance $A_{\text {lgkij }}$ on plane $X O Y ; \beta_{l g k i j}$ is the angle between new curvature center distance $A_{l g k i j}$ and plane $X O Y$; and they can be formulated by:

for carriage I and II ( $\kappa=\mathrm{I}$ or II), 


$$
\left\{\begin{array} { l } 
{ \operatorname { t a n } \alpha _ { l g \kappa 1 j } = \frac { A _ { l g 0 } \operatorname { s i n } \alpha _ { l g 0 } - \delta _ { l g \kappa 1 j x } } { A _ { l g 0 } \operatorname { c o s } \alpha _ { l g 0 } - \delta _ { l g \kappa 1 j y } } } \\
{ \operatorname { t a n } \alpha _ { l g \kappa 2 j } = \frac { A _ { l g 0 } \operatorname { s i n } \alpha _ { l g 0 } - \delta _ { l g \kappa 2 j x } } { A _ { l g 0 } \operatorname { c o s } \alpha _ { l g 0 } + \delta _ { l g \kappa 2 j y } } } \\
{ \operatorname { t a n } \alpha _ { l g \kappa 3 j } = \frac { A _ { l g 0 } \operatorname { s i n } \alpha _ { l g 0 } + \delta _ { l g \kappa 3 j x } } { A _ { l g 0 } \operatorname { c o s } \alpha _ { l g 0 } + \delta _ { l g \kappa 3 j y } } } \\
{ \operatorname { t a n } \alpha _ { l g \kappa 4 j } = \frac { A _ { l g 0 } \operatorname { s i n } \alpha _ { l g 0 } + \delta _ { l g \kappa 4 j x } } { A _ { l g 0 } \operatorname { c o s } \alpha _ { l g 0 } - \delta _ { l g \kappa 4 j y } } }
\end{array} \quad \left\{\begin{array}{l}
\tan \beta_{l g \kappa \kappa 2 j}=\frac{\delta_{l g \kappa 1 j z}}{\sqrt{\left(A_{l g 0} \sin \alpha_{l g 0}-\delta_{l g \kappa 1 j x}\right)^{2}+\left(A_{l g 0} \cos \alpha_{l g 0}-\delta_{l g \kappa 1 j y}\right)^{2}}} \\
\tan \beta_{l g \kappa 3 j}=\frac{\delta_{l g \kappa 2 j z}}{\sqrt{\left(A_{l g 0} \sin \alpha_{l g 0}-\delta_{l g \kappa 2 j x}\right)^{2}+\left(A_{l g 0} \cos \alpha_{l g 0}+\delta_{l g \kappa 2 j y}\right)^{2}}} \\
\tan \beta_{l g \kappa 4 j}=\frac{\delta_{l g \kappa 3 j z}}{\sqrt{\left(A_{l g 0} \sin \alpha_{l g 0}+\delta_{l g \kappa 3 j x}\right)^{2}+\left(A_{l g 0} \cos \alpha_{l g 0}+\delta_{l g \kappa \kappa 3 j y}\right)^{2}}}
\end{array}\right.\right.
$$

for carriage III and IV ( $\kappa=$ III or IV),

$$
\left\{\begin{array} { l } 
{ \operatorname { t a n } \alpha _ { l g \kappa 1 j } = \frac { A _ { l g 0 } \operatorname { s i n } \alpha _ { l g 0 } - \delta _ { l g \kappa 1 j x } } { A _ { l g 0 } \operatorname { c o s } \alpha _ { l g 0 } + \delta _ { l g \kappa 1 j y } } } \\
{ \operatorname { t a n } \alpha _ { l g \kappa 2 j } = \frac { A _ { l g 0 } \operatorname { s i n } \alpha _ { l g 0 } - \delta _ { l g \kappa 2 j x } } { A _ { l g 0 } \operatorname { c o s } \alpha _ { l g 0 } - \delta _ { l g \kappa 2 j y } } } \\
{ \operatorname { t a n } \alpha _ { l g \kappa 3 j } = \frac { A _ { l g 0 } \operatorname { s i n } \alpha _ { l g 0 } + \delta _ { l g \kappa 3 j x } } { A _ { l g 0 } \operatorname { c o s } \alpha _ { l g 0 } - \delta _ { l g \kappa 3 j y } } } \\
{ \operatorname { t a n } \alpha _ { l g \kappa 4 j } = \frac { A _ { l g 0 } \operatorname { s i n } \alpha _ { l g 0 } + \delta _ { l g \kappa 4 j x } } { A _ { l g 0 } \operatorname { c o s } \alpha _ { l g 0 } + \delta _ { l g \kappa 4 j y } } }
\end{array} \quad \left\{\begin{array}{l}
\tan \beta_{l g \kappa 2 j}=\frac{\delta_{l g \kappa 1 j z}}{\sqrt{\left(A_{l g 0} \sin \alpha_{l g 0}-\delta_{l g \kappa 1 j x}\right)^{2}+\left(A_{l g 0} \cos \alpha_{l g 0}+\delta_{l g \kappa 1 j y}\right)^{2}}} \\
\tan \beta_{l g \kappa 3 j}=\frac{\delta_{l g \kappa 2 j z}}{\sqrt{\left(A_{l g 0} \sin \alpha_{l g 0}-\delta_{l g \kappa 2 j x}\right)^{2}+\left(A_{l g 0} \cos \alpha_{l g 0}-\delta_{l g \kappa \kappa 3 j x}\right)^{2}+\left(A_{l g 0} \cos \alpha_{l g 0}-\delta_{l g \kappa 3 j y}\right)^{2}}} \\
\tan \beta_{l g \kappa 4 j}=\frac{\delta_{l g \kappa 4 j z}}{\sqrt{\left(A_{l g 0} \sin \alpha_{l g 0}+\delta_{l g \kappa 4 j x}\right)^{2}+\left(A_{l g 0} \cos \alpha_{l g 0}+\delta_{l g \kappa 4 j y}\right)^{2}}}
\end{array}\right.\right.
$$

\subsubsection{The synthetic restoring force function by four carriages}

In mathematical modeling, carriage and rail are connected with a series of spring elements with adequate stiffness. Based on a superposition of each ball load, the restoring force and moment of each carriage can be calculated by:

for carriage I,

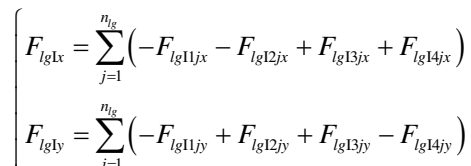

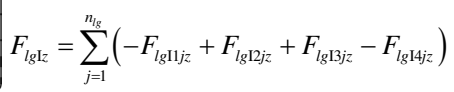

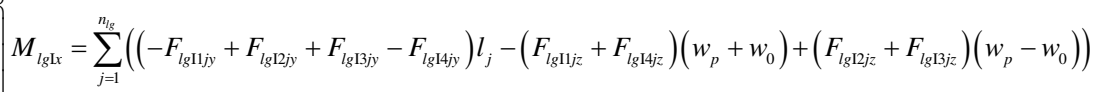

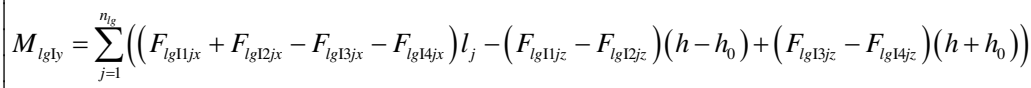

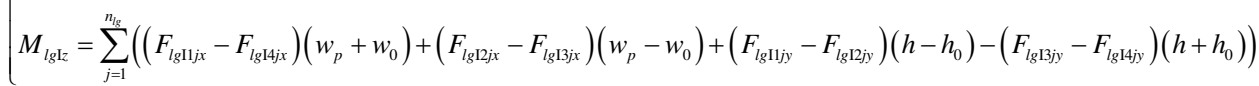

for carriage II, 


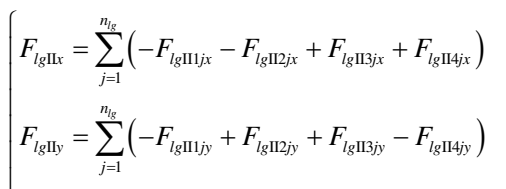

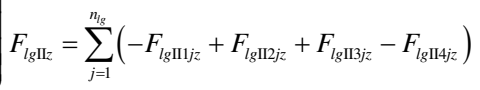

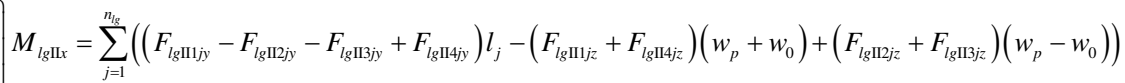

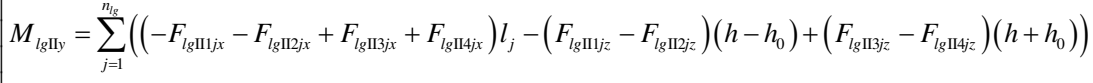

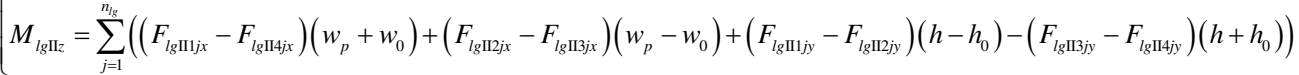

for carriage III,

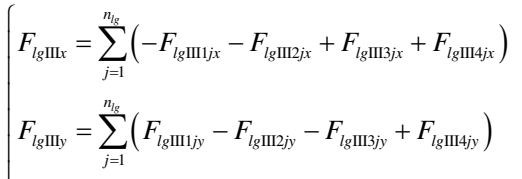

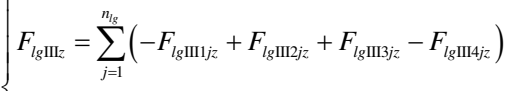

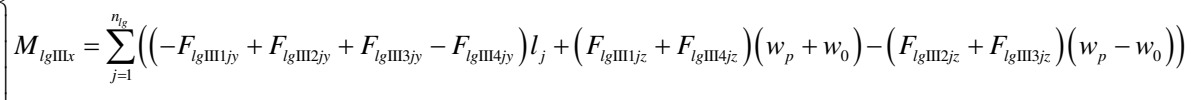

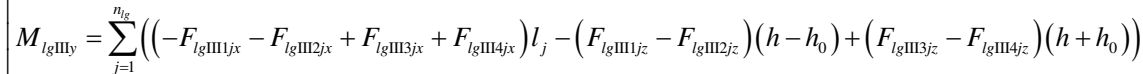

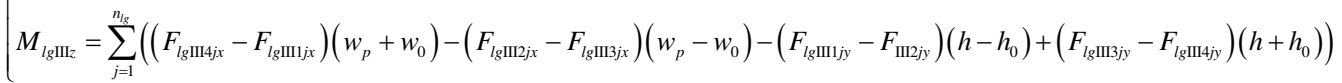

for carriage IV,

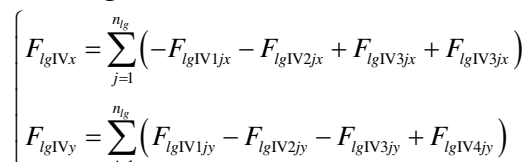

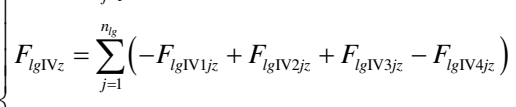

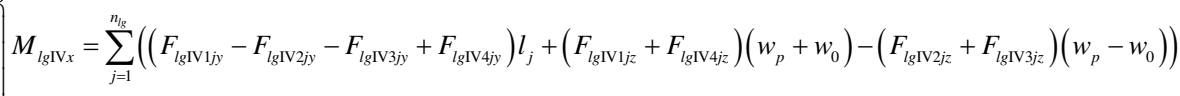

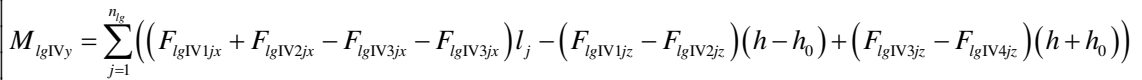

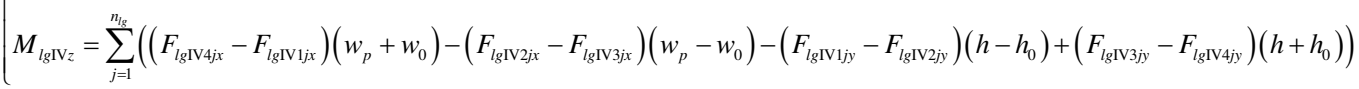

where $F_{l g k x}, F_{l g k y}, F_{l g k z}, M_{l g k x}, M_{l g k y}$ and $M_{\text {lgkz }}(\kappa=$ I,II,III,IV) denote the restoring force and moment of carriage $\kappa$. The synthetic force and moment can be obtained by a superposition of each carriage load. The expression can be given by: 


$$
\left\{\begin{array}{l}
F_{l g x}=\sum_{\kappa=\mathrm{I}}^{\mathrm{IV}} F_{l g \kappa x} \\
F_{l g y}=\sum_{\kappa=\mathrm{I}}^{\mathrm{IV}} F_{l g \kappa y} \\
F_{l g z}=\sum_{\kappa=\mathrm{I}}^{\mathrm{IV}} F_{l g \kappa z}=0 \\
M_{l g x}=\sum_{\kappa=\mathrm{I}}^{\mathrm{IV}} M_{l g \kappa x} \\
M_{l g y}=\sum_{\kappa=1}^{\mathrm{IV}} M_{l g \kappa y} \\
M_{l g z}=\sum_{\kappa=\mathrm{I}}^{\mathrm{IV}} M_{l g \kappa z}
\end{array}\right.
$$

\subsection{Calculation for elastic restoring force of nut joint}

The offset type preload screw-nut joint used in this paper has the benefit of a compact single-nut with high stiffness via small preload force for light to medium loading applications in contrast with the traditional double nut preload method. The preload of ball is adjusted by a $\sigma$ value offset on the center pitch $P_{s n}$ of nut on the two ball tracks as depicted in Fig. 5(a). The negative clearance $\delta_{s n 0}$ between raceway and ball can be achieved by the offset. The groove curvature radii of screw and nut are $r_{s n}$. The nominal diameter of ball is $d_{s n}$. The initial groove curvature center distance $O_{s l} O_{n l}$ or $O_{s r} O_{n r}$ of left or right side screw-nut is $A_{s n 0}$ and can be expressed:

$$
A_{s n 0}=2 r_{s n}-d_{s n}+\delta_{s n 0}
$$
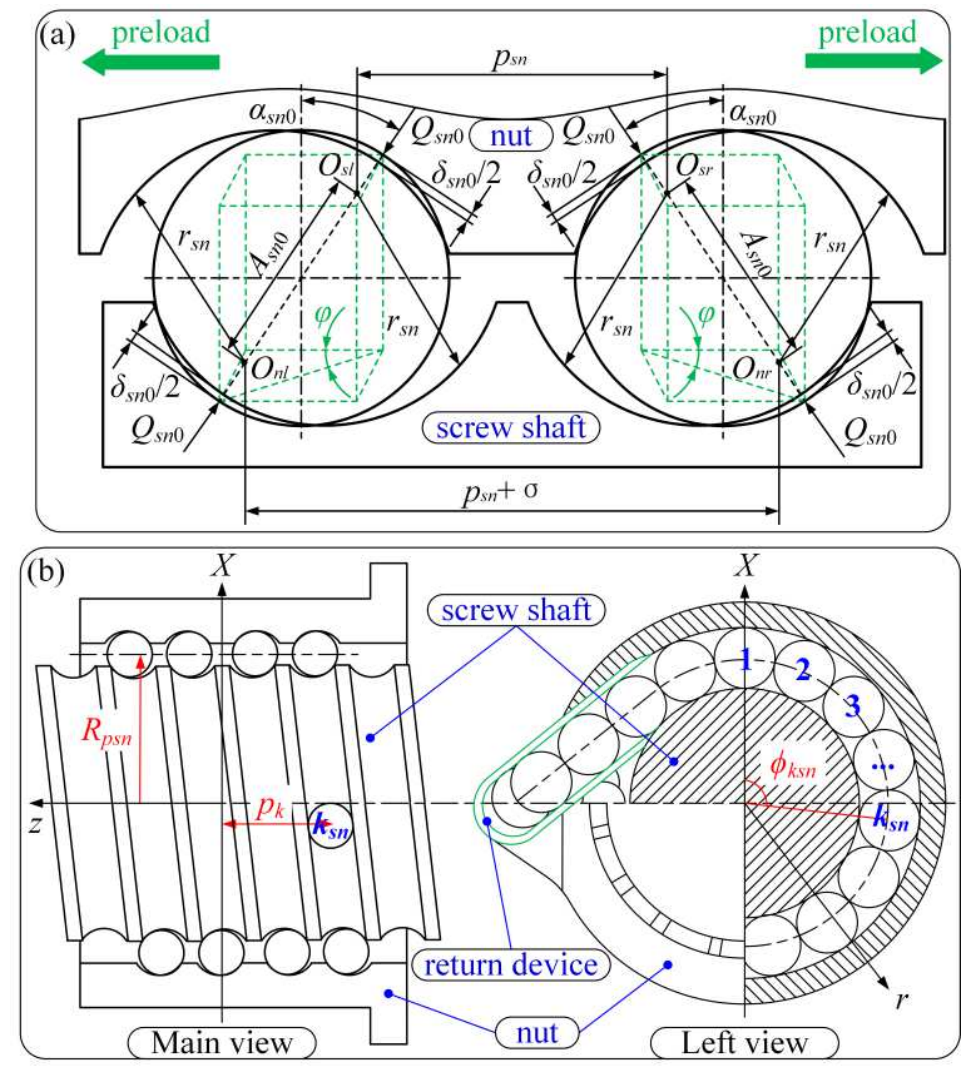

Fig. 5 Ball arrangement within screw-nut joint; (a) ball contact geometry; (b) ball location It is supposed that nut and screw in the nut length are rigid. On the basis of Hertz contact theory, the normal preload $Q_{s n 0}$ of individual ball along contact angle $\alpha_{s n 0}$ is [40]:

$$
Q_{s n 0}=K_{s n} \delta_{s n 0}^{3 / 2}
$$

where $K_{s n}$ is constant depending on material and geometric properties of contact area.

\subsubsection{The relationship between ball deformation within nut joint and platform displacement}


The loaded ball number $n_{s n}$ and ball arrangement in left and right-side nut are identical. The ideal screw loop raceway is assumed. As shown in Fig. 5(b), ball location along circumferential and axial direction can be given:

the radial position angle $\phi_{k s n}$ of the $k_{s n}$ th ball (measured from $+X$ axis):

$$
\phi_{k s n}=\frac{2 \pi n_{t}\left(k_{s n}-1\right)}{n_{s n}-1}
$$

where $n_{t}$ is ball's number of turns in one-side nut ( $n_{t}=2.5$ in this paper), $k_{s n}\left(k_{s n}=1,2, \ldots n_{s n}\right)$ denotes the ball serial number; the axial position $p_{k}$ of the $k_{s n}$ th ball:

$$
p_{k}=\frac{p_{s n} n_{t}\left(k_{s n}-1\right)}{n_{s n}-1}
$$

Then, the deformation of each ball under load can be obtained by platform and screw superimposed displacements and geometric relationship. For left and right-side nut, the $k_{s n}$ th ball deformation along radial( $r$-axis) and axial(z-axis) direction can be calculated by analogy with matched bearings [41]:

$$
\left\{\begin{array}{l}
\delta_{s n l z k}=\delta_{s n r z k}=\left(z-z_{s s}\right)+R_{p s n} \sin \phi_{k s n}\left(\theta_{x}-\theta_{s s x}\right)+\left(H-R_{p s n} \cos \phi_{k s n}\right)\left(\theta_{y}-\theta_{s s y}\right) \\
\delta_{s n l r k}=\left(\left(x-x_{s s}\right)-p_{k}\left(\theta_{y}-\theta_{s s y}\right)-R_{p s n} \sin \phi_{k s n} \theta_{z}\right) \cos \phi_{k s n}+\left(\left(y-y_{s s}\right)+p_{k}\left(\theta_{x}-\theta_{s s x}\right)-\left(H-R_{p s n} \cos \phi_{k s n}\right) \theta_{z}\right) \sin \phi_{k s n} \\
\delta_{s n r r k}=\left(\left(x-x_{s s}\right)+p_{k}\left(\theta_{y}-\theta_{s s y}\right)-R_{p s n} \sin \phi_{k s n} \theta_{z}\right) \cos \phi_{k s n}+\left(\left(y-y_{s s}\right)-p_{k}\left(\theta_{x}-\theta_{s s x}\right)-\left(H-R_{p s n} \cos \phi_{k s n}\right) \theta_{z}\right) \sin \phi_{k s n}
\end{array}\right.
$$

where $\delta_{\text {snlzk }}, \delta_{\text {snlrk }}$ and $\delta_{\text {snrzk }}, \delta_{\text {snrrk }}$ denote the $k_{s n}$ th ball deformation along $z$-axis and $r$-axis in the left and right nuts, respectively; the pitch circle radius is $R_{p s n} ; H$ represents vertical distance between origin located at the center of platform and screw axis, as shown in Fig. 2.

Then, the new curvature center distance of the $k_{s n}$ th ball under load is:

$$
\left\{\begin{array}{l}
A_{s n l k}=\sqrt{\left(A_{s n 0} \sin \alpha_{s n 0}-\delta_{s n l z k} \cos \varphi\right)^{2}+\left(A_{m b 0} \cos \alpha_{s n 0}+\delta_{s n l r k}\right)^{2}} \\
A_{s n n k}=\sqrt{\left(A_{s n 0} \sin \alpha_{s n 0}+\delta_{s n n z k} \cos \varphi\right)^{2}+\left(A_{s n 0} \cos \alpha_{s n 0}+\delta_{s n r k}\right)^{2}}
\end{array}\right.
$$

where $A_{\text {snlk }}$ and $A_{s n r k}$ denote the $k_{s n}$ th ball curvature center distance in the left and right nuts, respectively; the lead angle is $\varphi$ and can be expressed:

$$
\varphi=\tan ^{-1} \frac{p_{s n}}{2 \pi R_{p s n}}
$$

The normal deformation of each ball under combined load can be described by:

$$
\delta_{s n l k}=A_{s n l k}-A_{s n 0}+\delta_{s n 0}, \quad \delta_{s n r k}=A_{s n r k}-A_{s n 0}+\delta_{s n 0}
$$

where $\delta_{\text {snlk }}$ and $\delta_{\text {snrk }}$ denote the $k_{\text {sn }}$ th ball normal deformation in the left and right nuts. According to Hertz contact theory, the normal load of individual ball can be determined by:

$$
Q_{\text {snlk }}=K_{\text {sn }} \delta_{\text {snlk }}^{3 / 2}, Q_{\text {snrk }}=K_{s n} \delta_{\text {snrk }}^{3 / 2}
$$

where $Q_{\text {snlk }}$ and $Q_{s n r k}$ denote the $k_{s n}$ th ball normal load. It is pointed out that when $\delta_{\text {snlk }}$ or $\delta_{s n r k}<0$, the ball will lose contact with raceway surface and $\delta_{\text {snlk }}$ or $\delta_{\text {snrk }}$ is set to equal 0 . The spatial normal contact load $Q_{\text {snlk }}$ and $Q_{\text {snrk }}$ can be decomposed into $X, Y, Z$-axes directions:

$$
\left\{\begin{array}{l}
F_{\text {snlkk }}=Q_{\text {snlk }} \cos \alpha_{\text {snlk }} \cos \phi_{k s n} \\
F_{\text {snlyk }}=Q_{\text {snlk }} \cos \alpha_{\text {snlk }} \sin \phi_{k s n} \\
F_{\text {snlkk }}=Q_{\text {snlk }} \sin \alpha_{\text {snlk }} \cos \varphi
\end{array},\left\{\begin{array}{l}
F_{\text {snrxk }}=Q_{\text {snrk }} \cos \alpha_{\text {snrk }} \cos \phi_{k s n} \\
F_{\text {snryk }}=Q_{\text {snrk }} \cos \alpha_{\text {snrk }} \sin \phi_{k s n} \\
F_{\text {snrzk }}=Q_{\text {snrk }} \sin \alpha_{\text {snrk }} \cos \varphi
\end{array}\right.\right.
$$

where $F_{\text {snlxk }}, F_{\text {snlyk }}, F_{\text {snlzk }}$ and $F_{\text {snrxk }}, F_{\text {snryk }}, F_{\text {snrzk }}$ denote the load of $k_{s n}$ th ball in left and right nut along vertical, lateral and feed directions, respectively; $\alpha_{\text {snlk }}$ and $\alpha_{s n r k}$ are the new contact angles and it can be formulated by:

$$
\tan \alpha_{s n l k}=\frac{\delta_{s n l k k} \cos \varphi-A_{s n 0} \sin \alpha_{s n 0}}{A_{s n 0} \cos \alpha_{s n 0}+\delta_{s n l r k}}, \tan \alpha_{s n n k}=\frac{A_{s n 0} \sin \alpha_{s n 0}+\delta_{s n r k k} \cos \varphi}{A_{s n 0} \cos \alpha_{s n 0}+\delta_{s n r r k}}
$$

\subsubsection{The restoring force function of screw-nut joint}

Based on a superposition of each ball load, the restoring force and moment of screw-nut joint can be calculated by: 


$$
\left\{\begin{array}{l}
F_{s n x}=\sum_{k_{s n}=1}^{n_{s n}}\left(F_{s n l x k}+F_{s n x x k}\right) \\
F_{s n y}=\sum_{k_{s s n}=1}^{n_{s n}}\left(F_{s n l y k}+F_{s n n y k}\right) \\
F_{s n z}=\sum_{k_{s n}=1}^{n_{s n}}\left(F_{s n l z k}+F_{s n r z k}\right) \\
M_{s n x}=\sum_{k_{s n}=1}^{n_{s n}}\left(\left(F_{s n l z k}+F_{s n r z k}\right) R_{p s n} \sin \phi_{k s n}+\left(F_{s n l y k}-F_{s n r y k}\right) p_{k}\right) \\
M_{s n y}=\sum_{k_{s n}=1}^{n_{s n}}\left(\left(F_{s n r x k}-F_{s n l x k}\right) p_{k}+\left(F_{s n l z k}+F_{s n r z k}\right)\left(H-R_{p s n} \cos \phi_{k s n}\right)\right) \\
M_{s n z}=\sum_{k_{s n n}=1}^{n_{s n}}\left(-\left(F_{s n l x k}+F_{s n x x k}\right) R_{p s n} \sin \phi_{k s n}-\left(F_{s n l y k}+F_{s n r y k}\right)\left(H-R_{p s n} \cos \phi_{k s n}\right)\right)
\end{array}\right.
$$

\subsection{Calculation for position-dependent elastic restoring force of lumped screw} shaft unit

In this section, a simple approximation using lumped mass method is employed to model the screw shaft and this approach has been performed in Ref. $[11,24,19,23]$. The axial deformation $\delta_{s s z}$ of screw shaft may be expressed as:

$$
\delta_{s s z}=\frac{L_{p}}{E A} F_{s s z}
$$

where $E$ and $A$ are the elastic modulus and cross-sectional area of screw; the round rod with uniform cross section of diameter $d$ is used to model the screw. Then, the axial restoring force function of screw can be written:

$$
F_{s s z}=\frac{\pi E d^{2}}{4 L_{p}}\left(z_{s s}-z_{m b}\right)
$$

Fig. 6 shows the bending and deflection of screws under combined load and bearing constraints. The bending deformation at the radial bearing end is zero. At the XOZ plane, on the basis of deformation formula of classical loaded beam, the following equation can be obtained:

$$
\left\{\begin{array}{l}
w_{F_{s s x}}+w_{M_{s y}}+w_{F_{s s d b x}}=0 \\
w_{F_{s s x}}=\frac{F_{s s x} L_{p}^{2}}{6 E I}\left(3 L-L_{p}\right) \\
w_{M_{s y}}=-\frac{M_{s s y}}{2 E I}\left(2 L-L_{p}\right) \\
w_{F_{s s d b x}}=-\frac{F_{s s d b x} L^{3}}{3 E I} \\
I=\frac{\pi d^{4}}{64}
\end{array}\right.
$$

where $F_{s s d b x}$ is the supporting force of XOZ plane at radial bearing end; $w_{F s s x}, w_{M s s y}$ and $w_{F s s d b x}$ are deformation of radial bearing end along $X$-axis under $F_{s s x}, M_{s s y}$ and $F_{s s d b x}$, respectively; $I$ is equivalent area moment of inertia. The constraint can be derived:

$$
\left\{\begin{array}{l}
F_{s s d b x}=\frac{F_{s s x}}{2}\left(\frac{3 L_{p}^{2}}{L^{2}}-\frac{L_{p}^{3}}{L^{3}}\right)-\frac{3 M_{s s y}}{2}\left(\frac{2 L_{p}}{L^{2}}-\frac{L_{p}^{2}}{L^{3}}\right) \\
F_{s s m b x}=F_{s s x}-F_{s s d b x} \\
M_{s s m b y}=F_{s s x} L_{p}-F_{s s d b x} L-M_{s s y}
\end{array}\right.
$$

where $F_{s s m b x}$ and $M_{s s m b y}$ are the supporting force and moment of $X O Z$ plane at fixed matched bearings end. Further, the translational $w_{s s x}$ and angular $\vartheta_{s s y}$ deformation of lumped mass point of 
screw can be calculated:

$$
\left\{\begin{array}{l}
w_{s s x}=\frac{F_{s s x} L_{p}^{3}}{3 E I}-\frac{M_{s s y} L_{p}^{2}}{2 E I}-\frac{F_{s s d b x} L_{p}^{2}}{6 E I}\left(3 L-L_{p}\right) \\
\vartheta_{s s y}=\frac{F_{s s x} L_{p}^{2}}{2 E I}-\frac{M_{s s y} L_{p}}{E I}-\frac{F_{s s b b x} L_{p}}{2 E I}\left(2 L-L_{p}\right)
\end{array}\right.
$$

Then, the time-dependent restoring force function $F_{s s x}$ and $M_{s s y}$ can be expressed:

where

$$
\left\{\begin{array}{l}
F_{s s x}=\frac{P_{1} D-Q_{1} B}{A D-C B} \\
M_{s s y}=\frac{Q_{1} A-P_{1} C}{A D-C B}
\end{array}\right.
$$
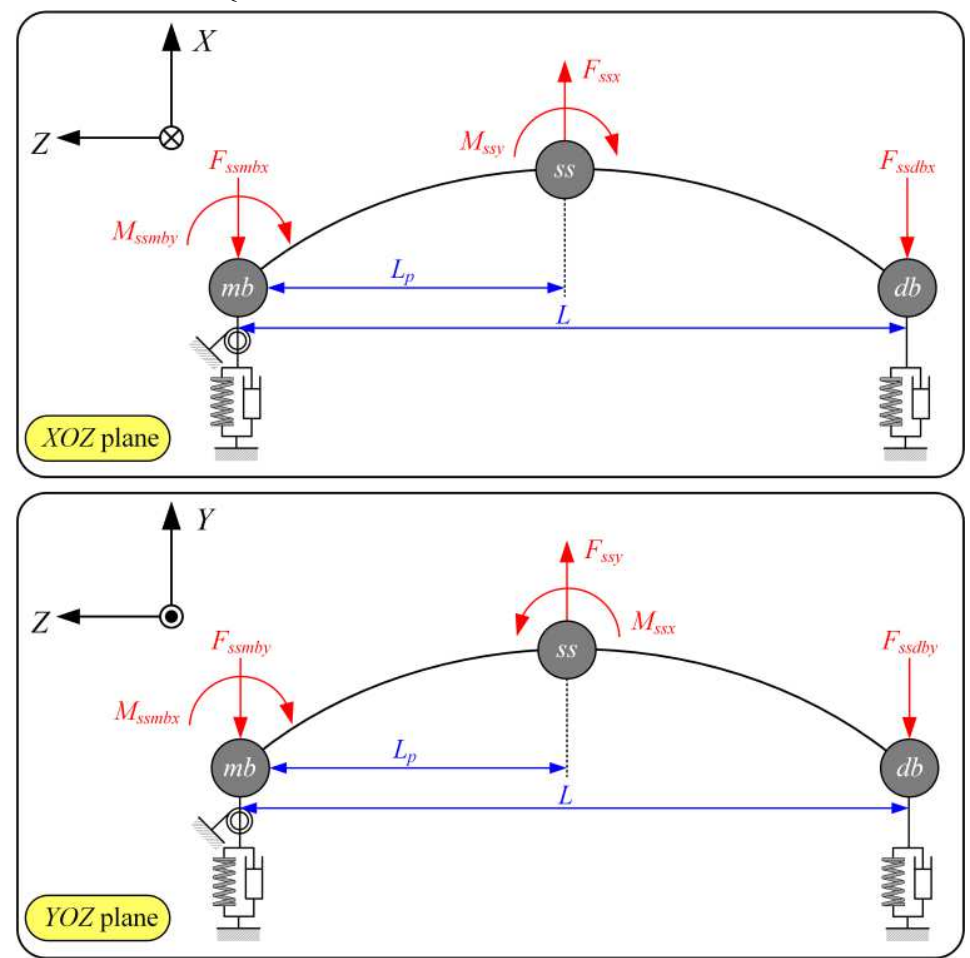

Fig. 6 Load analysis of screw shaft unit

Moreover, at the $Y O Z$ plane, the following equation can be obtained:

$$
\left\{\begin{array}{l}
w_{F_{s s y}}+w_{M_{s x x}}+w_{F_{s s d b y}}=0 \\
w_{F_{s y y}}=\frac{F_{s s y} L_{p}^{2}}{6 E I}\left(3 L-L_{p}\right) \\
w_{M_{s s x}}=\frac{M_{s s x}}{2 E I}\left(2 L-L_{p}\right) \\
w_{F_{s s d b y}}=-\frac{F_{s s d b y} L^{3}}{3 E I}
\end{array}\right.
$$


where $F_{\text {ssdby }}$ is the supporting force of $Y O Z$ plane at radial bearing end; $w_{F s s y}, w_{M s s x}$ and $w_{F s s d b y}$ are deformation of radial bearing end along $Y$-axis under $F_{s s y}, M_{s s x}$ and $F_{s s d b y}$, respectively. The constraint can be derived:

$$
\left\{\begin{array}{l}
F_{s s d b y}=\frac{F_{s s y}}{2}\left(\frac{3 L_{p}^{2}}{L^{2}}-\frac{L_{p}^{3}}{L^{3}}\right)+\frac{3 M_{s s x}}{2}\left(\frac{2 L_{p}}{L^{2}}-\frac{L_{p}^{2}}{L^{3}}\right) \\
F_{s s m b y}=F_{s s y}-F_{s s d b y} \\
M_{s s m b x}=F_{s s y} L_{p}-F_{s s d b y} L+M_{s s x}
\end{array}\right.
$$

where $F_{s s m b y}$ and $M_{s s m b x}$ are the supporting force and moment of $Y O Z$ plane at fixed matched bearings end. Further, the translational $w_{s s y}$ and angular $\vartheta_{s s x}$ deformation can be calculated:

$$
\left\{\begin{array}{l}
w_{s s y}=\frac{F_{s s y} L_{p}^{3}}{3 E I}+\frac{M_{s s x} L_{p}^{2}}{2 E I}-\frac{F_{s s d b y} L_{p}^{2}}{6 E I}\left(3 L-L_{p}\right) \\
\vartheta_{s s x}=\frac{F_{s s y} L_{p}^{2}}{2 E I}+\frac{M_{s s x} L_{p}}{E I}-\frac{F_{s s d b y} L_{p}}{2 E I}\left(2 L-L_{p}\right)
\end{array}\right.
$$

Then, the restoring force function $F_{s s y}$ and $M_{s s x}$ can be described:

where

$$
\left\{\begin{array}{l}
F_{s s y}=\frac{P_{2} D-Q_{2} B}{A D-C B} \\
M_{s s x}=\frac{P_{2} C-Q_{2} A}{A D-C B}
\end{array}\right.
$$

$$
\left\{\begin{array}{l}
P_{2}=12 E I L^{3} w_{s s y} \\
Q_{2}=4 E I L^{3} \vartheta_{s s x}
\end{array}\right.
$$

\subsection{Calculation for elastic restoring force of supported bearings}

\subsubsection{The elastic restoring force of matched angular contact ball bearings}

The matched bearings with back-to-back configuration achieve the support of fixed end of screw. The outer ring is fixed to the base. The preload with negative clearance $\delta_{m b 0}$ is applied to eliminate radial clearance, as depicted in Fig. 7(a). The groove curvature radii of inner and out rings are $r_{m b}$. The ball diameter is $d_{m b}$. The initial groove curvature center distance $O_{i l} O_{o l}$ or $O_{i r} O_{o r}$ of left or right bearing is $A_{m b 0}$ and can be expressed:

$$
A_{m b 0}=2 r_{m b}-d_{m b}+\delta_{m b 0}
$$




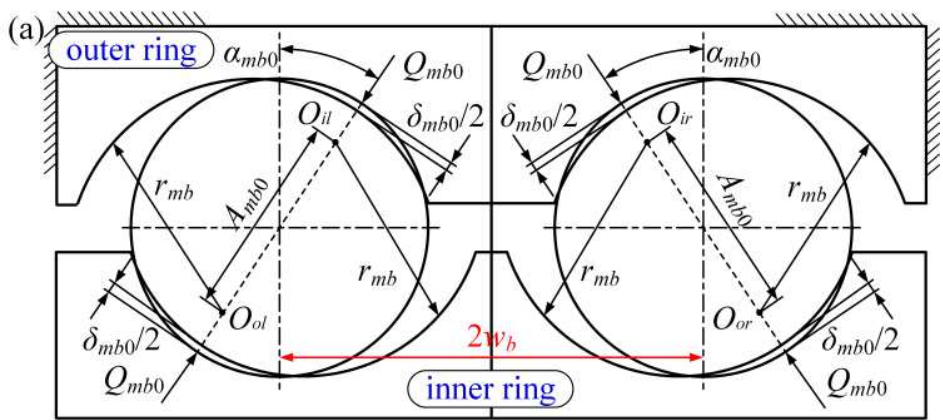

(b)

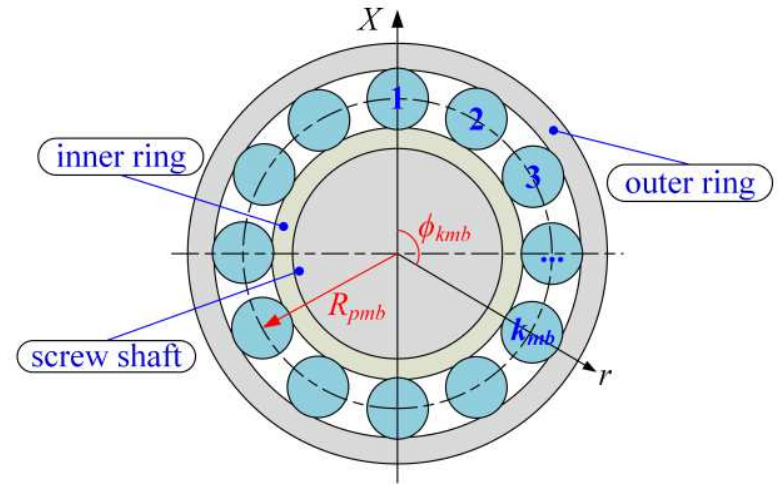

Fig. 7 Ball arrangement within matched bearing joint; (a) ball contact geometry; (b) ball location On the basis of Hertz contact theory, the normal preload $Q_{m b 0}$ of individual ball along contact angle $\alpha_{m b 0}$ is

$$
Q_{m b 0}=K_{m b} \delta_{m b 0}^{3 / 2}
$$

where $K_{m b}$ is constant depending on material and geometric properties of contact area.

As shown in Fig. 7(b), ball location along circumferential direction can be given:

$$
\phi_{k m b}=\frac{2 \pi\left(k_{m b}-1\right)}{n_{m b}}
$$

where $\phi_{k m b}$ is the radial position angle of the $k_{s n}$ th ball (measured from $+X$ axis); $n_{m b}$ is ball's number of one bearing, $k_{m b}\left(k_{m b}=1,2, \ldots n_{m b}\right)$ denotes the ball serial number. Then, the deformation of each ball under load can be obtained by inner ring displacement $\left(x_{m b}, y_{m b}, z_{m b}, \theta_{m b x}, \theta_{m b y}\right)$ and geometric relationship. For left and right bearing, the $k_{m b}$ th ball deformation along radial $(r$-axis) and axial direction can be calculated:

$$
\left\{\begin{array}{l}
\delta_{m b l z k}=\delta_{m b r z k}=z_{m b}+R_{p m b} \sin \phi_{k m b} \theta_{m b x}-R_{p m b} \cos \phi_{k m b} \theta_{m b y} \\
\delta_{m b l r k}=\left(x_{m b}-w_{b} \theta_{m b y}\right) \cos \phi_{k m b}+\left(y_{m b}+w_{b} \theta_{m b x}\right) \sin \phi_{k m b} \\
\delta_{m b r r k}=\left(x_{m b}+w_{b} \theta_{m b y}\right) \cos \phi_{k m b}+\left(y_{m b}-w_{b} \theta_{m b x}\right) \sin \phi_{k m b}
\end{array}\right.
$$

where $\delta_{m b l z k}, \delta_{m b l r k}$ and $\delta_{m b r z k}, \delta_{m b r r k}$ denote the $k_{m b}$ th ball deformation along $z$-axis and $r$-axis in the left and right bearings, respectively; the pitch circle radius is $R_{p m b} ; 2 w_{b}$ represents width of bearing. Then, the new curvature center distance of the $k_{m b}$ th ball under load is:

$$
\left\{\begin{array}{l}
A_{m b l k}=\sqrt{\left(A_{m b 0} \sin \alpha_{m b 0}-\delta_{m b l z k}\right)^{2}+\left(A_{m b 0} \cos \alpha_{m b 0}+\delta_{m b l r k}\right)^{2}} \\
A_{m b r k}=\sqrt{\left(A_{m b 0} \sin \alpha_{m b 0}+\delta_{m b r z k}\right)^{2}+\left(A_{m b 0} \cos \alpha_{m b 0}+\delta_{m b r r k}\right)^{2}}
\end{array}\right.
$$

where $A_{m b l k}$ and $A_{m b r k}$ denote the $k_{m b}$ th ball curvature center distance in the left and right bearings, respectively. The normal deformation under combined load can be described by:

$$
\delta_{m b l k}=A_{m b l k}-A_{m b 0}+\delta_{m b 0}, \quad \delta_{m b r k}=A_{m b r k}-A_{m b 0}+\delta_{m b 0}
$$

where $\delta_{m b l k}$ and $\delta_{m b r k}$ denote the $k_{m b}$ th ball normal deformation in the left and right bearings, respectively. According to Hertz contact theory, the normal load of individual ball can be determined by:

$$
Q_{m b l k}=K_{m b} \delta_{m b l k}^{3 / 2}, \quad Q_{m b r k}=K_{m b} \delta_{m b r k}^{3 / 2}
$$

It is pointed out that when $\delta_{m b l k}$ or $\delta_{m b r k}<0$, the ball will lose contact with raceway surface and $\delta_{m b l k}$ or $\delta_{m b r k}$ is set to equal 0 . The spatial normal load $Q_{m b l k}$ and $Q_{m b r k}$ can be decomposed into $X, Y$, 
Z-axes directions:

$$
\left\{\begin{array}{l}
F_{m b l x k}=Q_{m b l k} \cos \alpha_{m b l k} \cos \phi_{k m b} \\
F_{m b l y k}=Q_{m b l k} \cos \alpha_{m b l k} \sin \phi_{k m b} \\
F_{m b l z k}=Q_{m b l k} \sin \alpha_{m b l k}
\end{array},\left\{\begin{array}{l}
F_{m b r x k}=Q_{m b r k} \cos \alpha_{m b r k} \cos \phi_{k m b} \\
F_{m b r y k}=Q_{m b r k} \cos \alpha_{m b r k} \sin \phi_{k m b} \\
F_{m b r z k}=Q_{m b r k} \sin \alpha_{m b r k}
\end{array}\right.\right.
$$

where $F_{m b l x k}, F_{m b l y k}, F_{m b l z k}$ and $F_{m b r x k}, F_{m b r y k}, F_{m b r z k}$ denote the contact load of $k_{m b}$ th ball in the left and right bearing along vertical, lateral and feed directions, respectively; $\alpha_{m b l k}$ and $\alpha_{m b r k}$ are the new contact angles and it can be formulated by:

$$
\tan \alpha_{m b l k}=\frac{\delta_{m b l z k}-A_{m b 0} \sin \alpha_{m b 0}}{A_{m b 0} \cos \alpha_{m b 0}+\delta_{m b l r k}}, \tan \alpha_{m b r k}=\frac{A_{m b 0} \sin \alpha_{m b 0}+\delta_{m b r z k}}{A_{m b 0} \cos \alpha_{m b 0}+\delta_{m b r r k}}
$$

Based on a superposition of each ball load, the restoring force and moment of matched bearings joint can be calculated by:

$$
\left\{\begin{array}{l}
F_{m b x}=\sum_{k_{m b}=1}^{n_{m b}}\left(F_{m b l x k}+F_{m b r x k}\right) \\
F_{m b y}=\sum_{k_{m b}=1}^{n_{m b}}\left(F_{m b l y k}+F_{m b r y k}\right) \\
F_{m b z}=\sum_{k_{m b}=1}^{n_{m b}}\left(F_{m b l z k}+F_{m b r z k}\right) \\
M_{m b x}=\sum_{k_{m b}=1}^{n_{m b}}\left(\left(F_{m b l k k}+F_{m b r z k}\right) R_{p m b} \sin \phi_{k m b}+\left(F_{m b l y k}-F_{m b r y k}\right) w_{b}\right) \\
M_{m b y}=\sum_{k_{m b}=1}^{n_{m b}}\left(\left(F_{m b r x k}-F_{m b l x k}\right) w_{b}-\left(F_{m b l z k}+F_{m b r k k}\right) R_{p m b} \cos \phi_{k m b}\right)
\end{array}\right.
$$

\subsubsection{The elastic restoring force of deep groove ball bearing}

For the deep groove ball bearing at the free end of screw shaft, deflection and axial deformations of ball are ignored. Similar to matched bearings, the ball location along circumferential direction can be given:

$$
\phi_{k d b}=\frac{2 \pi\left(k_{d b}-1\right)}{n_{d b}}
$$

where $\phi_{k d b}$ is the radial position angle of the $k_{d b}$ th ball; $n_{d b}$ is ball's number of one bearing, $k_{d b}$ $\left(k_{d b}=1,2, \ldots n_{d b}\right)$ denotes the ball serial number. Then, the deformation of each ball under load can be obtained by inner ring displacement $\left(x_{d b}, y_{d b}\right)$ and geometric relationship. The $k_{d b}$ th ball deformation along radial direction can be calculated:

$$
\delta_{d b r k}=x_{d b} \cos \phi_{k d b}+y_{d b} \sin \phi_{k d b}-\gamma
$$

where $\delta_{d b r k}$ denotes the $k_{d b}$ th ball deformation along radial direction; $\gamma$ is radial clearance. On the basis of Hertz contact theory, the normal load $Q_{d b r k}$ of the $k_{d b}$ th ball is:

$$
Q_{d b r k}=K_{d b} \delta_{d b r k}^{3 / 2}
$$

where $K_{d b}$ is constant depending on material and geometric properties of contact area. When $\delta_{d b r k}$ $<0$, the ball will lose contact with raceway surface and $\delta_{d b r k}$ is set to equal $0 . Q_{d b r k}$ can be decomposed into $X, Y$-axes directions:

$$
F_{d b x k}=Q_{d b r k} \cos \phi_{k d b}, \quad F_{d b y k}=Q_{d b r k} \sin \phi_{k d b}
$$

where $F_{d b x k}$ and $F_{d b y k}$ denote the contact load of individual ball along vertical and lateral directions, respectively. Based on a superposition of each ball load, the restoring force of deep groove ball bearing joint can be calculated by:

$$
F_{d b x}=\sum_{k_{d b}=1}^{n_{d b}} F_{d b x k}, \quad F_{d b y}=\sum_{k_{d b}=1}^{n_{d b}} F_{d b y k}
$$

\section{Result and discussion}

\subsection{Model verification}

The model parameters of studied ball screw feed system are given in Table 1 . The above differential equations of proposed model with 18-DOF are solved by the fourth-order Runge-Kutta 
algorithm. The initial displacement and velocity of mechanical system are chosen as zero. The error for numerical approximation caused by time discretization matters the convergence of method. Thus, the termination criterion is specified to an error tolerance of less than $10^{-7}$ to satisfy the requirements for convergent solution. A linear viscous damping is adopted in this study, and the damping of bearing is about $0.25-2.5 \times 10^{5}$ times of linearized stiffness of bearing [42], and the damping coefficient is determined within this range.

Table 1 Specification of system

\begin{tabular}{|c|c|}
\hline Parameter & Value \\
\hline Carriage size (length, width, height) $(\mathrm{mm})$ & $80.4,100,40.5$ \\
\hline Rail size (width, height) (mm) & 34,29 \\
\hline$w_{0}, h_{0}, l_{0}(\mathrm{~mm})$ & $17,6.5,80.4$ \\
\hline$l_{p}, w_{p}, h(\mathrm{~mm})$ & $105,175,44$ \\
\hline$H(\mathrm{~mm})$ & 96 \\
\hline Distance between support bearings $L(\mathrm{~mm})$ & 800 \\
\hline Thickness of platform (mm) & 20 \\
\hline Mass of carriage (kg) & 1.6 \\
\hline Mass of nut (kg) & 8.8 \\
\hline Mass of bearing $(\mathrm{kg})$ & 0.2 \\
\hline Mass of screw shaft $(\mathrm{kg} / \mathrm{m})$ & 9.09 \\
\hline Lumped platform mass $m_{p}(\mathrm{~kg})$ & 33 \\
\hline Lumped bearing mass $m_{m b}, m_{d b}(\mathrm{~kg})$ & $0.576,0.288$ \\
\hline Inertia of platform $I_{p x}, I_{p y}$ and $I_{p z}\left(\mathrm{~kg} \cdot \mathrm{m}^{2}\right)$ & $0.76661,0.31112,0.50571$ \\
\hline Inertia of shaft $I_{s s x}$ or $I_{s s y}\left(\mathrm{~kg} \cdot \mathrm{m}^{2}\right)$ & 0.38851 \\
\hline Inertia of bearing $I_{m b x}$ or $I_{m b y}\left(\mathrm{~kg} \cdot \mathrm{m}^{2}\right)$ & 0.00013 \\
\hline Ball diameter within bearing $d_{m b}$ and $d_{d b}(\mathrm{~mm})$ & 8.80 \\
\hline Ball number of each row within carriage $n_{l g}$ & 13 \\
\hline Loaded ball number within one-side nut $n_{s n}$ & 23 \\
\hline Ball number of one bearing $n_{m b}$ or $n_{d b}$ & 13 \\
\hline Dimensionless raceway radius ratio $f_{0}$ & 0.52 \\
\hline Initial contact angle $\alpha_{l g 0}, \alpha_{s n 0}$ and $\alpha_{m b 0}$ (degree) & $45,45,40$ \\
\hline Pitch diameter of screw shaft $d_{p s n}(\mathrm{~mm})$ & 42 \\
\hline Pitch diameter of bearing $d_{p m b}(\mathrm{~mm})$ & 46 \\
\hline Diameter of screw shaft $d(\mathrm{~mm})$ & 38.5 \\
\hline Width of bearing $2 w_{b}(\mathrm{~mm})$ & 16 \\
\hline Lead $p_{s n}(\mathrm{~mm})$ & 16 \\
\hline Preload of carriage, nut and bearing $(\mathrm{N})$ & $623,1690,870$ \\
\hline Elastic modulus $E(\mathrm{GPa})$ & 206 \\
\hline $\begin{array}{c}\text { Damping coefficients of carriage, nut and } \\
\text { bearing }(\mathrm{N} \cdot \mathrm{s} / \mathrm{m})\end{array}$ & $2.7 \times 10^{4}, 0.8 \times 10^{4}, 0.4 \times 10^{4}$ \\
\hline Hertz coefficient $K_{l g}\left(\mathrm{~N} / \mathrm{m}^{3 / 2}\right)$ & $9.14849 \times 10^{9}$ \\
\hline Hertz coefficient $K_{s n}\left(\mathrm{~N} / \mathrm{m}^{3 / 2}\right)$ & $8.07171 \times 10^{9}$ \\
\hline Hertz coefficient $K_{m b}$ and $K_{d b}\left(\mathrm{~N} / \mathrm{m}^{3 / 2}\right)$ & $8.53657 \times 10^{9}$ \\
\hline
\end{tabular}

Fig. 8(a) shows the excitation test arrangement. The axial excitation position is selected for the response validation due to the coupled pitching and feed vibrations. The harmonic excitation signal is generated by shaker and applied on the table with aid of sting and force sensor. The axial vibration responses of piezoelectric accelerometer and force data are picked up simultaneously at a sample frequency of $20 \mathrm{kHz}$. The excitation with amplitude $50 \mathrm{~N}, 100 \mathrm{~N}, 200 \mathrm{~N}$ and various frequency of $60-100 \mathrm{~Hz}$ with the step of $5 \mathrm{~Hz}$ is produced. The 3-D frequency spectrum is used to compare the difference between experimental and theoretical results, as shown in Fig. 9. The fundamental frequency $f$ and its multiples $2 f, 3 f$ are detected, and measured and predicted values are very close in amplitude. The examples of $100 \mathrm{~N}, 80 \mathrm{~Hz}$ and $200 \mathrm{~N}, 100 \mathrm{~Hz}$ in Fig. 10 are selected for the detailed observation. The predicted amplitude of primary frequency $f$ shows a high conformity with measured one while there are some distinction between amplitudes of $2 f$ and $3 f$. Some differences exist between the vibration amplitudes of the predicted and measured results, but the change law of predicted vibration is considerably similar to that of measured one when various excitation is applied. 

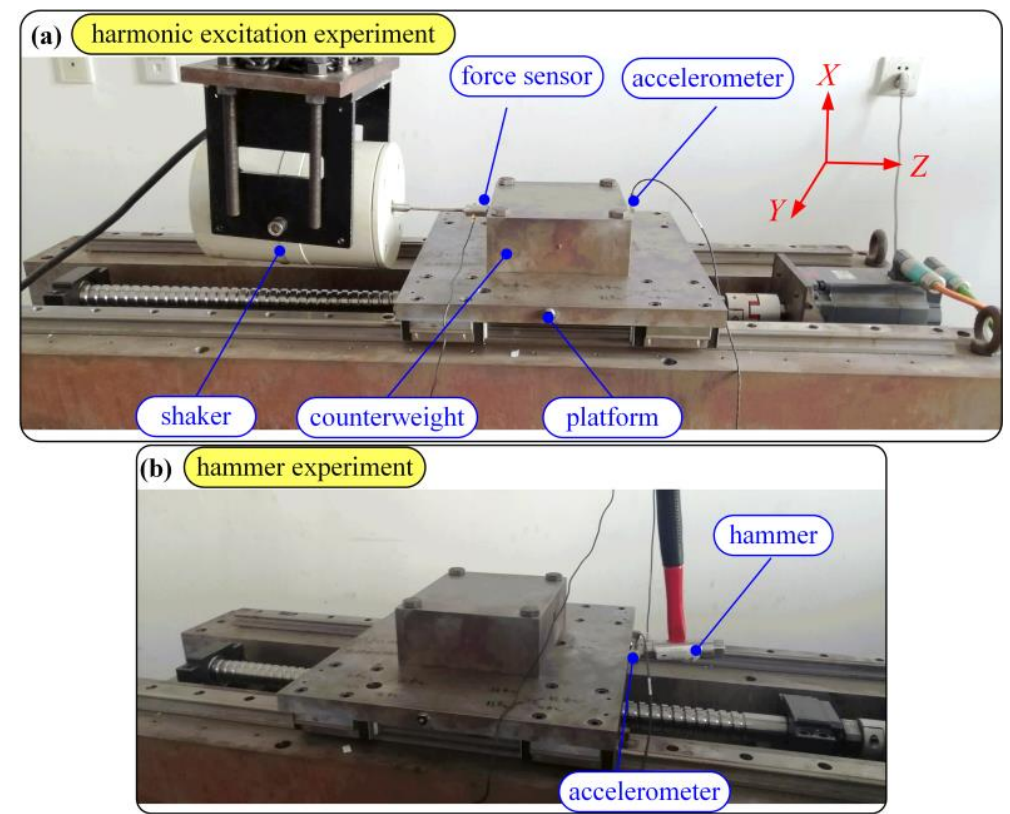

Fig. 8 Dynamics measurement; (a) experimental setup for harmonic excitation; (b) impact testing instrument setup

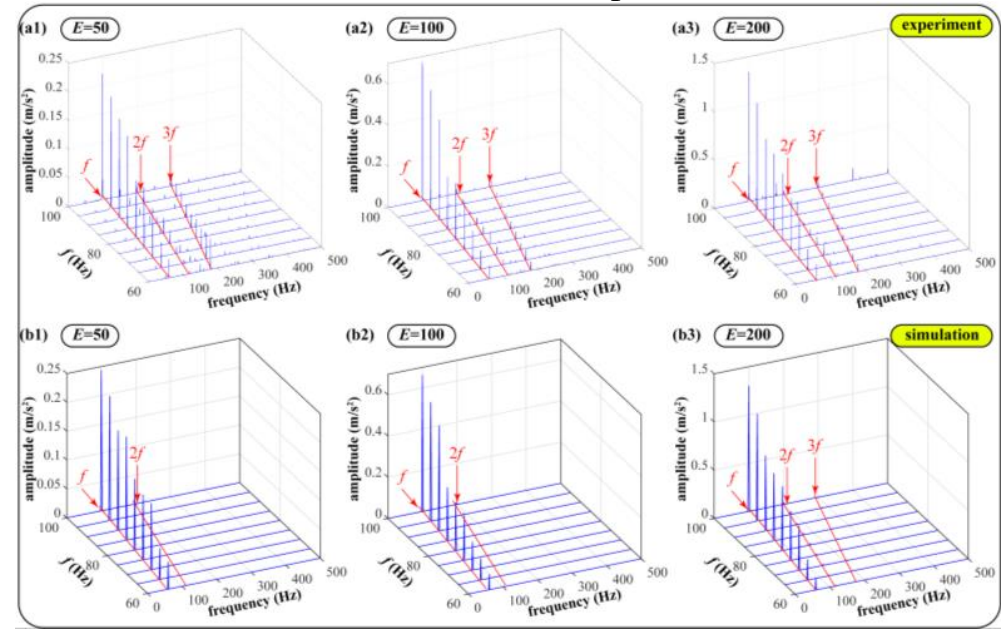

Fig. 9 3-D frequency spectrum comparison between experimental and theoretical results; (a1-a3) experiment when external amplitudes are 50, 100, 200; (b1-b3) simulation when external amplitudes are 50, 100, 200
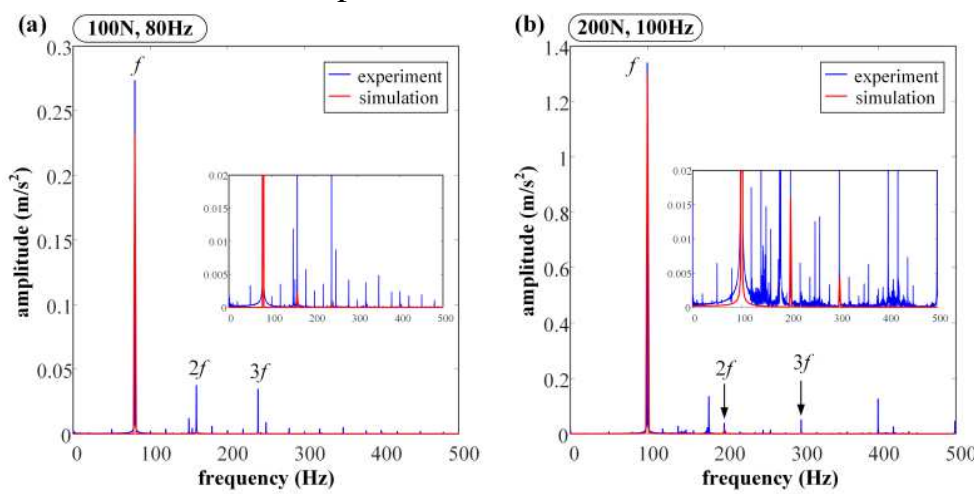

Fig. 10 Detailed spectrum comparison between experimental and theoretical results; (a) excitation with $100 \mathrm{~N}, 80 \mathrm{~Hz}$; (a) excitation with $200 \mathrm{~N}, 100 \mathrm{~Hz}$

With the aid of the impact test arrangement in Fig. 8(b), the FRF (frequency response function) in axial direction of system is extracted, and the results of repeated five times are displayed in Fig. 11(a-b) when paltform is located at $160 \mathrm{~mm}$ and $480 \mathrm{~mm}$, respectively. The detected axial natural frequency varies with the location of screw-nut, but is mostly invariant at 
different times and the same position, as expected. The nine positions in the $0-800 \mathrm{~mm}$ with the interval of $80 \mathrm{~mm}$ are selected to obtain various main peak frequency, and the result is given in Fig. 11(c). It indicates the position-dependent effect of axial natural frequency of system through experiment and proposed model, and the relative errors between measurement and simulation are $0.5 \%, 4.6 \%, 0.7 \%, 3.9 \%, 4.5 \%, 0.8 \%, 7.7 \%, 3.4 \%, 0.1 \%$. The comparison analysis indicates that the proposed model has an ability to predict the dynamic responses of ball screw feed system.
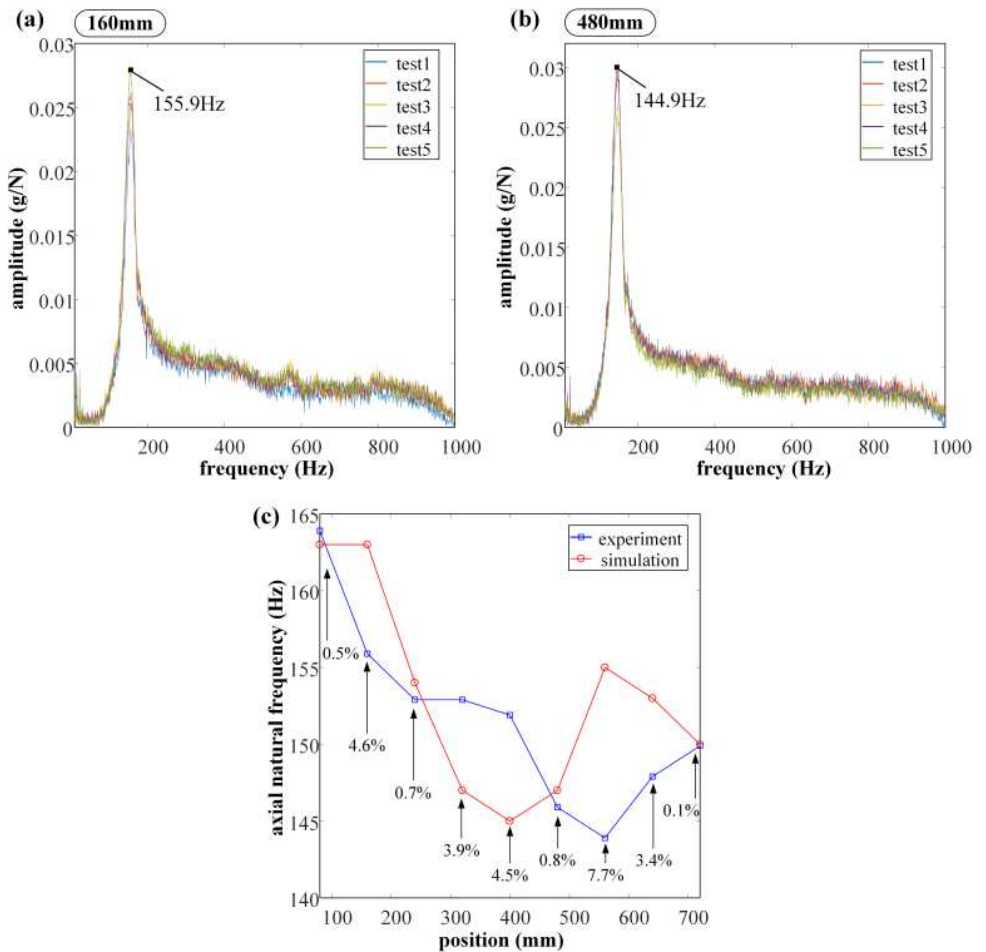

Fig. 11 The results of impact test; (a-b) FRF when platform is loacted at $160 \mathrm{~mm}$ and $480 \mathrm{~mm}$; (c) axial natural frequency comparison between mesured and simulated results

\subsection{Effect of external harmonic excitation on dynamic response}

The response of worktable is output for simulation analysis. Fig. 12 shows the frequency response curves of BSFS in six vibration directions when excitation amplitude $E F_{e 0}=\left[F_{x 0}, F_{y 0}, M_{x 0}\right.$, $\left.M_{y 0}, M_{z 0}\right]$ with unit load $F_{e 0}=[1,1,0.1,0.1,0.1]$ is used and $E=1000,2000,3000$ respectively. The worktable position $L_{p}=400 \mathrm{~mm}$ is fixed. The ordinate value is generated by peak-to-peak value of steady-state solution. The system undergoes resonances in a wide frequency range. As excitation frequency increases, the multiple resonance zones of nonlinear system are excited, and super- and sub-harmonic solutions and jumping phenomenon as the manifestation of nonlinear system occur. The harden effect is illustrated when jumping point bends to the side of increased frequency [43]. Fig. 12(c), (e) and (f) also capture the soften effect when jumping point bends to the side of decreased frequency corresponding to $E=1000$. Taking $E=3000$ for example, there is obvious distinction among the main peaks of frequency sweep plot in six directions. For Fig. 12(a)-(f), the main peak frequencies are located at $1470 \mathrm{~Hz}, 1395 \mathrm{~Hz}, 135 \mathrm{~Hz}, 1320 \mathrm{~Hz}, 2160 \mathrm{~Hz}, 1860 \mathrm{~Hz}$, respectively. The lowest and highest resonance frequencies are in feed and pitching directions. In Fig. 12(c), the peak-to-peak value of main peak in feed direction is higher than vertical and lateral direction due to the fact that axial stiffness of BSFS is the weakest part. Besides, the frequency corresponding to main peak points of six vibration directions can be found in each frequency sweep plot. By the compared results of $E=1000,2000$ and 3000, when excitation amplitude is larger, vibration amplitude increases and jumping point corresponding to higher frequency is obtained. From Fig. 12(c), (e) and (f), the soften effect turns to harden effect at the certain frequency range when larger excitation amplitude is used. 

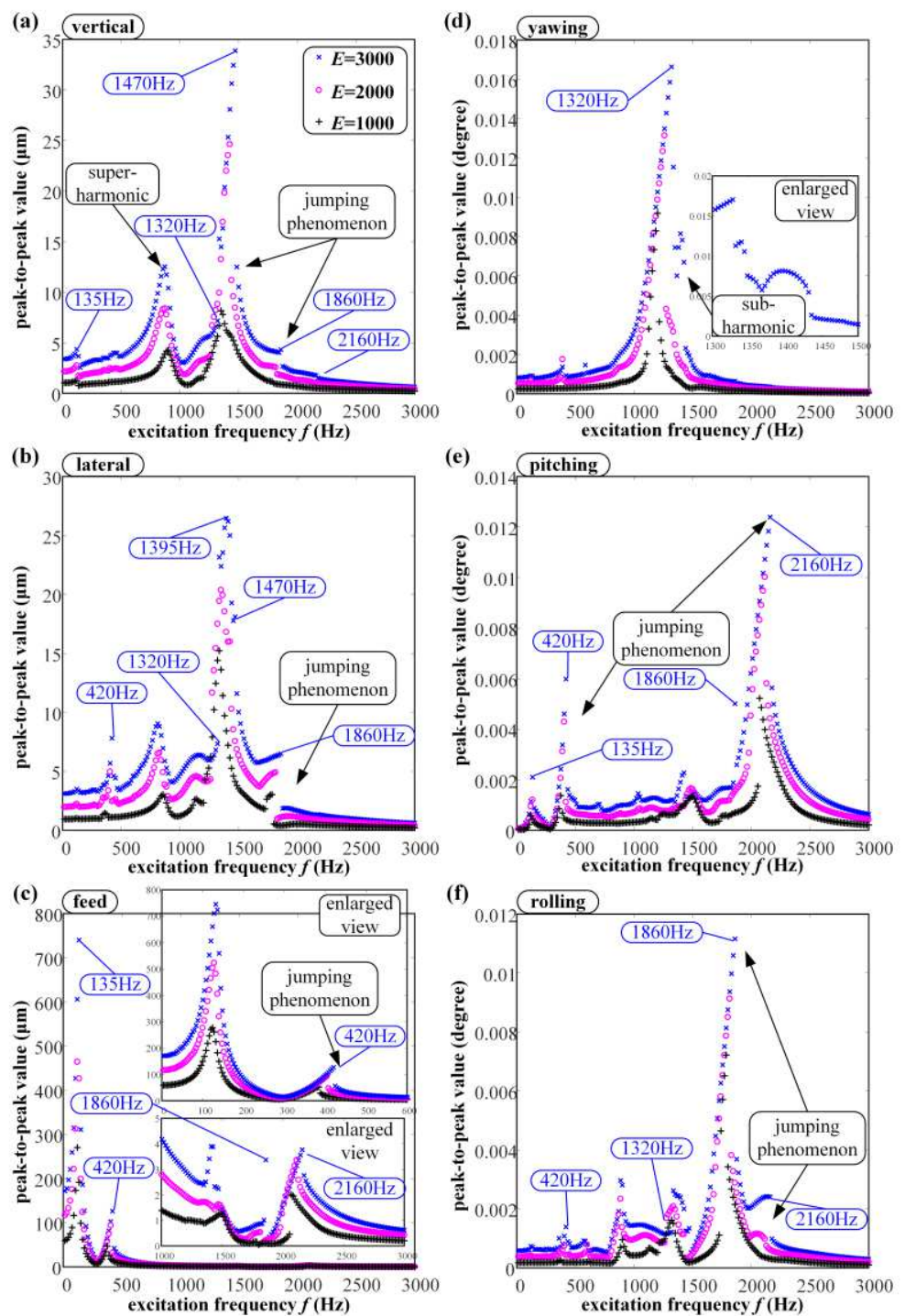

Fig. 12 Frequency sweep plot of six vibration directions under different external load amplitudes

The bifurcation diagram is employed to investigate the stability of BSFS. Although the result of frequency sweep plot exhibits obvious differences in resonance frequency of six vibration directions, bifurcation diagram in Fig. 13 captures the same evolution rule of dynamic behavior in six directions when the control parameter is excitation frequency and $E=3000,5000$ and 10000, respectively. In Fig. 13(c), the main peak frequency in the feed direction is also implied. The jumping discontinuous behavior is displayed, especially for the larger excitation amplitude of $E=10000$. The non-single period motion, such as period- $n$ and quasi-period, indicates that BSFS is in the unstable vibration state. Furthermore, the frequency range corresponding to unstable region expands and moves to the higher frequency when larger $E$ is used. 

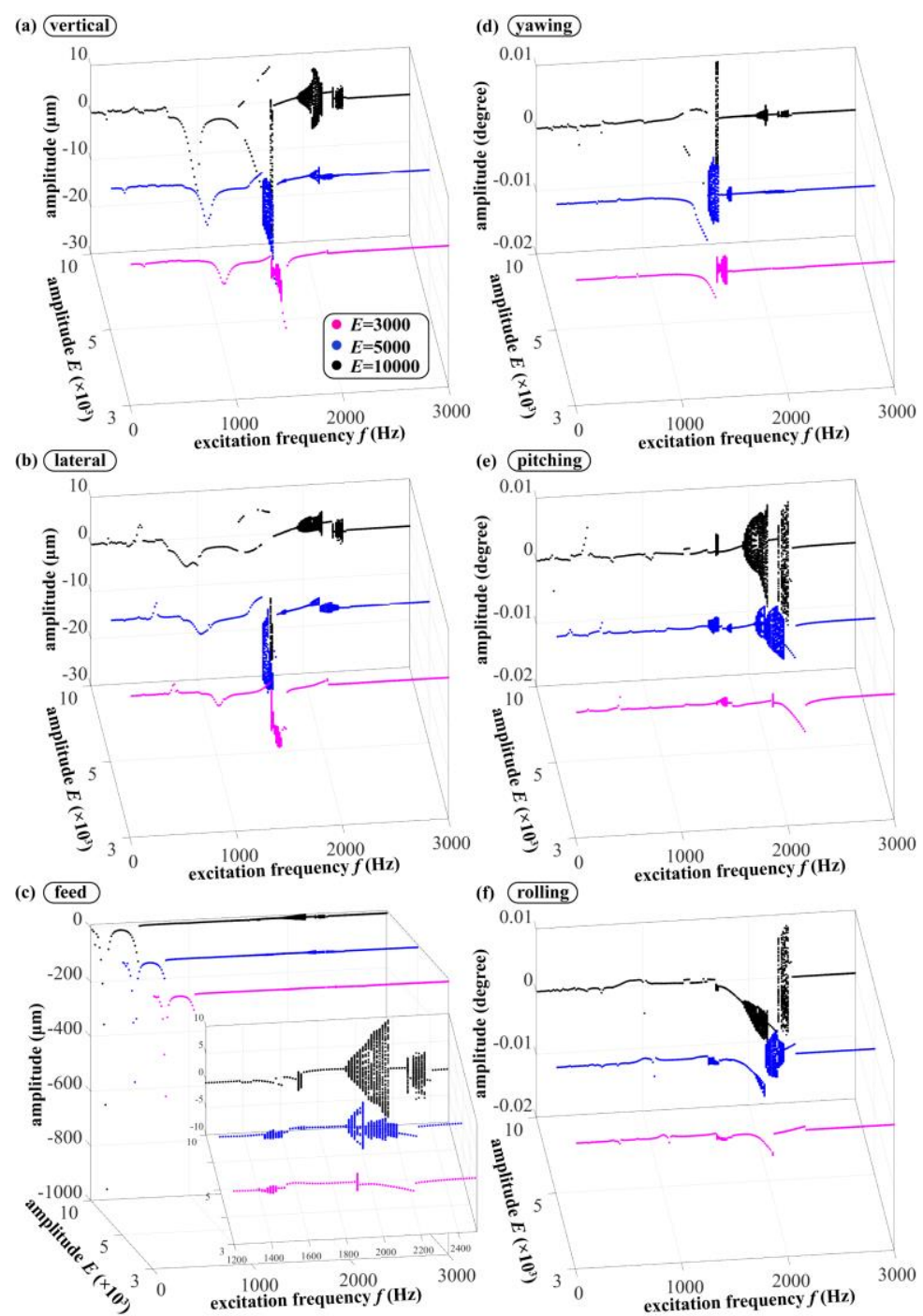

Fig. 13 Bifurcation diagram of six vibration directions with excitation frequency as the control parameter under different excitation amplitude

The response of feed direction is chosen to conduct the next analysis. Fig. 14 shows the bifurcation diagram and corresponding 3-D frequency spectrum with excitation amplitude $E$ as the control parameter. The four typical frequency points $(f=1500 \mathrm{~Hz}, 2000 \mathrm{~Hz}, 2370 \mathrm{~Hz}, 2500 \mathrm{~Hz})$ are selected for exhibition of rich evolution law of dynamic behavior. It can be conlcuded that the nonlinear behavior is sensitive to excitation frequency and amplitude. With the increased excitation amplitude, system tends to the unstable state which behaves as jumping discontinuous, quasi-period and chaos. The specfic motion state is marked in Fig. 14(a1)-(d1). From the result of 3 -D frequency spectrum, the primary frequency $f$ almost dominates the spectrum and its frequency multiplication such as $2 f$ and $3 f$ is also captured. The jumping discontinuous behavior of spectrum is triggered, like non-smooth border of frequency $f$. Besides, the continuous frequency component always appears with quasi-period and chaotic motions. Although the selected wide amplitude scope may be not applied in the engineering, for the purpose of scientific research, the simulation may provide a reference for virtual prototype design and dynamic analysis under extreme condition. 

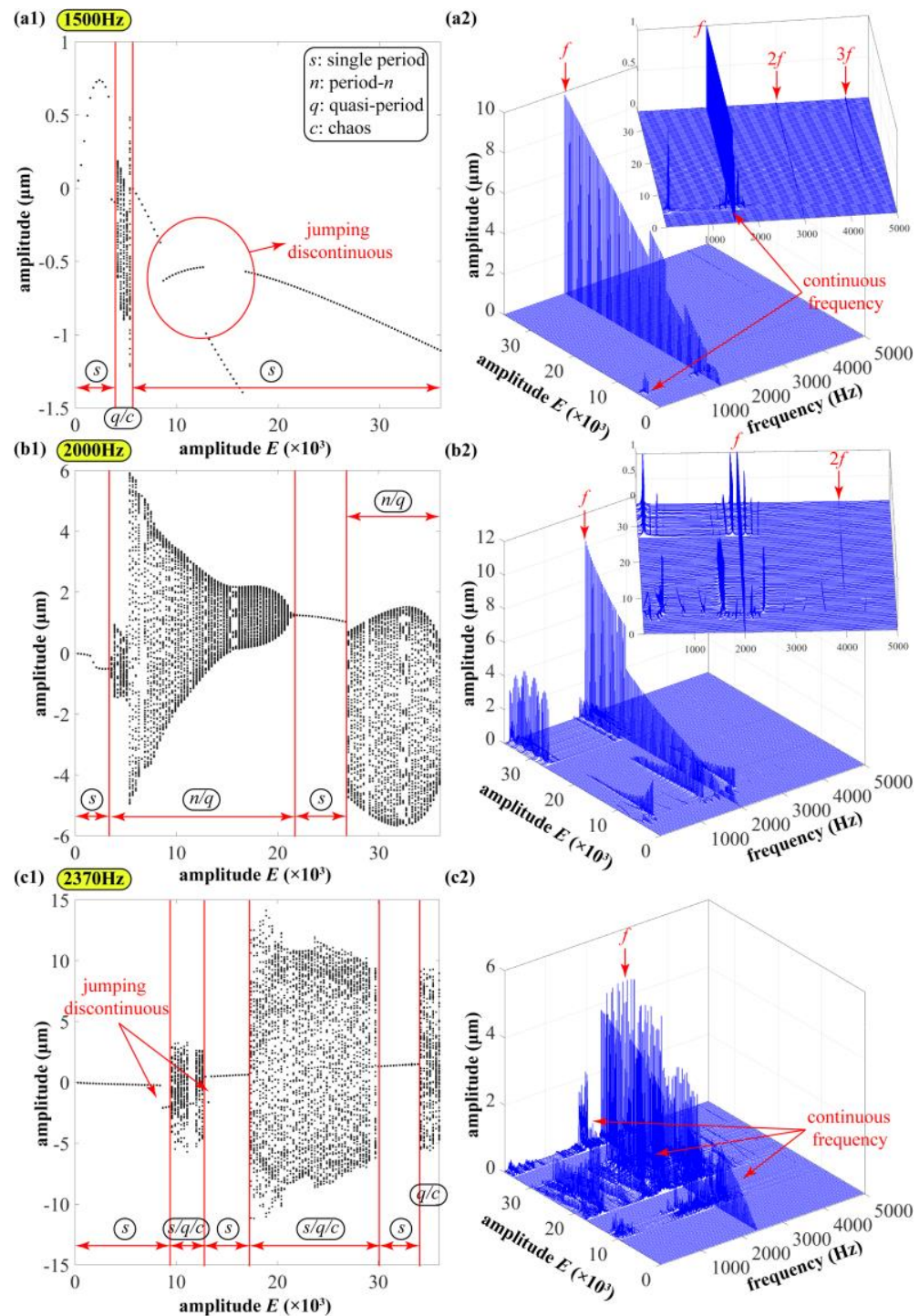

Fig. 14 Bifurcation diagram and 3-D frequency spectrum of feed direction with excitation amplitude as the control parameter under different excitation frequency

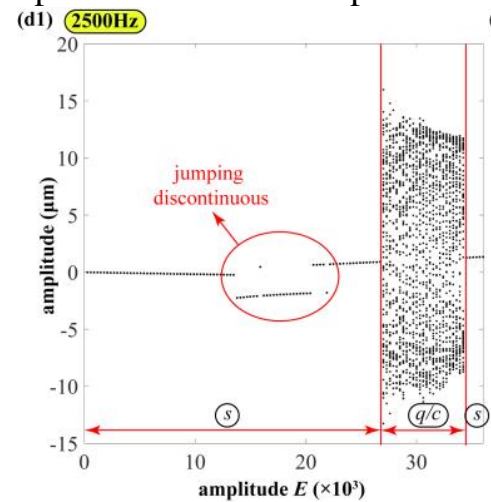

(d2)

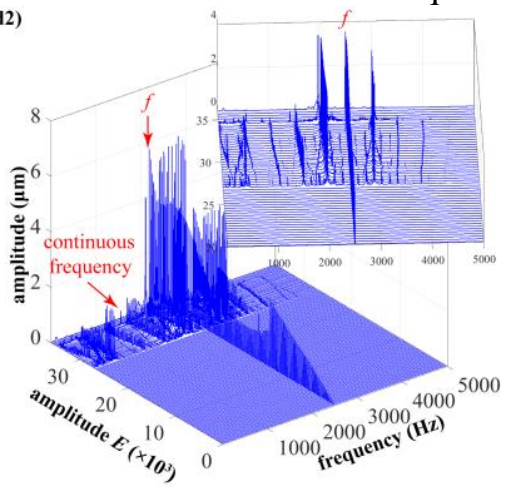

Fig. 14 Continued

Fig. 15 observes eight vibration response windows. At $f=1500 \mathrm{~Hz}$ and $E=5100$, phase trajectories form a ring with a certain width and have differences between adjacent trajectories. There is a closed loop in Poincare map. The frequency domain reveals the non-integer frequency component. It illustrates that system experiences quasi-period motion at present. At $f=1500 \mathrm{~Hz}$ and $E=5400$, Poincaré section shows that chaos is characterized by obvious irregular distribution of Poincaré points and continuous frequency occurs. At $f=2000 \mathrm{~Hz}$ and $E=5400$, quasi-period motion is revealed through a closed loop formed by Poincaré points. In frequency domain, primary frequency $f$ loses its leading position and non-integer frequency $386.7 \mathrm{~Hz}$ and $1613 \mathrm{~Hz}$ become the 
highest peak. At $f=2000 \mathrm{~Hz}$ and $E=6600$, phase trajectories repeat themselves completely every five periods and have no differences between adjacent trajectories. Five isolated points occupy Poincaré section. There are $f / 5,2 f / 5,3 f / 5 \ldots$ in spectrum, and non-integral multiples and continuous frequency disappear. It suggests that 5T-period motion is found. At $f=2370 \mathrm{~Hz}$ and $E=12000$, chaos is captured and its phase trajectories almost fill the entire phase space. Poincaré section is consisted of 3000 irregular distributed Poincaré points. The typical continuous frequency occurs. Further, at $f=2370 \mathrm{~Hz}$ and $E=12600$, chaos evolves into quasi-period motion. The phase trajectories are clearer than the one of chaos. The disordered Poincaré points transit to a closed track. The continuous frequency becomes clearer, but non-integer frequency still exists and leads the spectrum. Similarly, at $f=2500 \mathrm{~Hz}$ and $E=27300,28800$, the same developed phenomena of vibration response is found when chaos evolves into quasi-period motion.

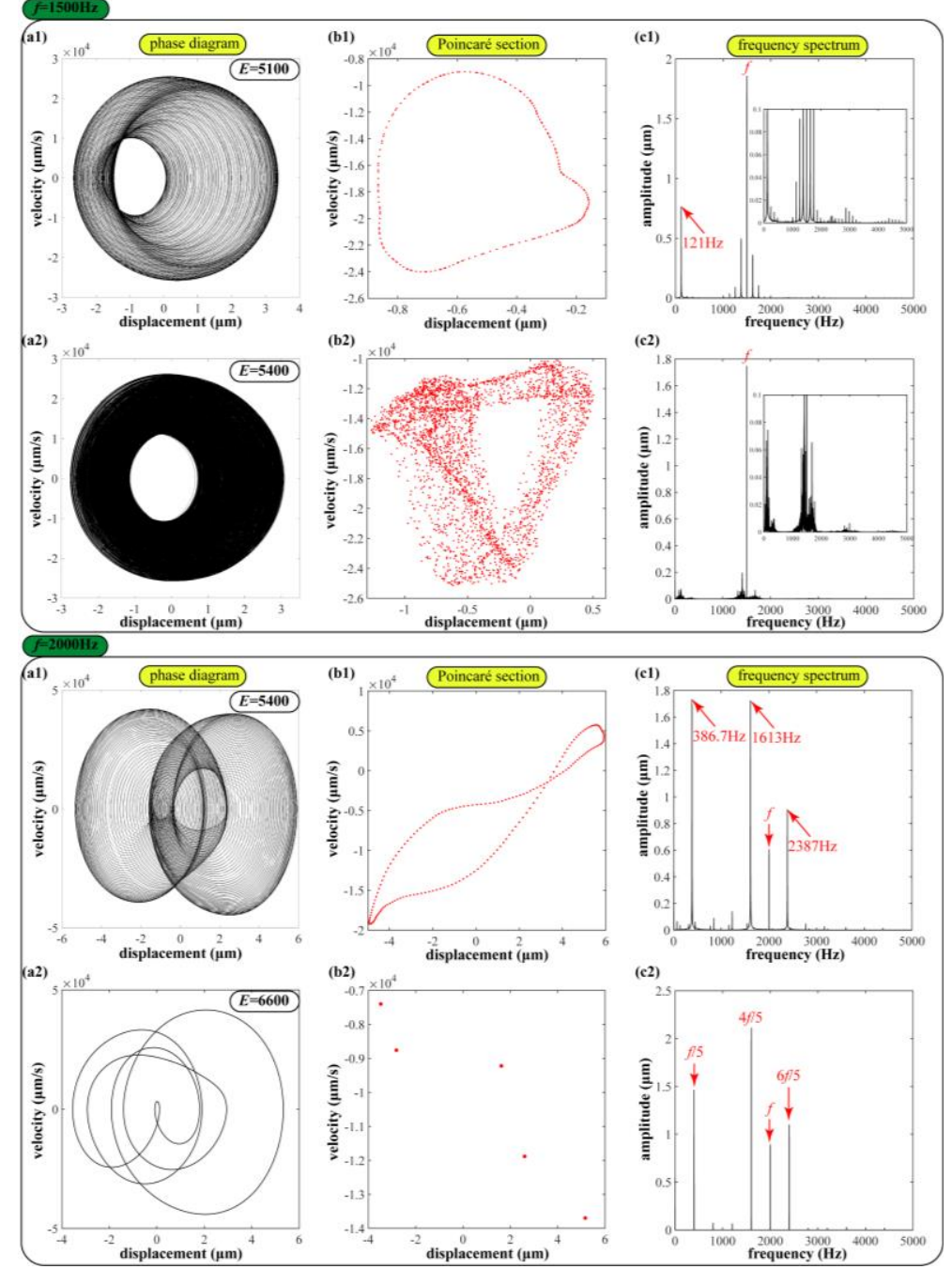

Fig. 15 Vibration response under certain excitation frequency and amplitude 


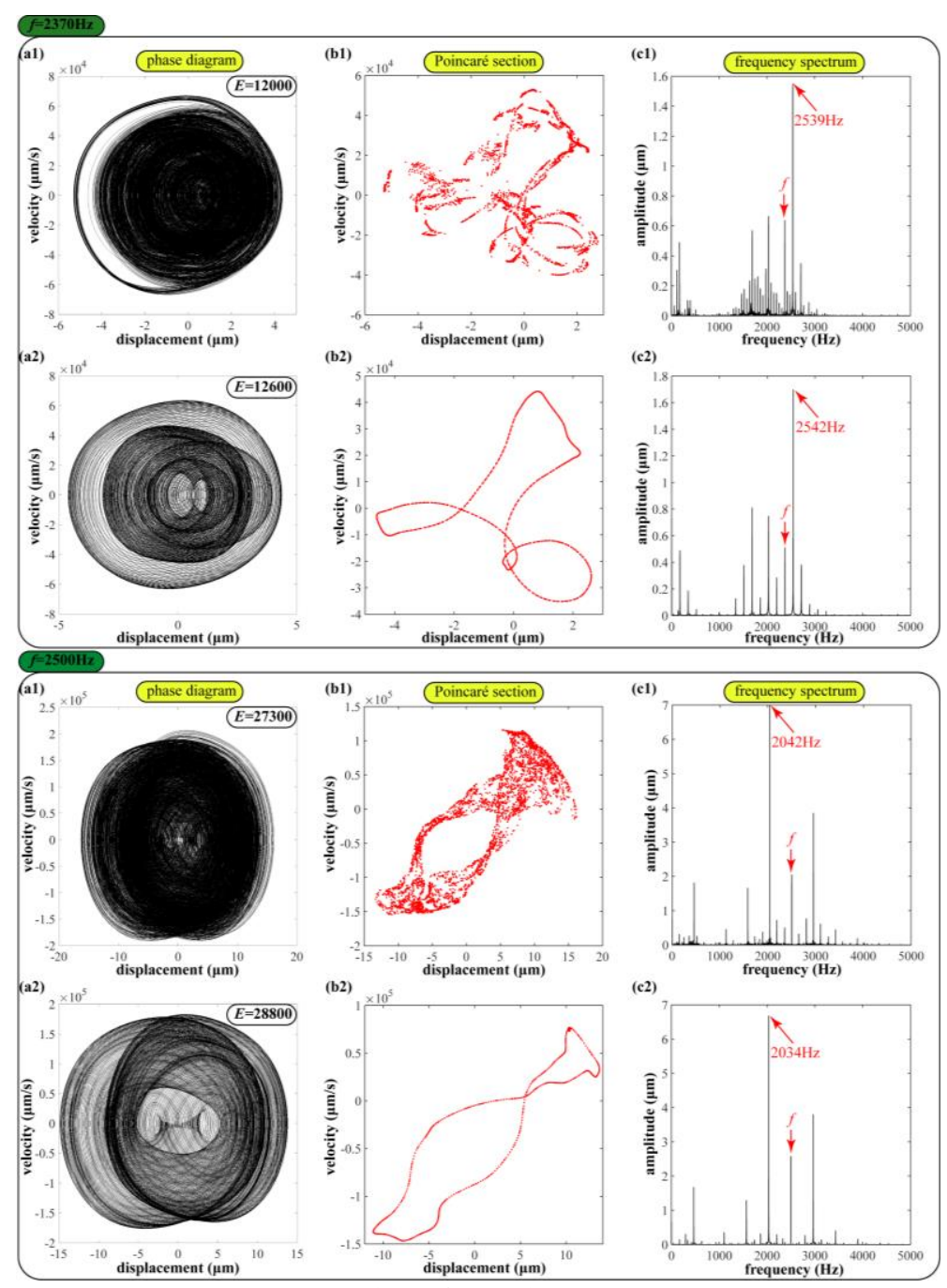

Fig. 15 Continued

\subsection{Effect of worktable position on dynamic response}

In this section, the position-dependent property of BSFS is investigated when excitation amplitude $E=5000$ is fixed and different worktable position $L_{p}$ is used. Fig. 16 shows the bifurcation diagram with excitation frequency as the control parameter when $L_{p}=100$, $200 \ldots .700 \mathrm{~mm}$ and $f \in(0,2200 \mathrm{~Hz})$. In Fig. 16(a1-g1), the main resonance frequency point in the feed direction reveals a significant difference. The lowest frequency is $135 \mathrm{~Hz}$ corresponding to the middle position of screw shaft. As worktable is close to two supported ends, the main resonance frequency increases symmetrically and they are $150 \mathrm{~Hz}$ for 300 and $500 \mathrm{~mm}, 150 \mathrm{~Hz}$ for 200 and $600 \mathrm{~mm}, 165 \mathrm{~Hz}$ for 100 and $700 \mathrm{~mm}$, respectively. The second resonance frequency point is gradually dropping when worktable is moving to radial bearing end from matched bearings. The resonance frequencies are $495 \mathrm{~Hz}, 465 \mathrm{~Hz}, 450 \mathrm{~Hz}, 435 \mathrm{~Hz}, 420 \mathrm{~Hz}, 420 \mathrm{~Hz}, 405 \mathrm{~Hz}$, respectively. When $f \in(1400 \mathrm{~Hz}, 2200 \mathrm{~Hz})$, as shown in Fig. 16(a2-g2), the unstable frequency region is slightly widened as worktable is far from fixed end of matched bearings. It also demonstrates the existence of unstable region when $f \in(0,1400 \mathrm{~Hz})$ and $L_{p}=100$ and $700 \mathrm{~mm}$, as shown in Fig. 16(a1) and (g1). The detailed development process of nonlinear behavior is displayed in Fig. 17. Only single-period motion is captured when $f \in(0,1400 \mathrm{~Hz})$ and $L_{p}=200,300 \ldots 600 \mathrm{~mm}$. 

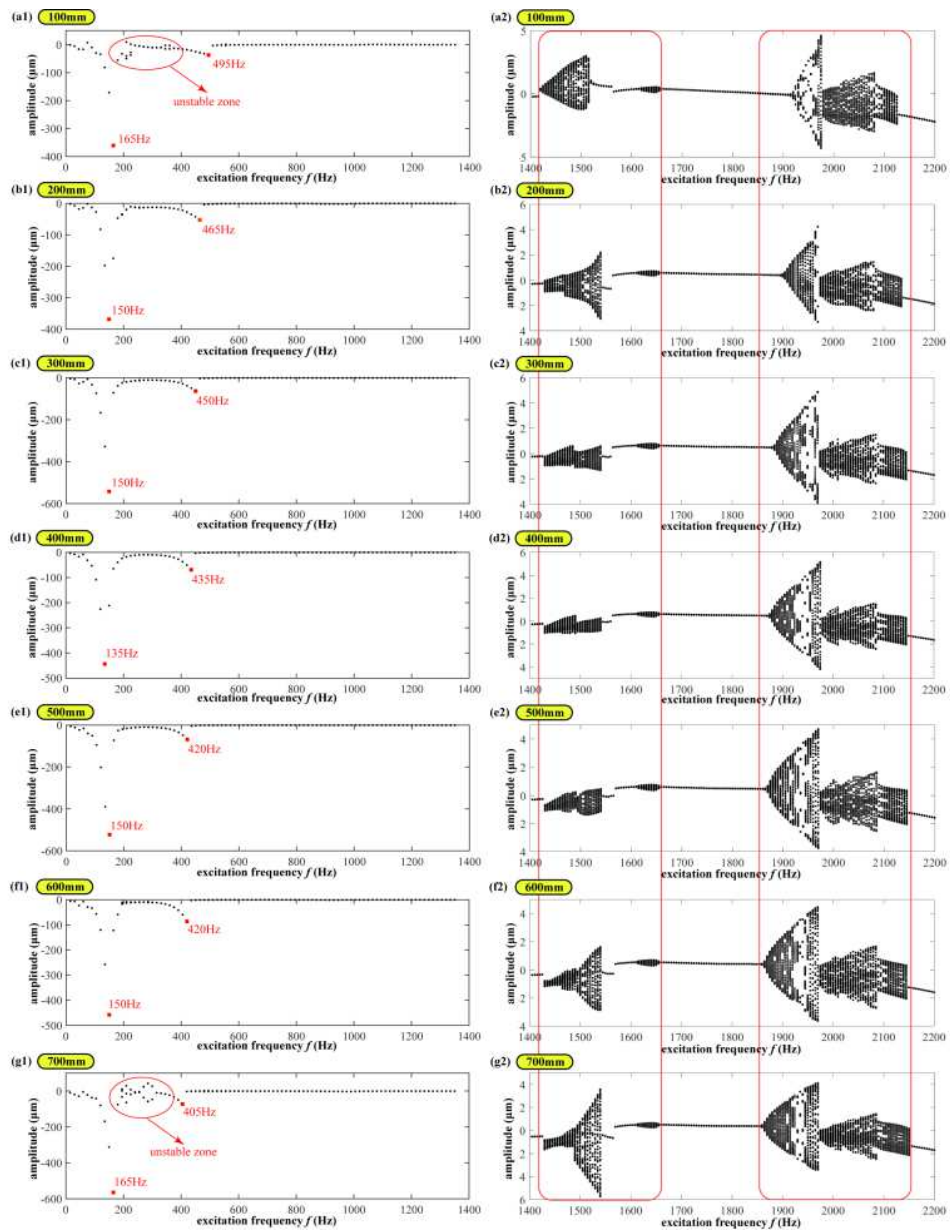

Fig. 16 Bifurcation diagram with excitation frequency as the control parameter under different worktable position when excitation amplitude $E=5000$
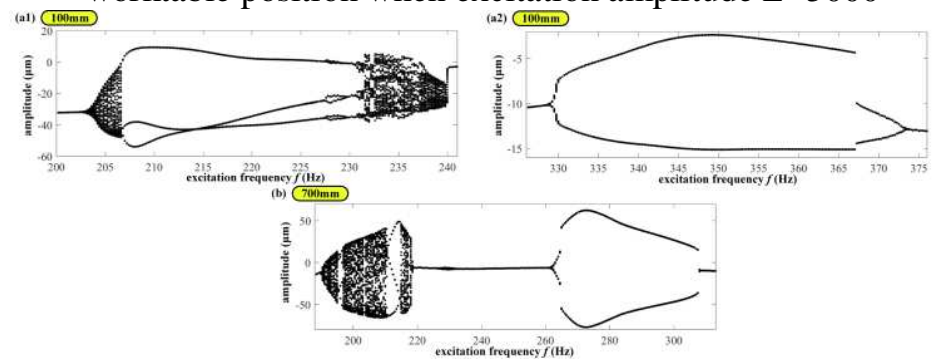

Fig. 17 Partial bifurcation diagram with excitation frequency as the control parameter at $L_{p}=100$ and $700 \mathrm{~mm}$ when $E=5000$

From Fig. 17(a1), the six detailed responses are selected to observe the evolution of orbit and Poincare section with the increased excitation frequency, and the result is depicted in Fig. 18. It is a complete process for a stable period-3 transiting into chaotic motion. The distinct process is formed through period- 3 of $f=224 \mathrm{~Hz}$, period- 6 of $f=228 \mathrm{~Hz}$, period- 12 of $f=231 \mathrm{~Hz}$, quasi-period of $f=231.4 \mathrm{~Hz}$, chaotic motions of $f=231.8$ and $232 \mathrm{~Hz}$. It is a kind of common evolution path that changes a stable periodic motion into chaos. Chaos happened in BSFS means that system works in a state of unpredictability and non-repeatability. For the actual mechanical equipments, chaos is partly detrimental to the working performance and causes noises. According to the bifurcation diagram, the frequency range that probably causes the chaotic motion should be avoided in order to ensure the stability and reliability of system [44]. 


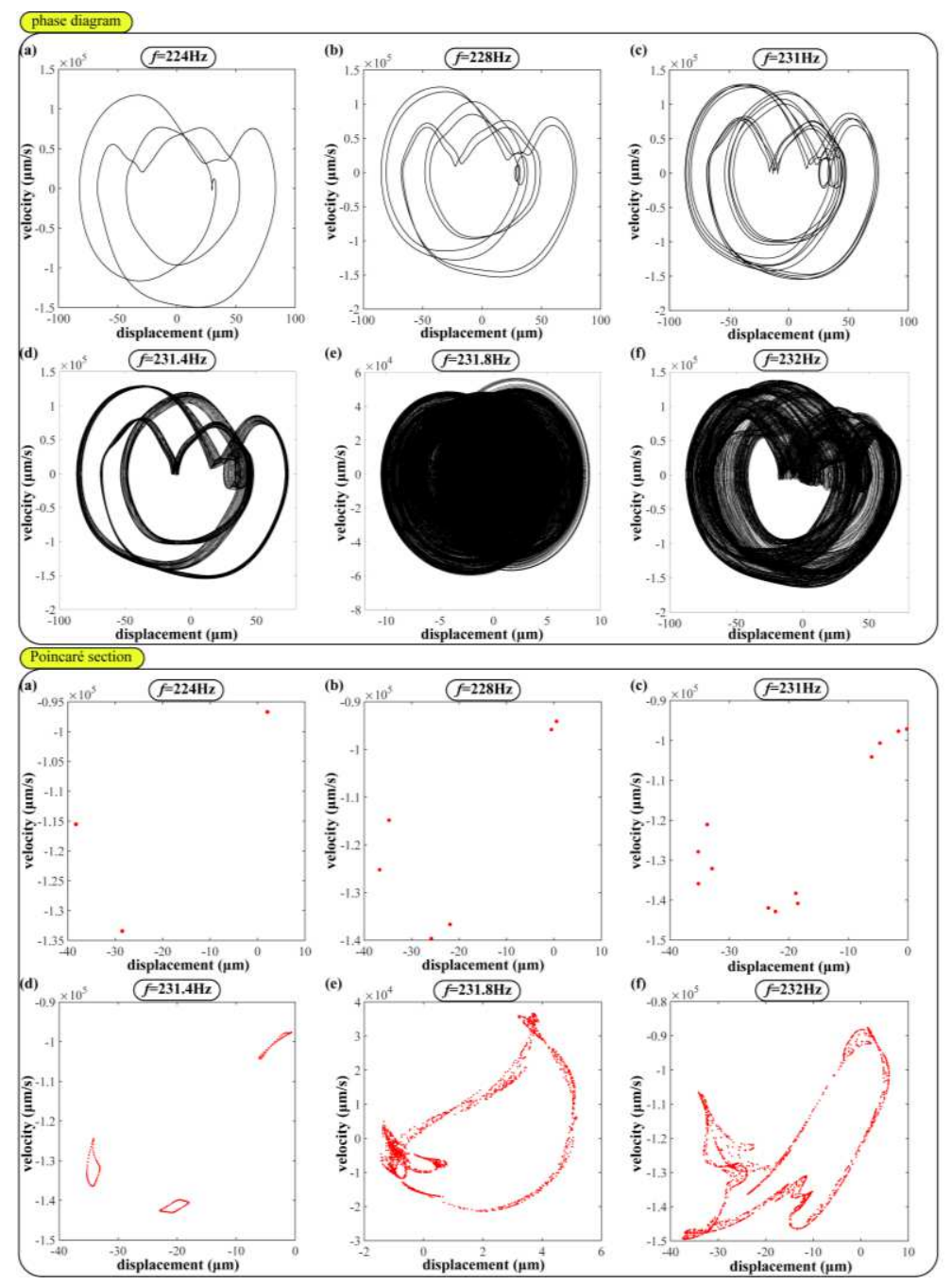

Fig. 18 The evolution of orbit and Poincaré section under different excitation frequency when $L_{p}=100 \mathrm{~mm}$ and $E=5000$

When platform position $L_{p}$ is chosen as the control parameter and harmonic excitation is $f=206 \mathrm{~Hz}$ and $E=5000$, bifurcation and 3-D spectrum are displayed in Fig. 19. It can be concluded that BSFS tends to lose the stability and period- $n$ and quasi-period motions appear when platform is located at the both end of screw shaft. At $L_{p} \in(200 \mathrm{~mm}, 600 \mathrm{~mm})$, Fig. 19(a) indicates that BSFS goes through single-period motion and only frequency $f$ and its integer multiples occur in spectrum of Fig. 19(b). Additionally, period- $n$ motion is always accompanied by $f / n$ frequency components, such as period- 2 motion and $f / 2$, period-3 motion and $f / 3,2 f / 3$, as shown in Fig. 19(b) and 19(a1-a2). 


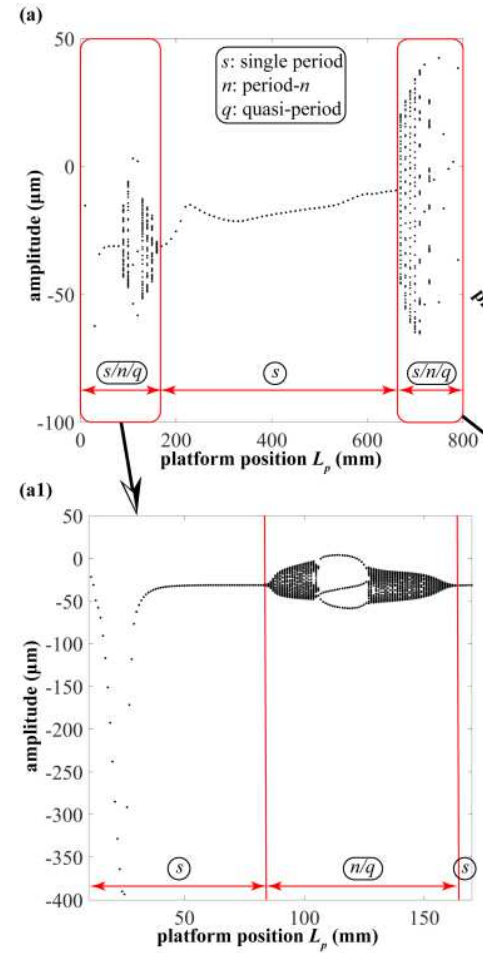

(b)

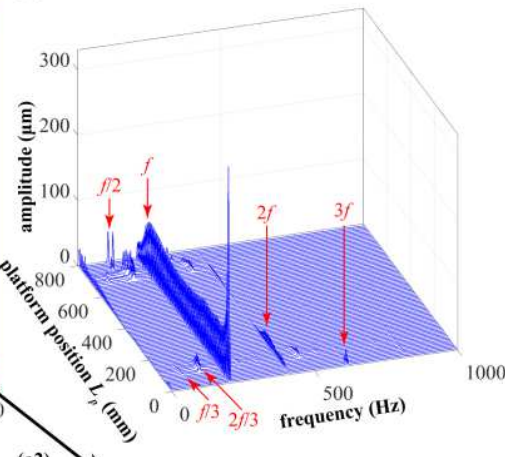

(a2)

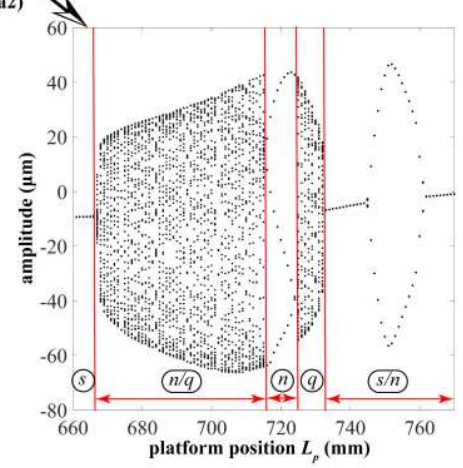

Fig. 19 Bifurcation diagram and 3-D frequency spectrum with worktable position as the control parameter when excitation $f=206 \mathrm{~Hz}$ and $E=5000$

\subsection{Effect of screw shaft length on dynamic response}

The screw length $L \in(0.5 \mathrm{~m}, 1.5 \mathrm{~m})$ is chosen as the control parameter, and various external excitation and fixed platform position of $L_{p}=L / 2$ are employed. In Fig. 20(a1-a3), when higher excitation amplitude $E$ is used, the screw length region corresponding to unstable motion is enlarged, and chaotic motion is even captured. From the bifurcation diagrams of Fig. 20(b1), (a1), (b2) and (b3), the jumping discontinuous, quasi-period, chaos and single-period are found when $f$ is $1500 \mathrm{~Hz}, 2000 \mathrm{~Hz}, 2370 \mathrm{~Hz}$ and $2500 \mathrm{~Hz}$ respectively. In the corresponding 3-D frequency spectrum, fundamental frequency $f$ dominates the frequency domain, and diverse frequencies are captured which demonstrate the rich nonlinear behavior with the variation of screw length. The parameter range of continuous frequency is consistent with chaotic motion area. The screw length has a substantial impact on the nonlinear behavior of BSFS. However, the regular evolution law is hardly generalized with the variation of screw length since dynamic characteristic is sensitive to external excitation. The four detailed responses from Fig. 20(a3) and (b2) are selected for the observation of quasi-period and chaotic motions, and the result is depicted in Fig. 21. 

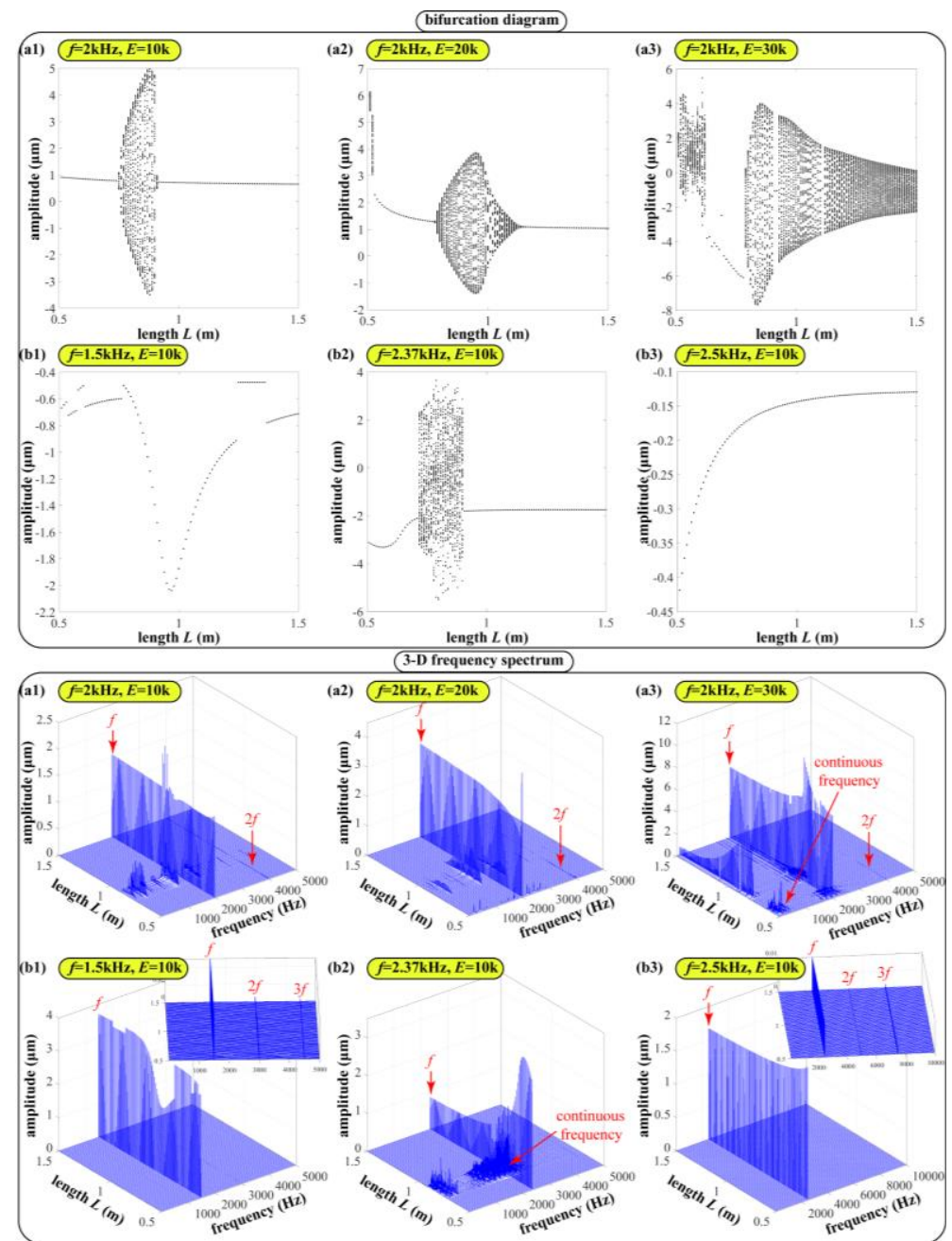

Fig. 20 Bifurcation diagram and 3-D frequency spectrum with screw shaft length as the control parameter under different external excitation 

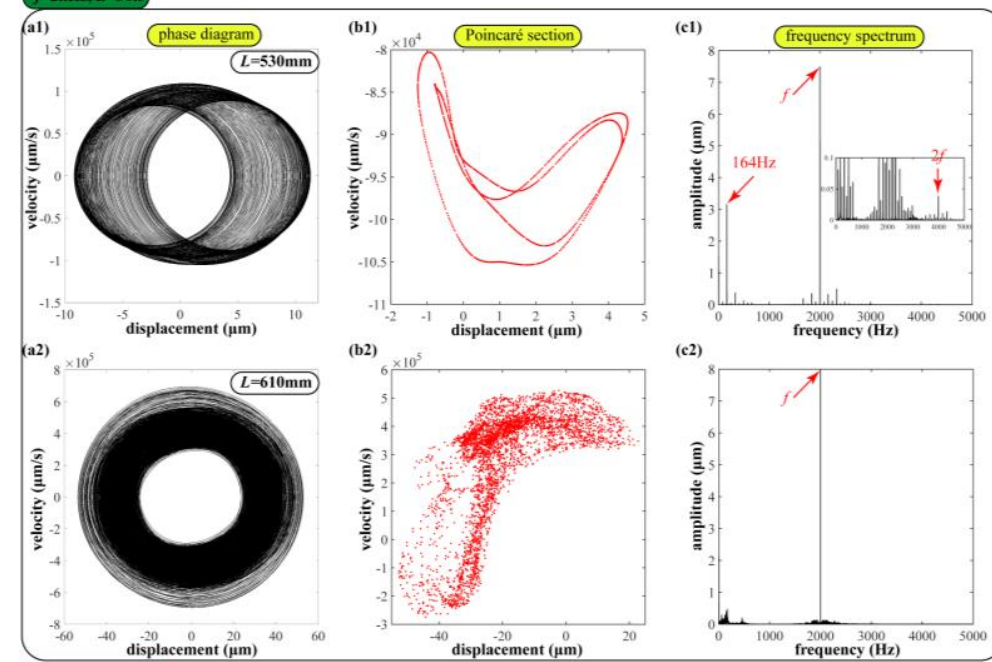

(c2)
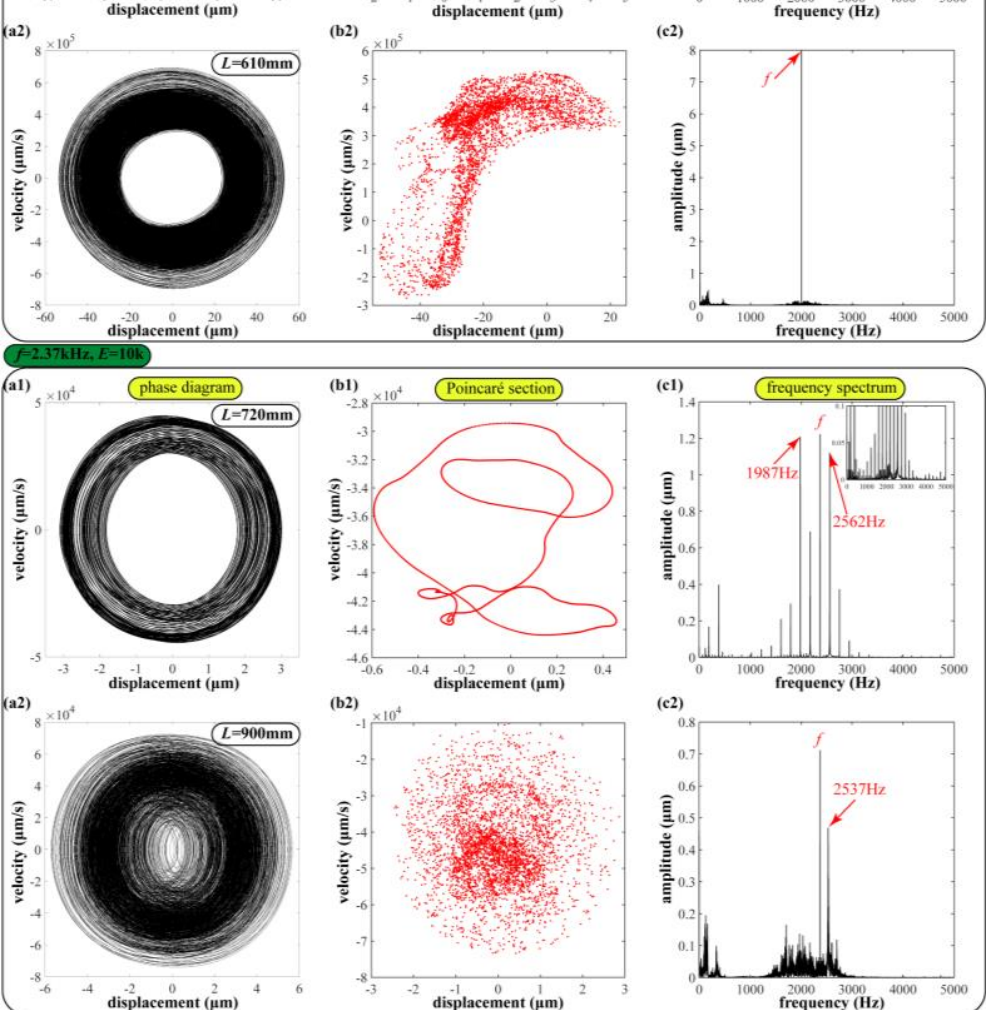

Fig. 21 Vibration response under certain excitation and screw shaft length

\subsection{Effect of preload on dynamic response}

\subsubsection{The influence of carriage preload}

Platform supported by four carriages bears the most of vertical and lateral loads and torque. The carriage preload is the main factor that affects the stiffness and dynamic characteristics of BSFS. Fig. 22 exhibits the bifurcation diagram and 3-D spectrum when carriage preload is chosen as the control parameter, $f=2000 \mathrm{~Hz}, L=800 \mathrm{~mm}, L_{p}=L / 2$ and $E=10 \mathrm{k}, 20 \mathrm{k}, 30 \mathrm{k}$, respectively. For Fig. 22(a1-a2), system develops into the stable single-period from quasi-period when larger carriage preload is employed. The frequency component except $f$ and its multiples gradually disappears with the increased carriage preload, and basic frequency $f$ leads the frequency domain. For Fig. 22(b1-b2) and (c1-c2), when larger amplitude $E$ is employed, the unstable region is expanded and jumping discontinuous is observed from bifurcation diagram and frequency component $f$. The conclusion is similar to that in the above section. Besides, in the spectrums of Fig. 22(b2-c2), the highest frequency is still basic frequency $f$. 


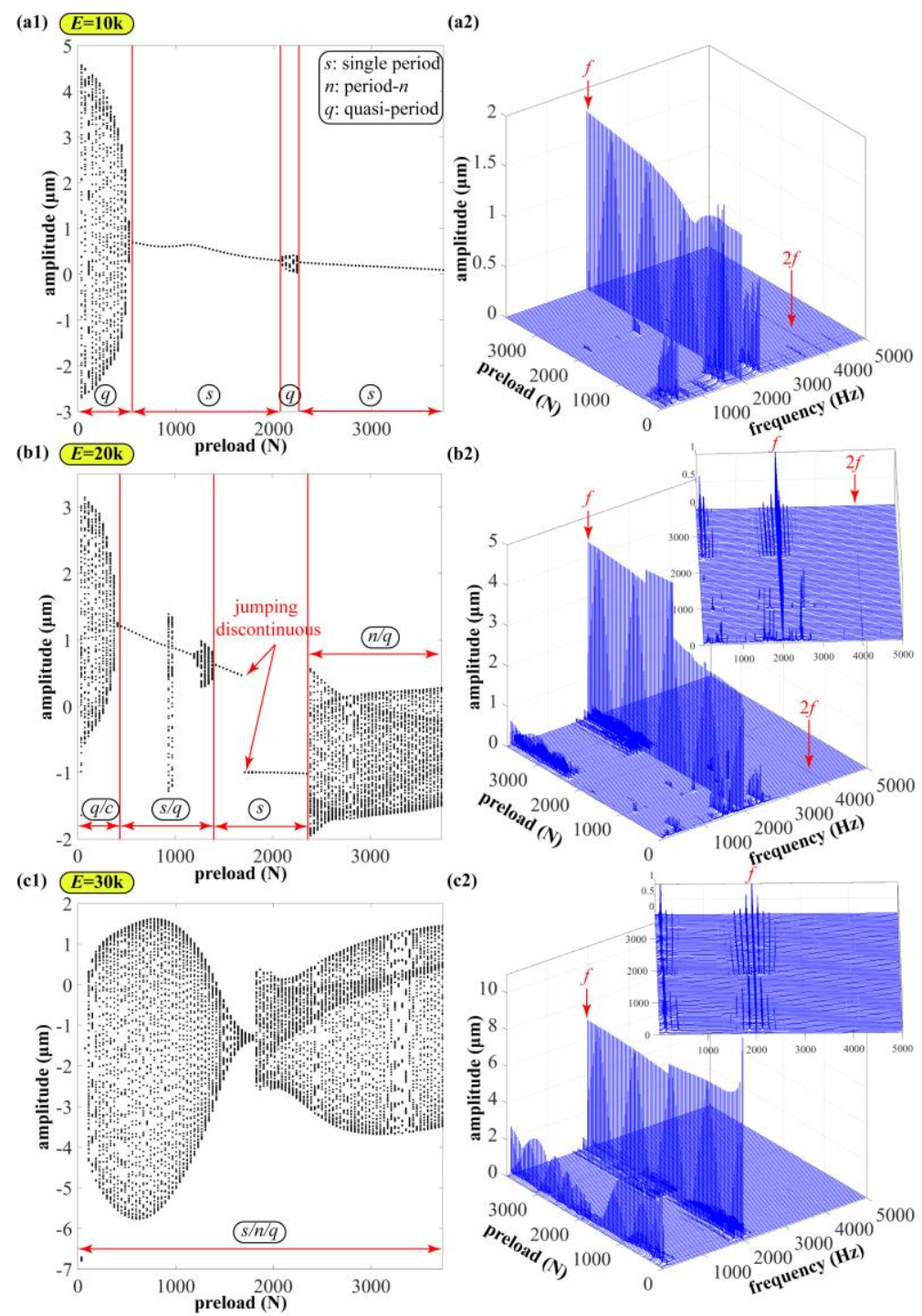

Fig. 22 Bifurcation diagram and 3-D frequency spectrum with carriage preload as the control parameter under different excitation amplitide when $f=2 \mathrm{kHz}$

\subsubsection{The influence of nut preload}

The nut preload is taken as the control parameter and other condition is the same as section 3.5.1. When $E=10 \mathrm{k}$ in Fig. 23(a1-a2), system evolves from single-period through quasi-period to single-period motion. In spectrum, jumping fails to be captured and non-integer multiple frequency occurs. When $E=20 \mathrm{k}$ in Fig. 23(b1-b2), system develops into single-period from quasi-period. At preload $\in(0,2016 \mathrm{~N})$, several single-period and period- $n$ motion windows are observed. When $E=30 \mathrm{k}$ in Fig. 23(c1-c2), as preload increases, system transforms to stable single-period from period- $n$ and quasi-period motions. Besides, larger excitation amplitude triggers the jumping phenomenon in the bifurcation diagram and spectrum. The characteristic of period-31 motion is illustrated in Fig. 24 when preload is $1050 \mathrm{~N}$ and $E=30 \mathrm{k}$. The phase trajectories repeat themselves completely every 31 periods. Poincaré section is formed by 31 discrete points. The shape is similar to quasi-period motion, but a closed curve loop fails to appear. There are $2 f / 31,29 f / 31 \ldots$ in the frequency domain, and non-integral multiples and continuous frequency disappear. 

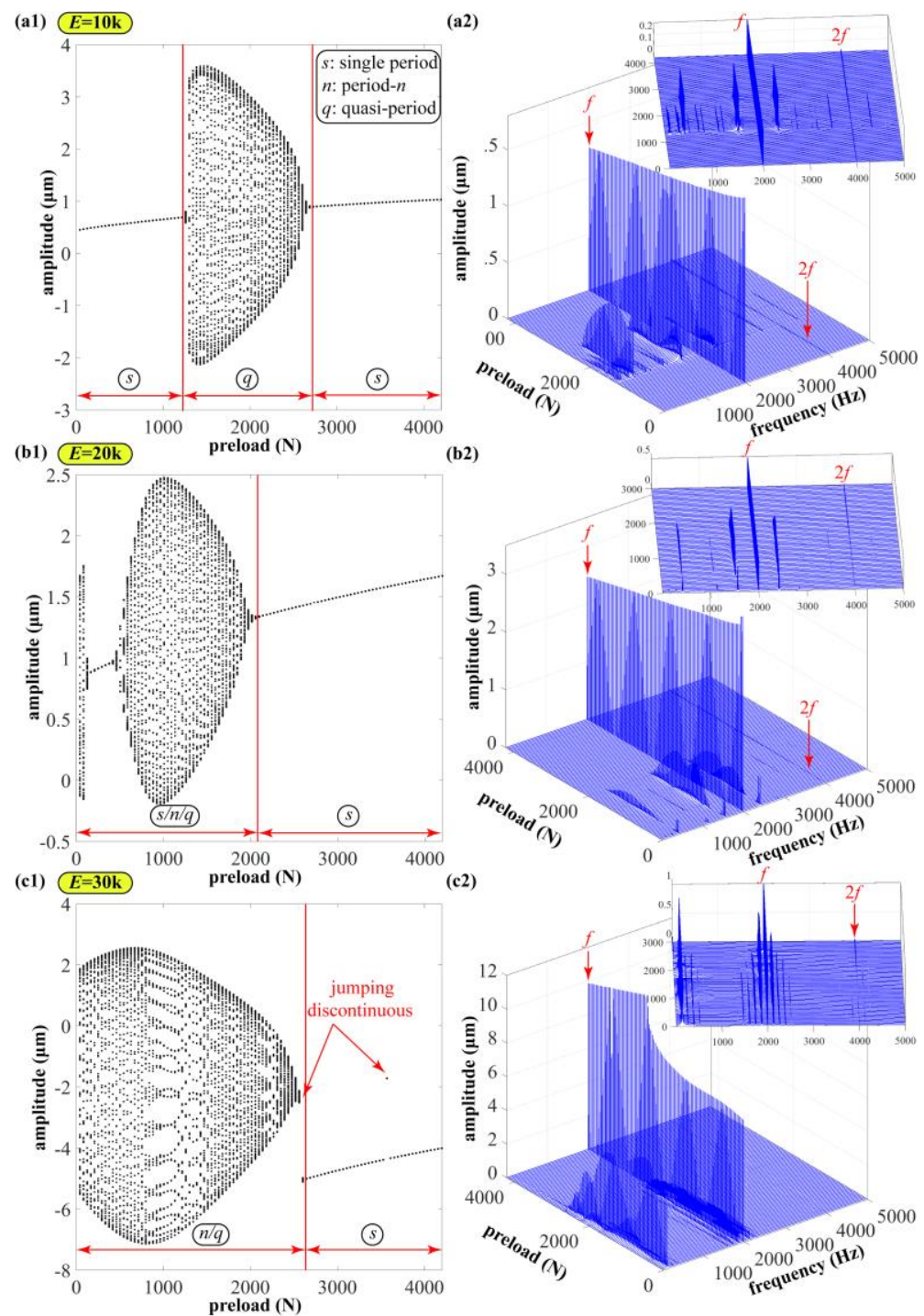

Fig. 23 Bifurcation diagram and 3-D frequency spectrum with nut preload as the control parameter under different excitation amplitide when $f=2 \mathrm{kHz}$
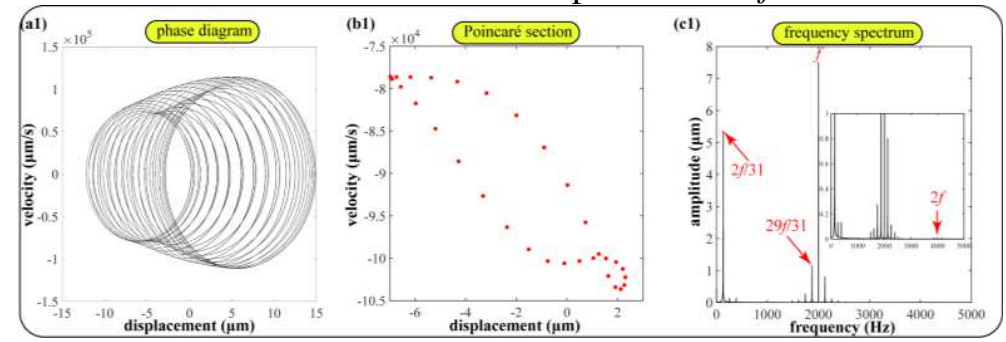

Fig. 24 Vibration response of 31T-period motion when excitation $f=2 \mathrm{kHz}, E=30 \mathrm{k}$ and nut preload

\subsubsection{The influence of matched bearing preload}

\section{is $1050 \mathrm{~N}$}

The effect of matched bearing preload on stability of BSFS is investigated through bifurcation diagram and 3-D frequency spectrum and other conditions are consistent with preceding section. From the result of Fig. 25, the system shows unstable period- $n$ and quasi-period responses at low values of preload. As preload increases, system transforms to stable single-period motion. Furthermore, at $E=30 \mathrm{k}$, the discontinuous behavior of bifurcation is captured. The results demonstrate the effectiveness of larger preload in suppressing non-period motions of BSFS, thereby improving its dynamic response. 


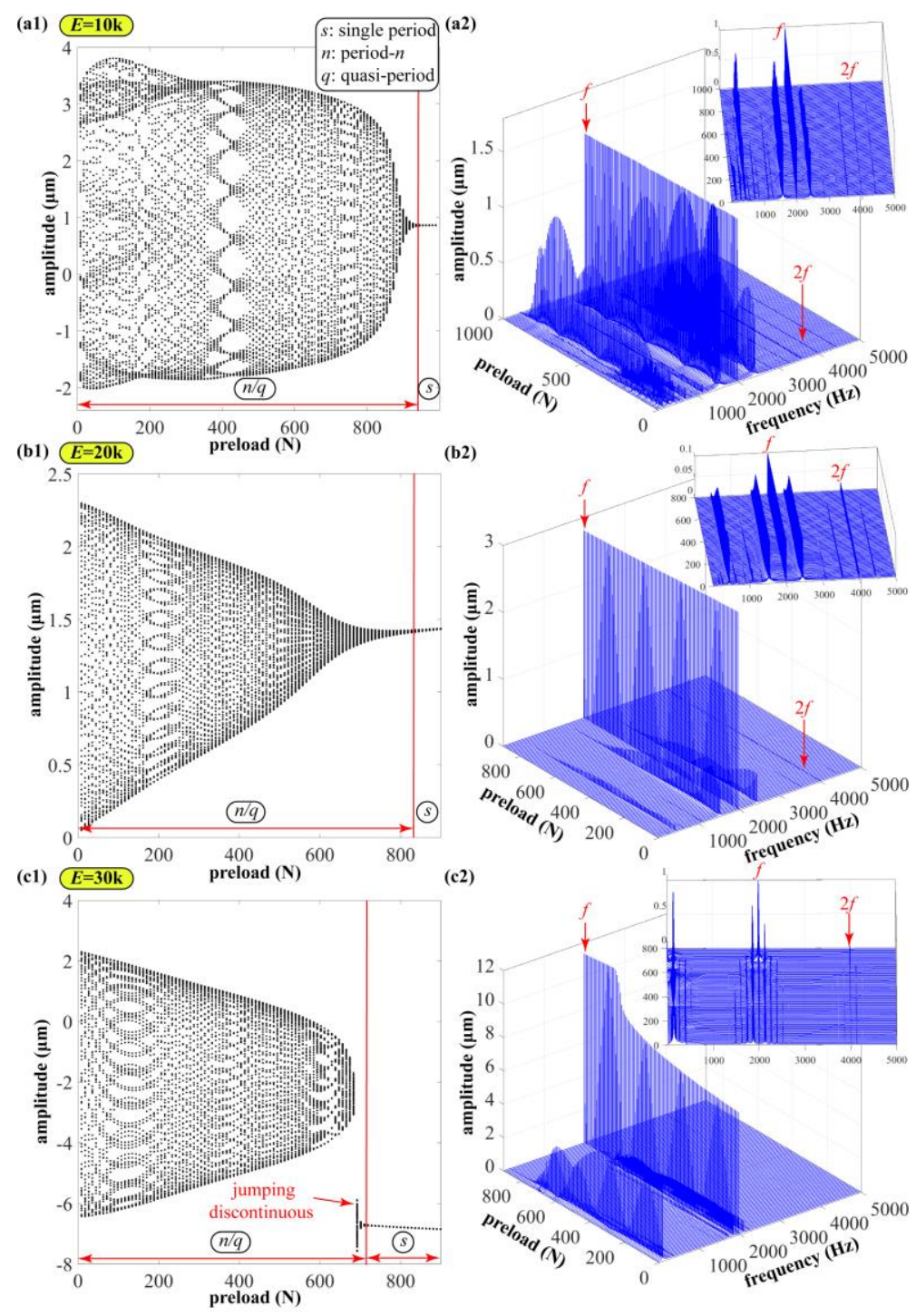

Fig. 25 Bifurcation diagram and 3-D frequency spectrum with matched bearings preload as the control parameter under different excitation amplitide when $f=2 \mathrm{kHz}$

\subsection{Effect of damping on dynamic response}

In this section, damping coefficient between rolling interfaces is used as the control parameter when $f=2 \mathrm{kHz}, E=5000, L=800 \mathrm{~mm}, L_{p}=400 \mathrm{~mm}$, preloads of carriage, nut and bearing are $623 \mathrm{~N}, 1690 \mathrm{~N}$ and $290 \mathrm{~N}$. The system reveals the stable single-period motion at the large value of damping. At the low range of damping coefficient, for carriage and nut, the period- $n$ and quasi-period motions are found by Fig. 26(a1-b1). The spectrum shows a rich component including non-integer and integer multiples, and the amplitude of primary frequency $f$ changes nonlinearly. For matched bearings of Fig. 26(c1-c2), at damping $\in(0,320)$, a non-periodic chaotic motion is captured and continuous frequency is found in spectrum. With the increased damping, period- $n$ and quasi-period motions appear alternately at $(320,8240)$. Then, system enters single-period motion and non-integer multiples disappear in spectrum. The corresponding detailed response windows are exhibited by Fig. 27. The common evolution path from chaos through quasi-period to period-19 motion is displayed. By on-purpose designing, the proper damping coefficient could be acquired through adjusting the related structure and the physical parameters of BSFS. As a result of that, the system is expected to have narrow interval of chaotic motion, extended life time, enhanced reliability, and lower noise. 

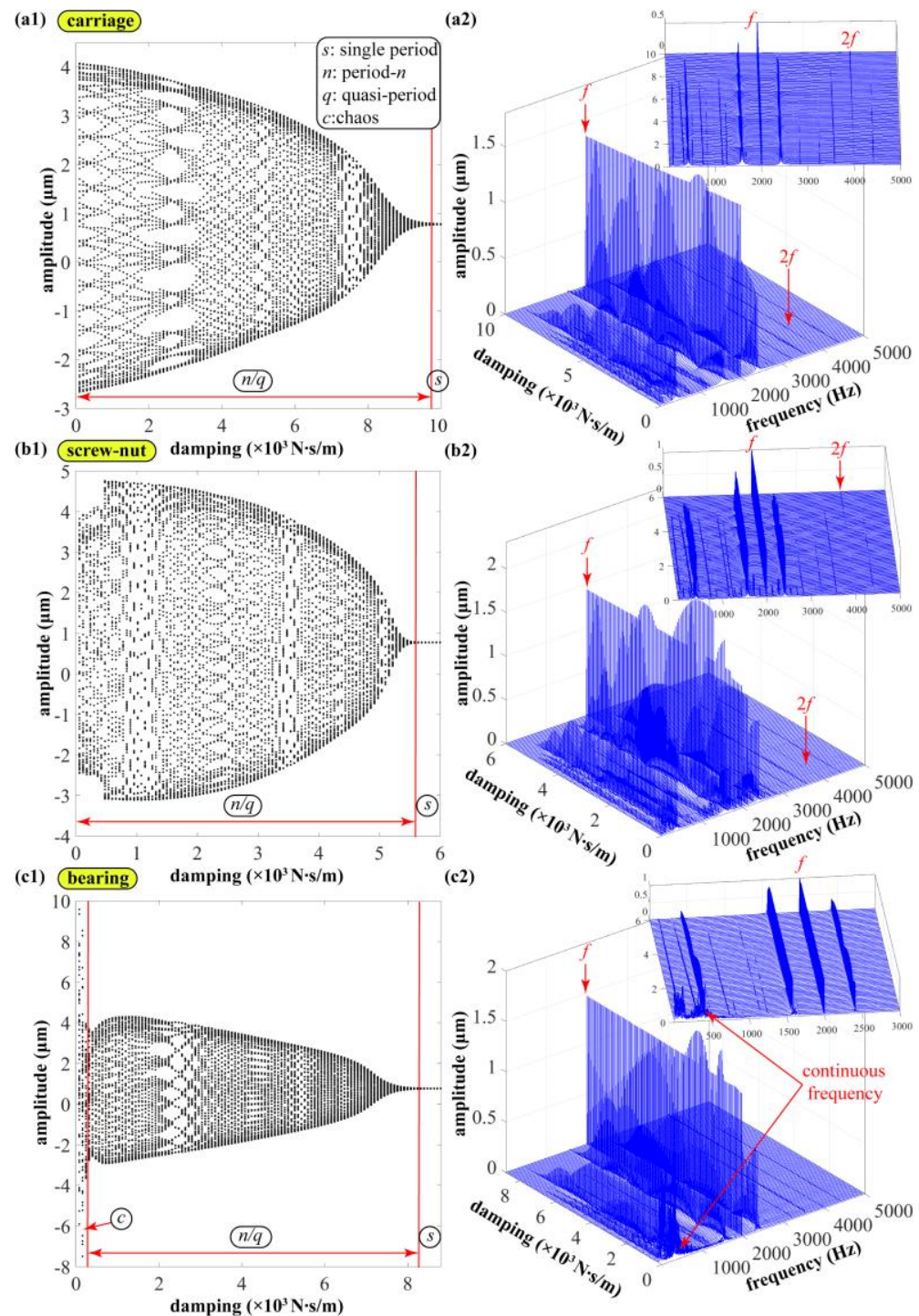

(c2)
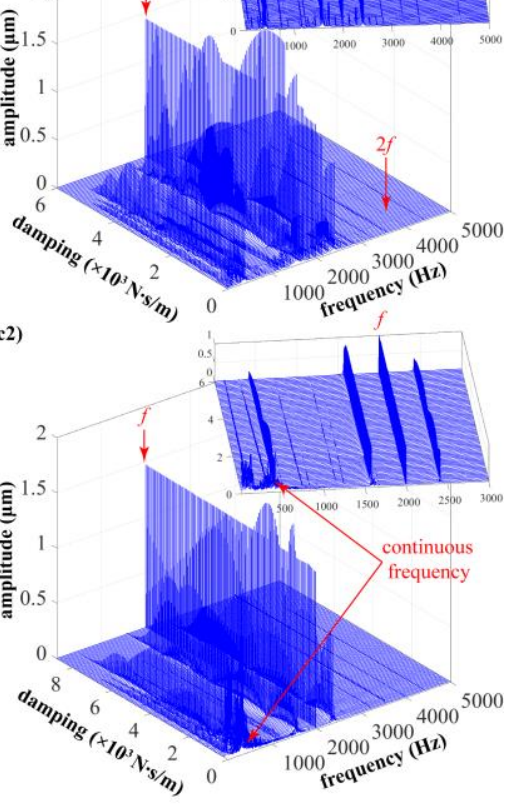

Fig. 26 Bifurcation diagram and 3-D frequency spectrum with damping coefficient of rolling joints as the control parameter 


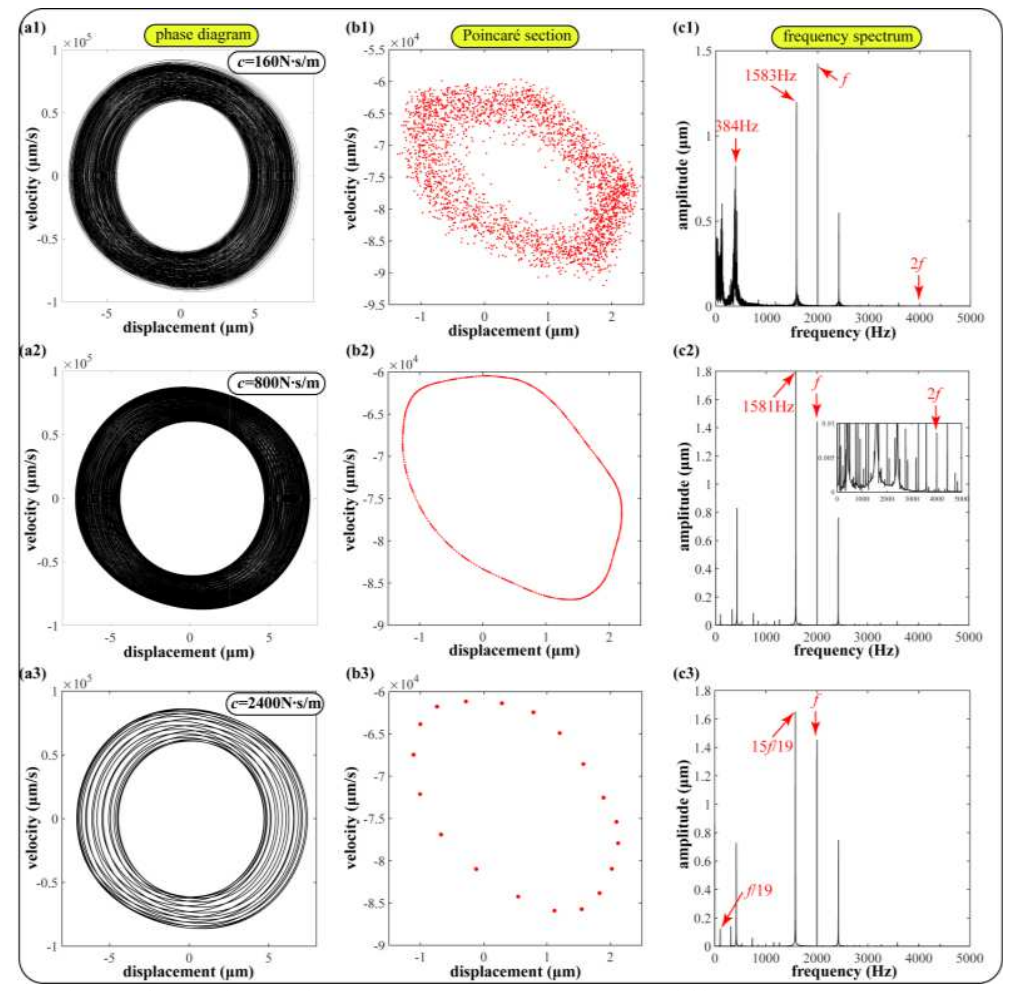

Fig. 27 The evolution of detailed vibration response under different damping of matched bearings when $f=2 \mathrm{kHz}, E=5000, L=800 \mathrm{~mm}, L_{p}=400 \mathrm{~mm}$, preloads of carriage, nut and bearing are $623 \mathrm{~N}$,

\section{Conclusion}

$1690 \mathrm{~N}$ and $290 \mathrm{~N}$.

This paper contributes a comprehensive dynamic model of BSFS to investigate the nonlinear behavior induced by nonlinear joint characteristics and coupling effect. The combined loads from six directions are applied on the worktable and the derived translational and angular deformations of each joint are calculated. The restoring force expression comprising coupling displacement of each joint is obtained. According to mass-spring-damping model, the analytical 18-DOF dynamic equation is established. Model verification tests are carried out and the results show a good match between simulation and measurement. Effect of external excitation, worktable position, screw length, preload and damping of joint on dynamic response is evaluated. Several conclusions can be drawn:

(1) The jumping phenomenon, super- and sub-harmonic solutions, soften and harden effects are captured by frequency response curve. The multiple resonance zones of nonlinear system are excited. The different main resonance frequencies among six directions are revealed, and the lowest frequency is in feed direction and the highest one is in pitching direction. Larger excitation amplitudes lead to higher peak-to-peak value of response and postpone the resonance frequency corresponding to jumping point.

(2) The identical evolution path of nonlinear behavior in six directions is discovered by bifurcation diagram. As the excitation amplitude increases, the unstable region is widened and jumping discontinuous behavior is triggered. The integral and non-integral multiples of primary frequency, as well as continuous frequency are captured in spectrum corresponding to quasi-period and chaotic motions.

(3) The lowest main resonance frequency point in feed direction appears in the middle position of screw. As worktable is close to two supported ends, the main resonance frequency increases symmetrically. The unstable frequency area is slightly expanded as the worktable is far from fixed end of matched bearings. Additionally, BSFS tends to lose the stability when worktable is located at the both end of screw.

(4) Larger preload and damping coefficient of rolling joints are beneficial for the stability of system since the unstable quasi-period and chaotic motions develop into single-period motion with the increased preload and damping.

Possible practical applications of the developed model can be concerned with optimizing structure, reducing the unstable motion state and vibration amplitude, improving accuracy of 
virtual prototype, etc.

\section{Acknowledgement}

The work is supported by National Natural Science Foundation of China (Grant No. 52075087, No. U1708254), the Fundamental Research Funds for the Central Universities (Grant No. N2003006, No. N2103003).

\section{Conflicts of interest}

There is no conflict of interest in the submission of this manuscript and the manuscript is approved by all the authors for publication.

\section{Availability of data and material}

All the experimental data used in this paper have been deposited into the Northeastern University Library and are publicly available for download.

\section{Code availability \\ Not applicable. \\ Authors' contributions}

Mengtao Xu: Methodology; Investigation; Experimental; Writing-Original Draft; WritingReview \& Editing

Changyou Li: Methodology; Resources and Supervision

Hongzhuang Zhang: Supervision; Writing-Review \& Editing

Zhendong Liu: Supervision; Writing-Review \& Editing

Yimin Zhang: Resources and Supervision

\section{Ethics approval}

Not applicable.

\section{Consent to participate}

Not applicable. The article involves no studies on humans.

\section{Consent for publication}

All authors have read and agreed to the published version of the manuscript.

\section{References}

1. Altintas Y, Verl A, Brecher C, Uriarte L, Pritschow G (2011) Machine tool feed drives. CIRP Ann-Manuf. Technol., 60 (2):779-796. doi:10.1016/j.cirp.2011.05.010

2. Brecher C, Eßer B, Falker J, Kneer F, Fey M (2018) Modelling of ball screw drives rolling element contact characteristics. CIRP Ann-Manuf. Technol., 67 (1):409-412. doi:10.1016/j.cirp.2018.04.109

3. Kamalzadeh A, Gordon DJ, Erkorkmaz K (2010) Robust compensation of elastic deformations in ball screw drives. Int. J. Mach. Tools Manuf., 50 (6):559-574. doi:10.1016/j.ijmachtools.2010.03.001

4. Huang H-W, Tsai M-S, Huang Y-C (2018) Modeling and elastic deformation compensation of flexural feed drive system. Int. J. Mach. Tools Manuf., 132:96-112. doi:10.1016/j.ijmachtools.2018.05.002

5. Hung J-P, Lai Y-L, Lin C-Y, Lo T-L (2011) Modeling the machining stability of a vertical milling machine under the influence of the preloaded linear guide. Int. J. Mach. Tools Manuf., 51 (9):731-739. doi:10.1016/j.ijmachtools.2011.05.002

6. Liang T, Lu D, Yang X, Zhang J, Ma X, Zhao W (2016) Feed fluctuation of ball screw feed systems and its effects on part surface quality. Int. J. Mach. Tools Manuf., 101:1-9. doi:10.1016/j.ijmachtools.2015.11.002

7. Zhang X, Zhang J, Zhang W, Liang T, Liu H, Zhao W (2018) Integrated modeling and analysis of ball screw feed system and milling process with consideration of multi-excitation effect. Mech. Syst. Signal Proc., 98:484-505. doi:10.1016/j.ymssp.2017.05.011

8. Feng G-H, Pan Y-L (2012) Investigation of ball screw preload variation based on dynamic modeling of a preload adjustable feed-drive system and spectrum analysis of ball-nuts sensed 
vibration signals. Int. J. Mach. Tools Manuf., 52 (1):85-96. doi:10.1016/j.ijmachtools.2011.09.008 9. Zhang GP, Huang YM, Shi WH, Fu WP (2003) Predicting dynamic behaviours of a whole machine tool structure based on computer-aided engineering. Int. J. Mach. Tools Manuf., 43 (7):699-706. doi:10.1016/s0890-6955(03)00026-9

10. Li B, Luo B, Mao X, Cai H, Peng F, Liu H (2013) A new approach to identifying the dynamic behavior of CNC machine tools with respect to different worktable feed speeds. Int. J. Mach. Tools Manuf., 72:73-84. doi:10.1016/j.ijmachtools.2013.06.004

11. Zhang J, Zhang H, Du C, Zhao W (2016) Research on the dynamics of ball screw feed system with high acceleration. Int. J. Mach. Tools Manuf., 111:9-16. doi:10.1016/j.ijmachtools.2016.09.001

12. Wang W, Li C, Zhou Y, Wang H, Zhang Y (2018) Nonlinear dynamic analysis for machine tool table system mounted on linear guides. Nonlinear Dyn., 94 (3):2033-2045. doi:10.1007/s11071-018-4473-x

13. Wang W, Zhou Y, Wang H, Li C, Zhang Y (2019) Vibration analysis of a coupled feed system with nonlinear kinematic joints. Mech. Mach. Theory, 134:562-581. doi:10.1016/j.mechmachtheory.2019.01.014

14. Yang T, Hou S, Qin Z-H, Ding Q, Chen L-Q (2021) A dynamic reconfigurable nonlinear energy sink. J. Sound Vibr., 494:115629. doi:10.1016/j.jsv.2020.115629

15. Altintas Y, Brecher C, Weck M, Witt S (2005) Virtual Machine Tool. CIRP Ann-Manuf. Technol., 54 (2):115-138. doi:10.1016/s0007-8506(07)60022-5

16. Vicente DA, Hecker RL, Villegas FJ, Flores GM (2011) Modeling and vibration mode analysis of a ball screw drive. Int. J. Adv. Manuf. Technol., 58 (1-4):257-265. doi:10.1007/s00170-011-3375-6

17. Dong L, Tang WC (2014) Adaptive backstepping sliding mode control of flexible ball screw drives with time-varying parametric uncertainties and disturbances. ISA Trans., 53 (1):110-116. doi:10.1016/j.isatra.2013.08.009

18. Wu Q, Gu F, Ball A, Huang H (2021) Hybrid model for the analysis of the modal properties of a ball screw vibration system. J. Mech. Sci. Technol., 35 (2):461-470. doi:10.1007/s12206-021-0104-4

19. Frey S, Dadalau A, Verl A (2012) Expedient modeling of ball screw feed drives. Production Engineering 6 (2):205-211. doi:10.1007/s11740-012-0371-0

20. Sato R (2019) Sensor-less estimation of positioning reversal value for ball screw feed drives. Precis. Eng.-J. Int. Soc. Precis. Eng. Nanotechnol., 60:116-120. doi:10.1016/j.precisioneng.2019.06.011

21. Ansoategui I, Campa FJ (2017) Mechatronics of a ball screw drive using an $\mathrm{N}$ degrees of freedom dynamic model. Int. J. Adv. Manuf. Technol., 93 (1-4):1307-1318. doi:10.1007/s00170-017-0597-2

22. Guo C, Chen L, Ding J (2019) A novel dynamics model of ball-screw feed drives based on theoretical derivations and deep learning. Mech. Mach. Theory, 141:196-212. doi:10.1016/j.mechmachtheory.2019.07.011

23. Nguyen TL, Ro S-K, Park J-K (2019) Study of ball screw system preload monitoring during operation based on the motor current and screw-nut vibration. Mech. Syst. Signal Proc., 131:18-32. doi:10.1016/j.ymssp.2019.05.036

24. Wang L, Liu H, Yang L, Zhang J, Zhao W, Lu B (2015) The effect of axis coupling on machine 
tool dynamics determined by tool deviation. Int. J. Mach. Tools Manuf., 88:71-81. doi:10.1016/j.ijmachtools.2014.09.003

25. Zou C, Zhang H, Lu D, Zhang J, Zhao W (2017) Effect of the screw-nut joint stiffness on the position-dependent dynamics of a vertical ball screw feed system without counterweight. Proceedings of the Institution of Mechanical Engineers, Proc. Inst. Mech. Eng. Part C-J. Eng. Mech. Eng. Sci., 232 (15):2599-2609. doi:10.1177/0954406217722378

26. Zhang H, Liu H, Du C, lv D, Zhang J, Zhao W (2018) Dynamics analysis of a slender ball-screw feed system considering the changes of the worktable position. Proc. Inst. Mech. Eng. Part C-J. Eng. Mech. Eng. Sci., 233 (8):2685-2695. doi:10.1177/0954406218799781

27. Zhang W, Zhang X, Zhang J, Zhao W (2019) Analysis of lead screw pre-stretching influences on the natural frequency of ball screw feed system. Precis. Eng.-J. Int. Soc. Precis. Eng. Nanotechnol., 57:30-44. doi:10.1016/j.precisioneng.2019.03.003

28. Li C, Xu M, He G, Zhang H, Liu Z, He D, Zhang Y (2020) Time-dependent nonlinear dynamic model for linear guideway with crowning. Tribol. Int., 151:106413. doi:10.1016/j.triboint.2020.106413

29. Xu M, Zhang H, Liu Z, Li C, Zhang Y, Mu Y, Hou C (2021) A time-dependent dynamic model for ball passage vibration analysis of recirculation ball screw mechanism. Mech. Syst. Signal Proc., 157:107632. doi:10.1016/j.ymssp.2021.107632

30. Gu J, Zhang Y (2019) Dynamic analysis of a ball screw feed system with time-varying and piecewise-nonlinear stiffness. Proc. Inst. Mech. Eng. Part C-J. Eng. Mech. Eng. Sci., 233(18):6503-6518. doi:10.1177/0954406219865923

31. Xu M, Cai B, Li C, Zhang H, Liu Z, He D, Zhang Y (2020) Dynamic characteristics and reliability analysis of ball screw feed system on a lathe. Mech. Mach. Theory, 150:103890. doi:10.1016/j.mechmachtheory.2020.103890

32. Kong X, Sun W, Wang B, Wen B (2015) Dynamic and stability analysis of the linear guide with time-varying, piecewise-nonlinear stiffness by multi-term incremental harmonic balance method. J. Sound Vibr., 346:265-283. doi:10.1016/j.jsv.2015.02.021

33. Liu Z, Xu M, Zhang H, Miao H, Li Z, Li C, Zhang Y (2021) Nonlinear dynamic analysis of ball screw feed system considering assembly error under harmonic excitation. Mech. Syst. Signal Proc., 157:107717. doi:10.1016/j.ymssp.2021.107717

34. Liu J, Shao Y (2017) Dynamic modeling for rigid rotor bearing systems with a localized defect considering additional deformations at the sharp edges. J. Sound Vibr., 398:84-102. doi:10.1016/j.jsv.2017.03.007

35. Zhang X, Han Q, Peng Z, Chu F (2015) A new nonlinear dynamic model of the rotor-bearing system considering preload and varying contact angle of the bearing. Commun. Nonlinear Sci. Numer. Simul., 22 (1-3):821-841. doi:10.1016/j.cnsns.2014.07.024

36. Bai C, Zhang H, Xu Q (2007) Effects of axial preload of ball bearing on the nonlinear dynamic characteristics of a rotor-bearing system. Nonlinear Dyn., 53 (3):173-190. doi:10.1007/s11071-007-9306-2

37. Wang Z, Zhu C (2020) A new model for analyzing the vibration behaviors of rotor-bearing system. Commun. Nonlinear Sci. Numer. Simul., 83:105130. doi:10.1016/j.cnsns.2019.105130

38. Sun W, Kong X, Wang B, Li X (2014) Statics modeling and analysis of linear rolling guideway considering rolling balls contact. Proc. Inst. Mech. Eng. Part C-J. Eng. Mech. Eng. Sci., 229 (1):168-179. doi:10.1177/0954406214531943 
39. Harris TA (2001) Rolling bearing analysis, fourth ed. New York: John Wiley \& Sons.

40. Wei CC, Lin JF (2003) Kinematic analysis of the ball screw mechanism considering variable contact angles and elastic deformations. J. Mech. Des.-Trans. ASME, 125 (4):717-733

41. Gunduz A, Dreyer JT, Singh R (2012) Effect of bearing preloads on the modal characteristics of a shaft-bearing assembly: Experiments on double row angular contact ball bearings. Mech. Syst. Signal Proc., 31:176-195. doi:10.1016/j.ymssp.2012.03.013

42. Sopanen J, Mikkola A (2003) Dynamic model of a deep-groove ball bearing including localized and distributed defects. Part 1: Theory. Proc. Inst. Mech Eng Pt K-J Multi-Body Dyn., 217 (3):201-211. doi:10.1243/14644190360713551

43. Yu Y, Yao G, Wu Z (2020) Nonlinear primary responses of a bilateral supported X-shape vibration reduction structure. Mech. Syst. Signal Proc., 140:106679. doi:10.1016/j.ymssp.2020.106679

44. Li S, Wu Q, Zhang Z (2013) Bifurcation and chaos analysis of multistage planetary gear train. Nonlinear Dyn., 75 (1-2):217-233. doi:10.1007/s11071-013-1060-z 


\section{Figures}

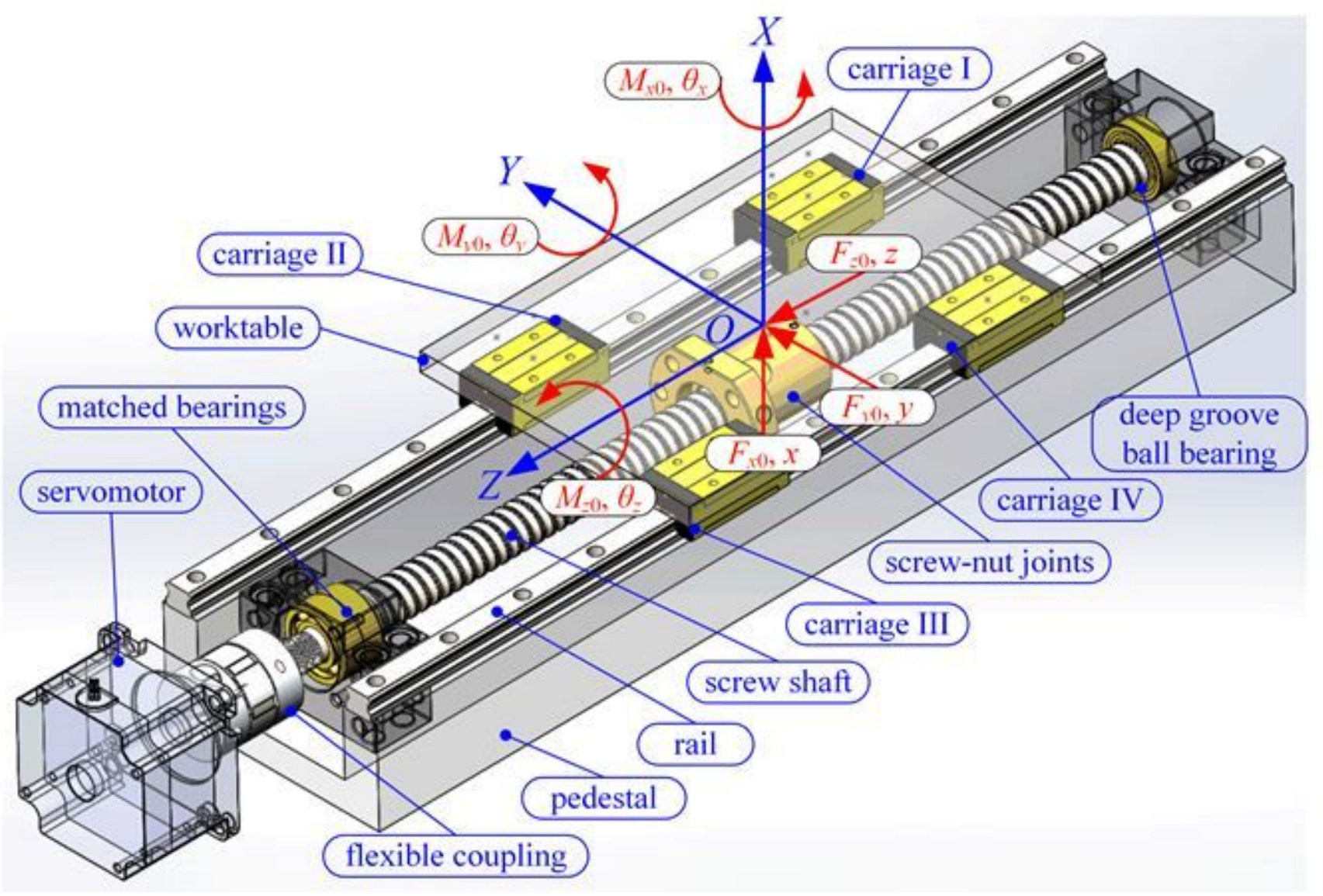

Figure 1

Schematic diagram of ball screw driven CNC axis 


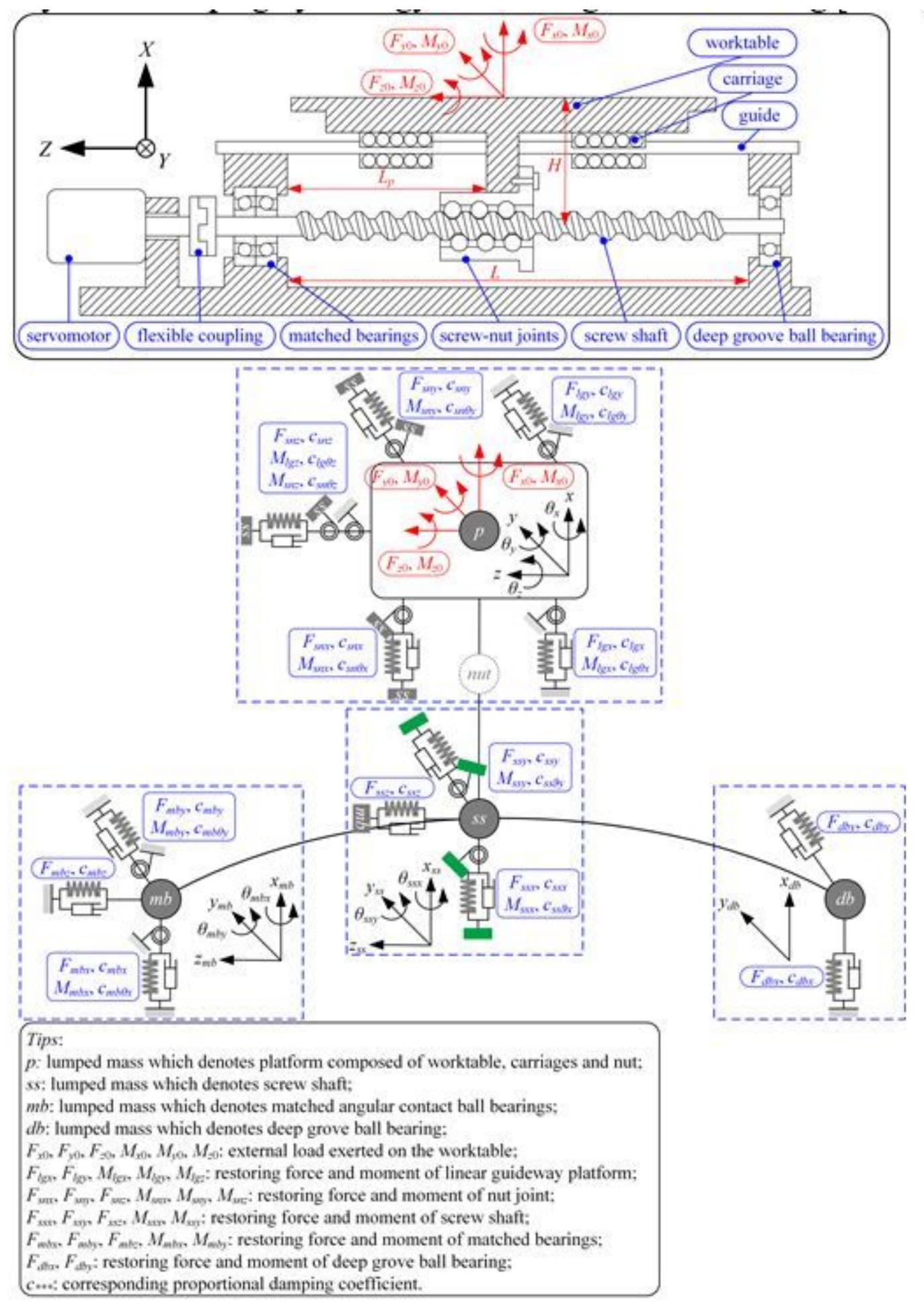

Figure 2

Equivalent dynamic model of ball screw feed system 

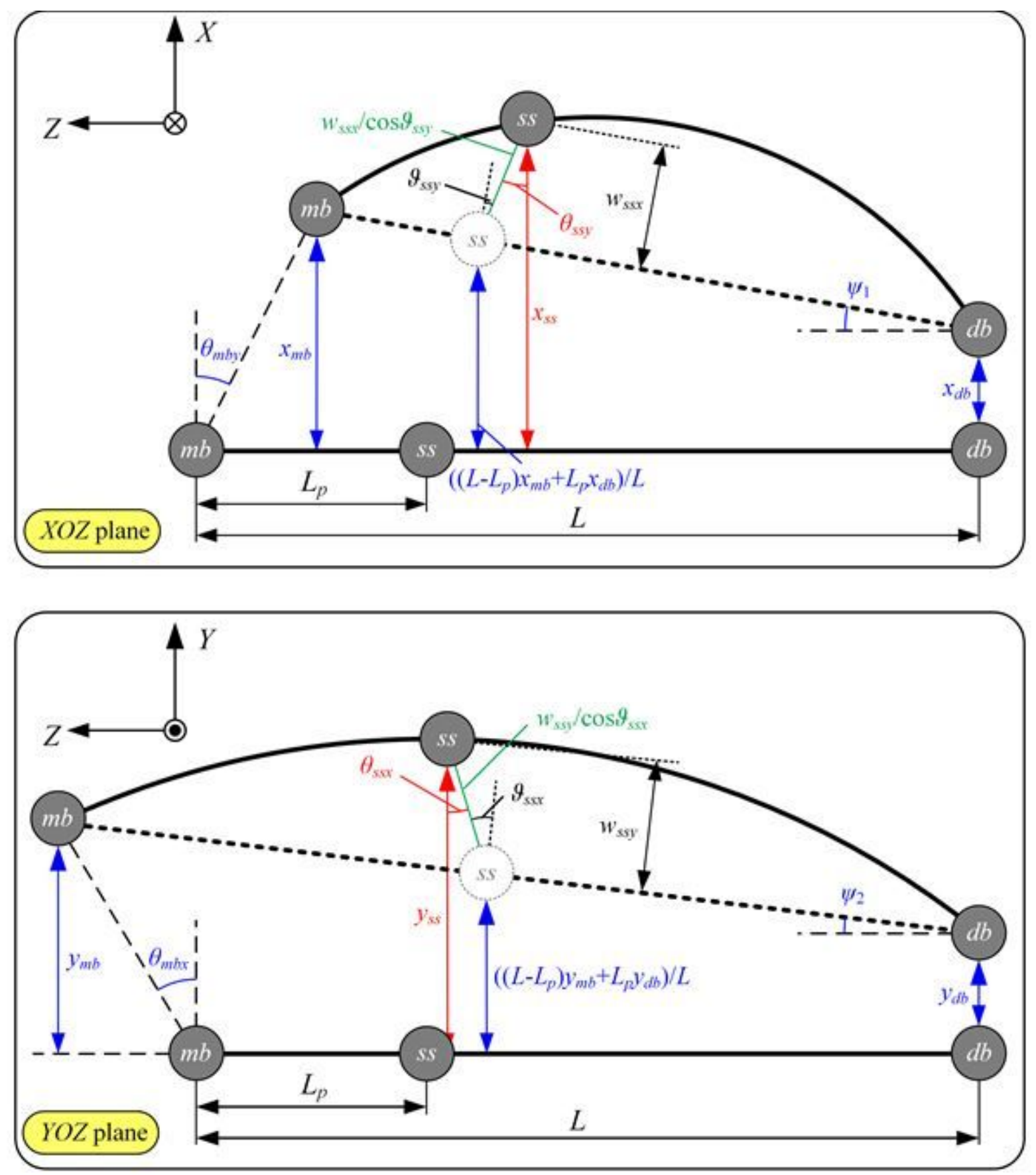

Figure 3

The relationship between absolute displacement and deformation of lumped screw shaft unit 


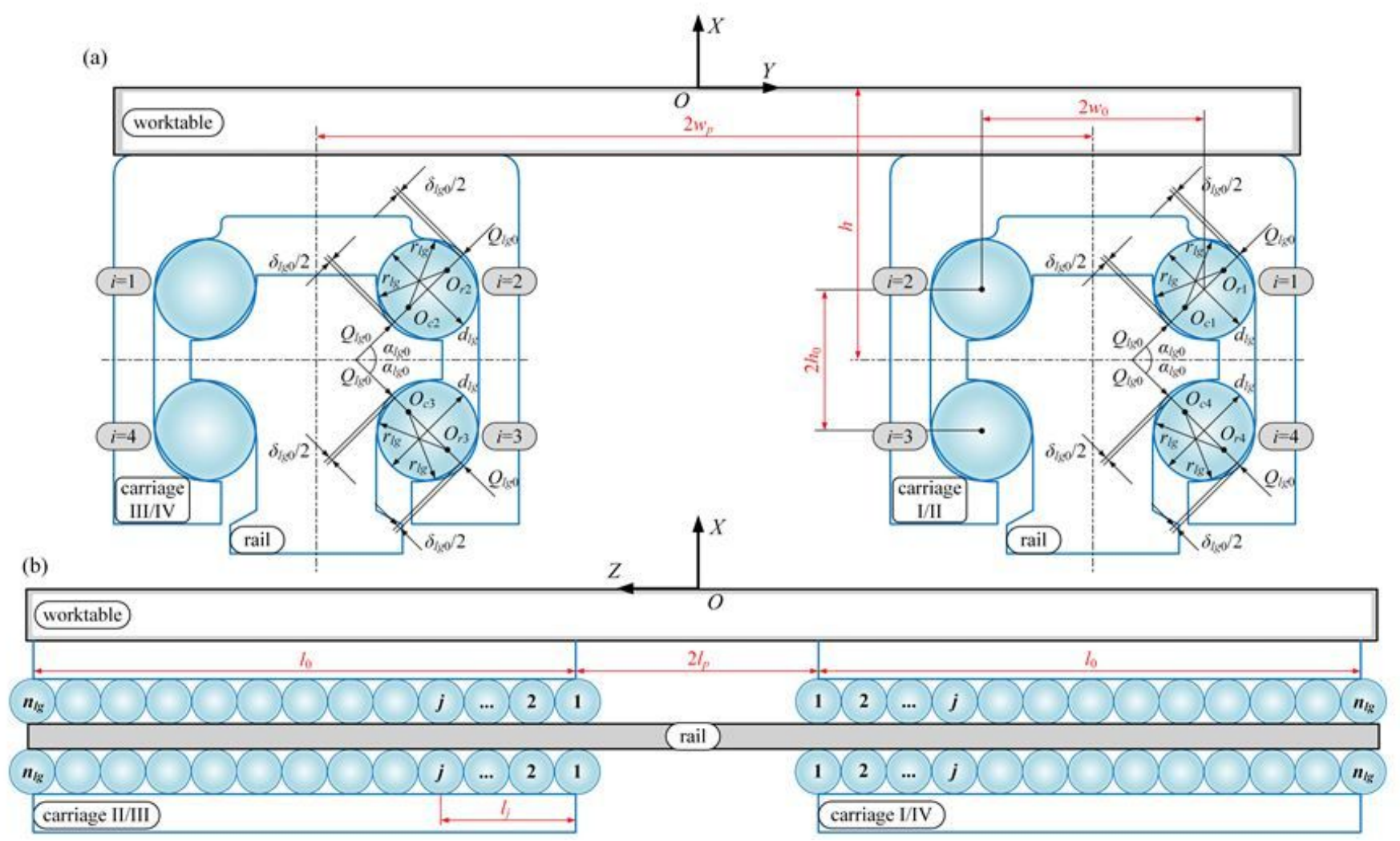

Figure 4

Ball arrangement within carriage; (a) ball contact geometry with face-to-face configuration; (b) ball location along feed direction 

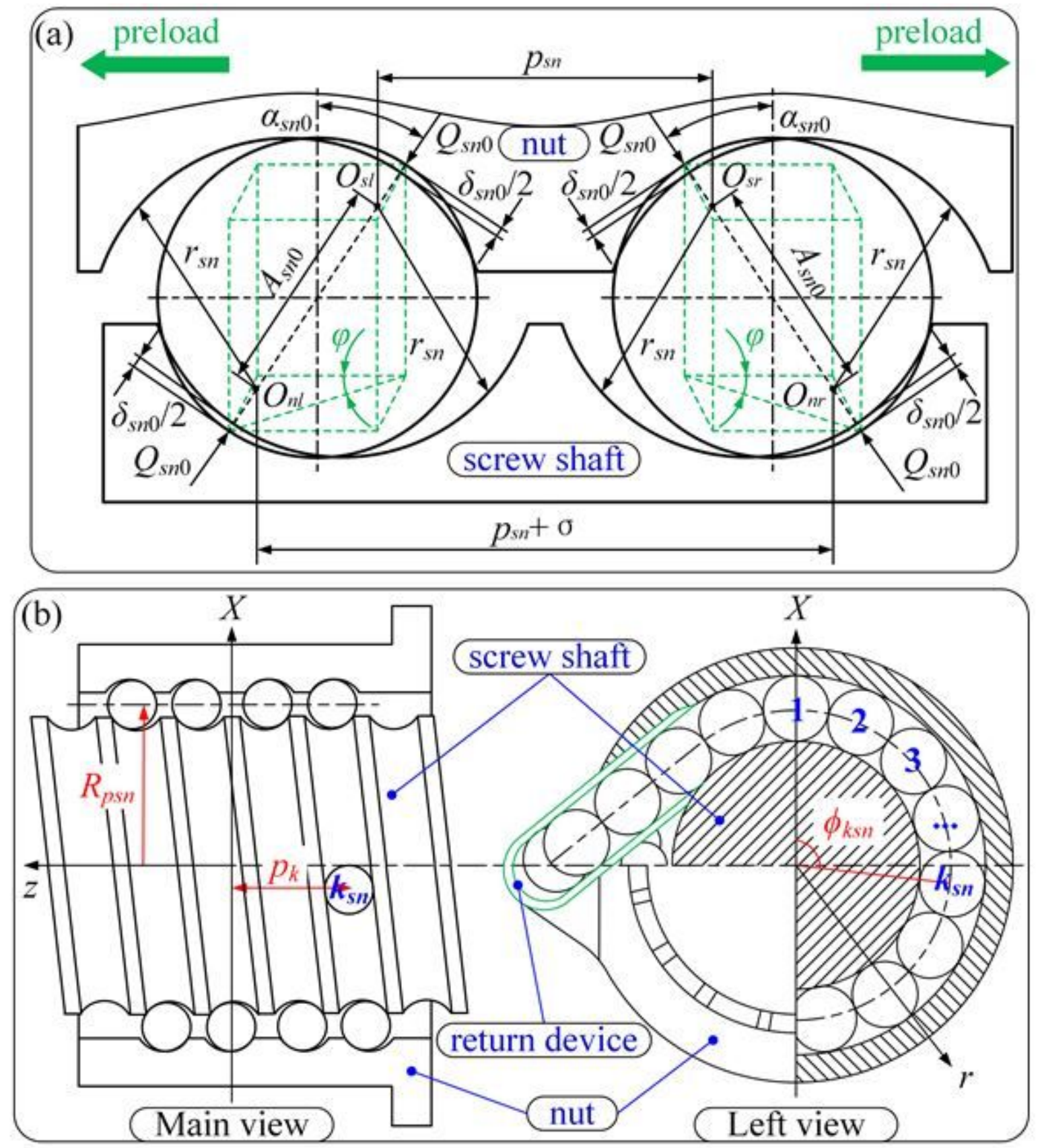

Figure 5

Ball arrangement within screw-nut joint; (a) ball contact geometry; (b) ball location 

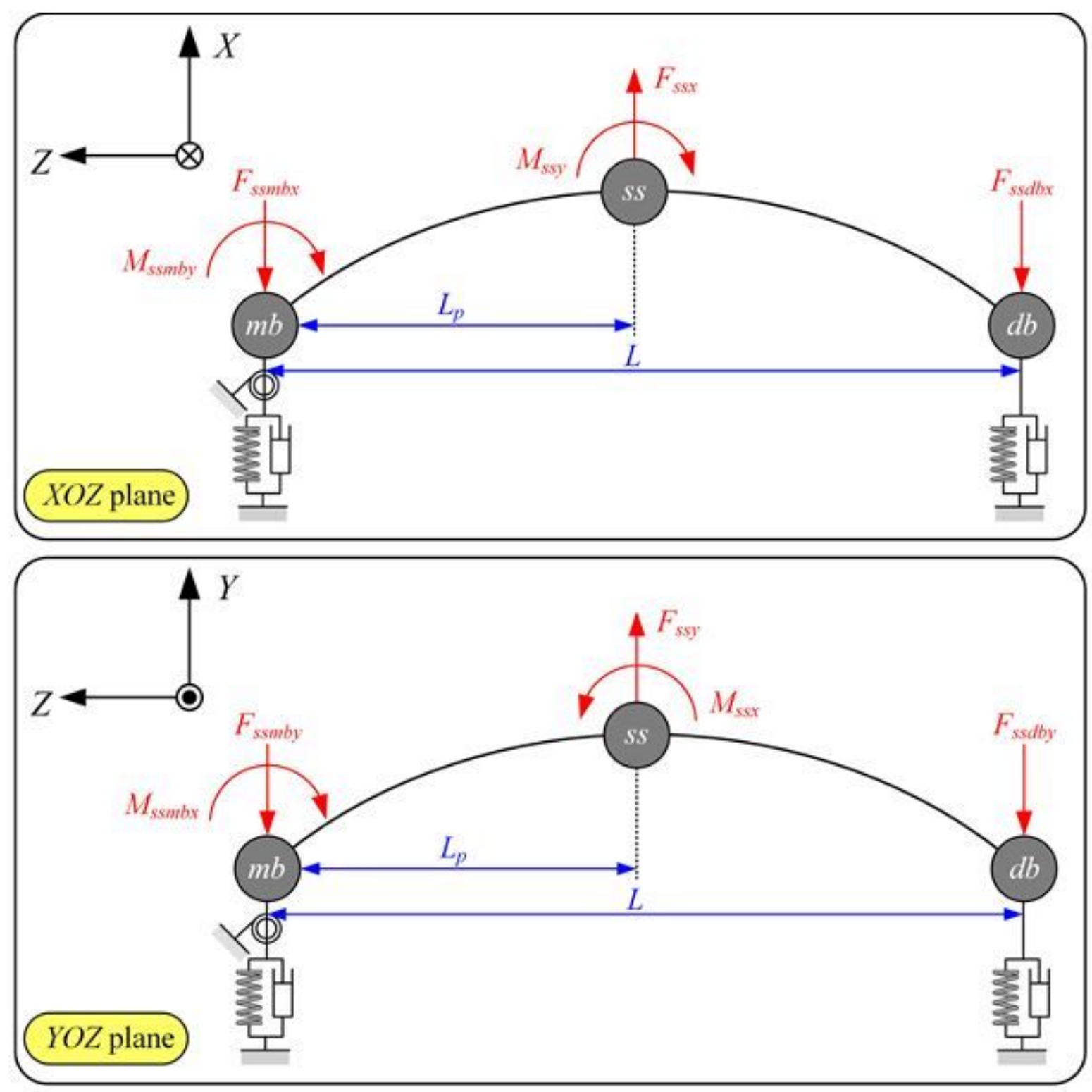

Figure 6

Load analysis of screw shaft unit 
(a)

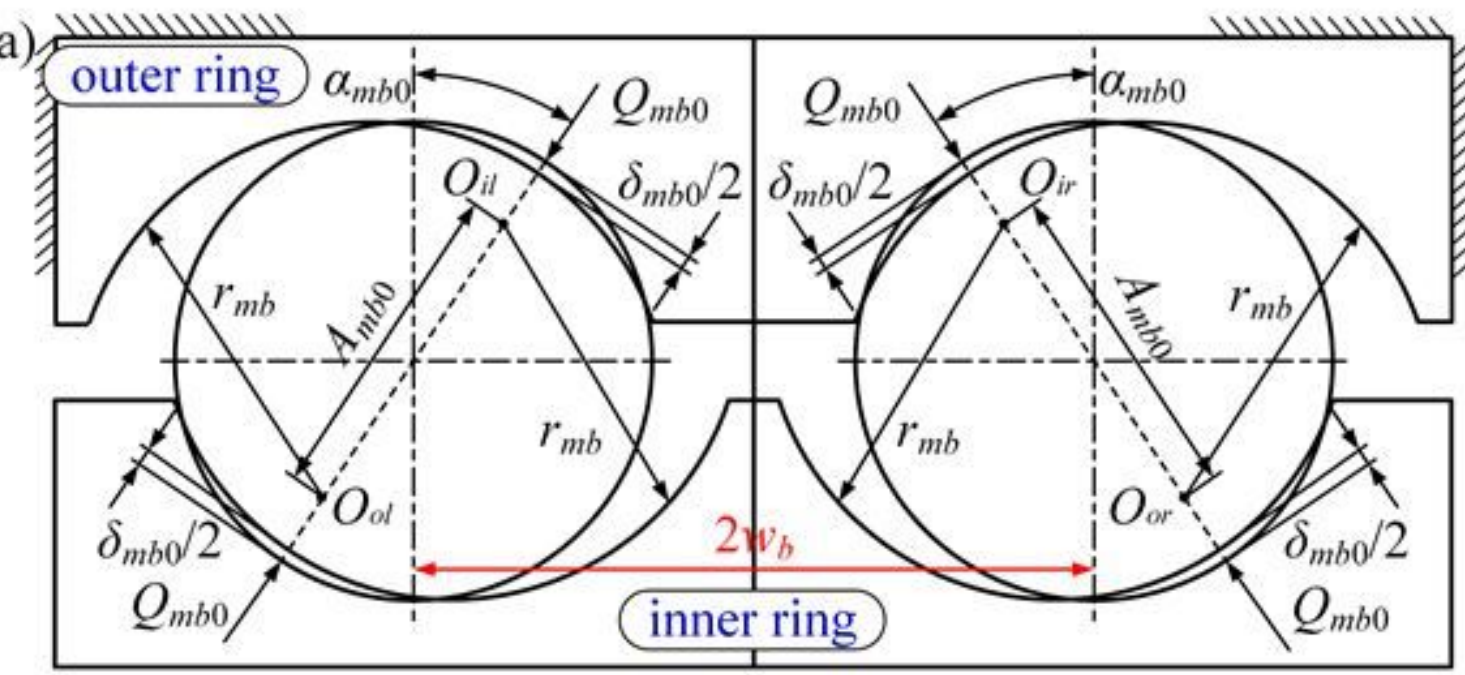

(b)

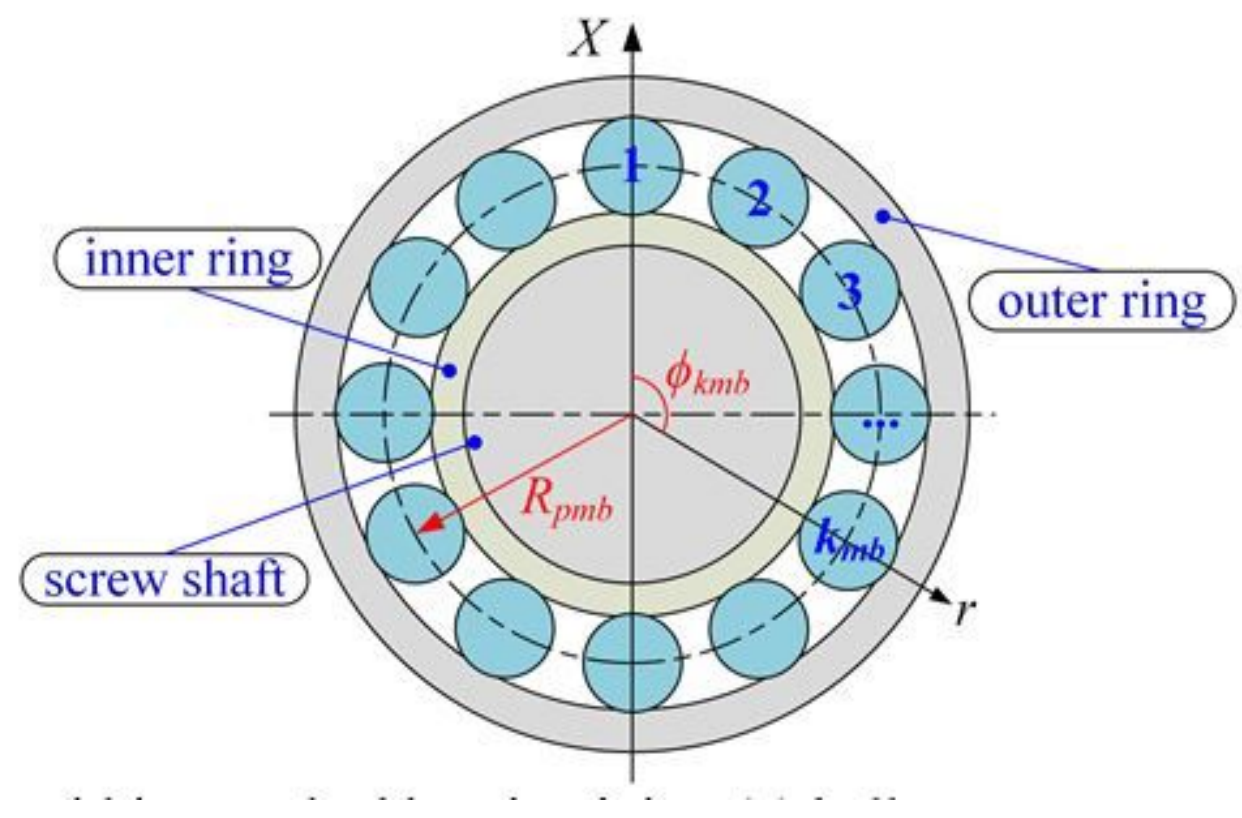

Figure 7

Ball arrangement within matched bearing joint; (a) ball contact geometry; (b) ball location 

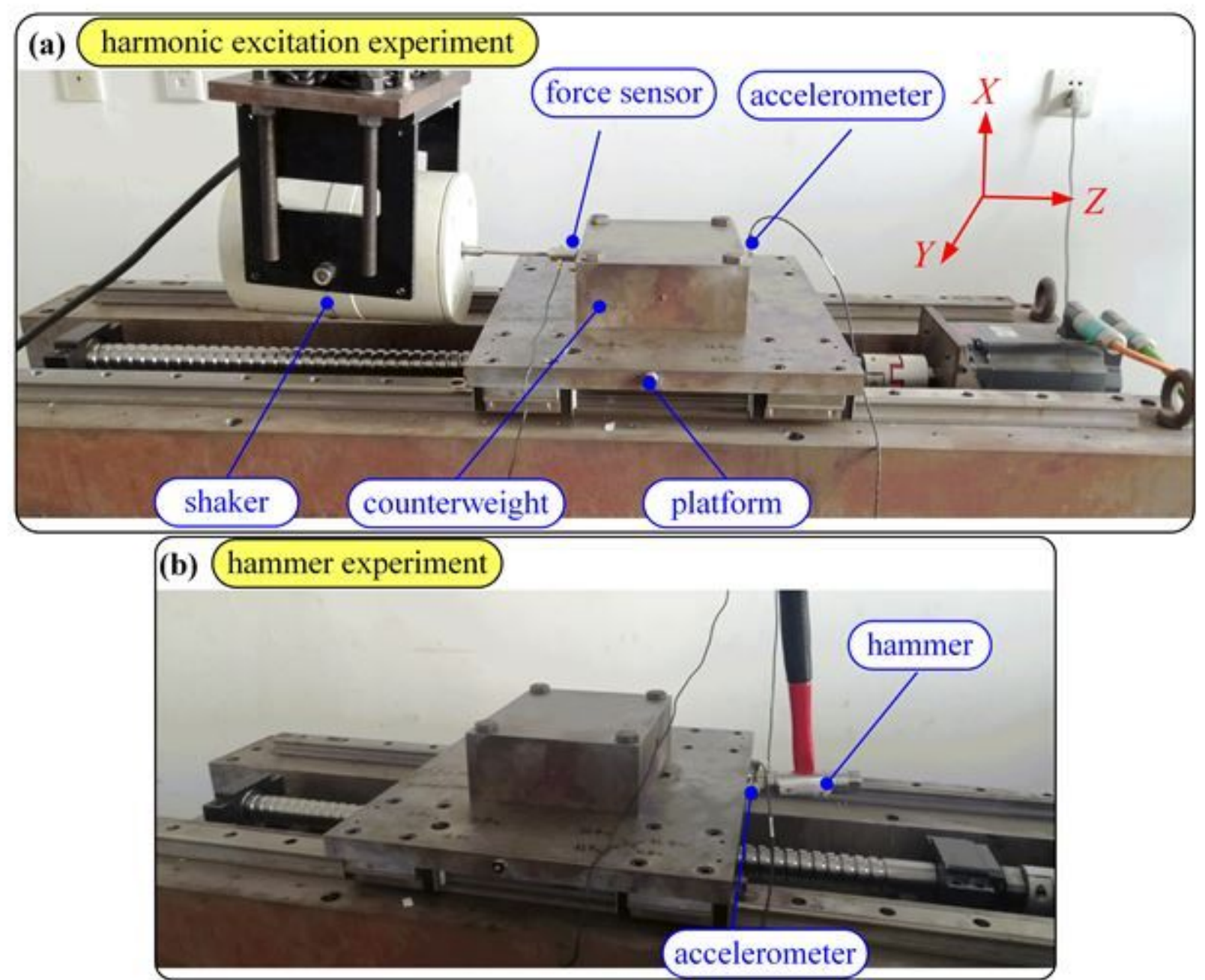

\section{Figure 8}

Dynamics measurement; (a) experimental setup for harmonic excitation; (b) impact testing 

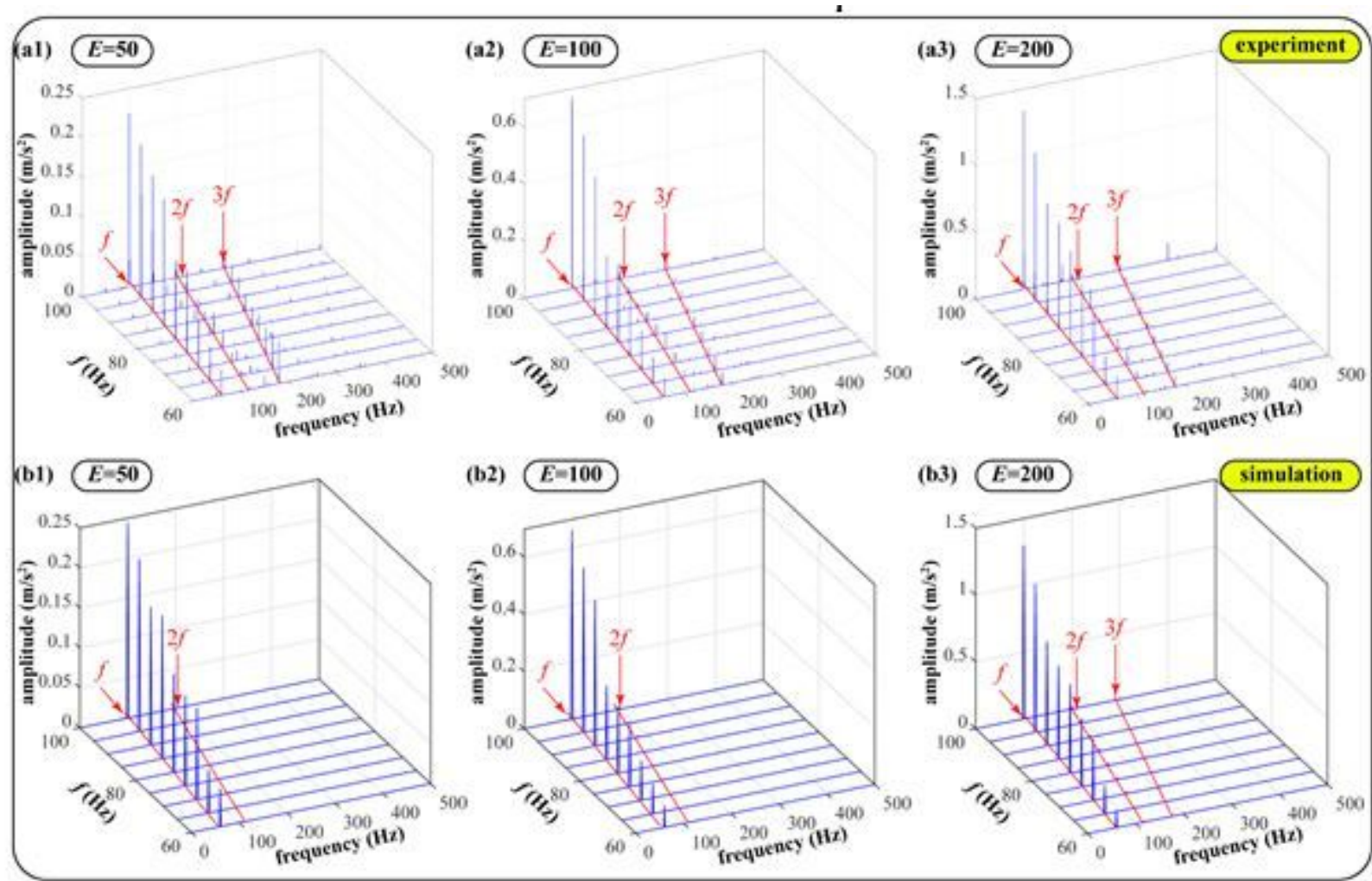

\section{Figure 9}

3-D frequency spectrum comparison between experimental and theoretical results; (a1-a3) experiment when external amplitudes are 50, 100, 200; (b1-b3) simulation when external amplitudes are 50, 100, 200
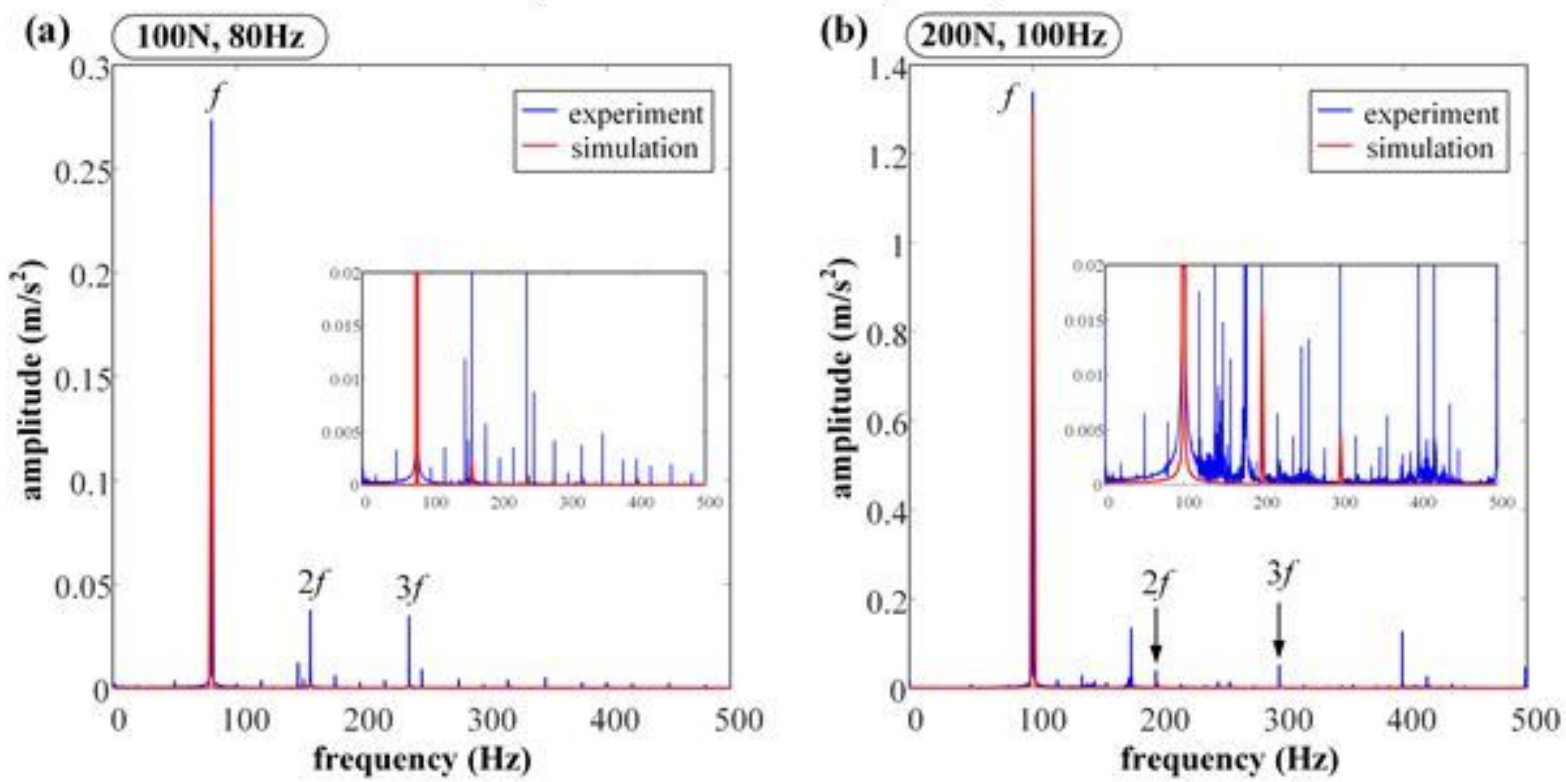

Figure 10

Detailed spectrum comparison between experimental and theoretical results; (a) excitation with $100 \mathrm{~N}$, $80 \mathrm{~Hz}$; (a) excitation with $200 \mathrm{~N}, 100 \mathrm{~Hz}$ 

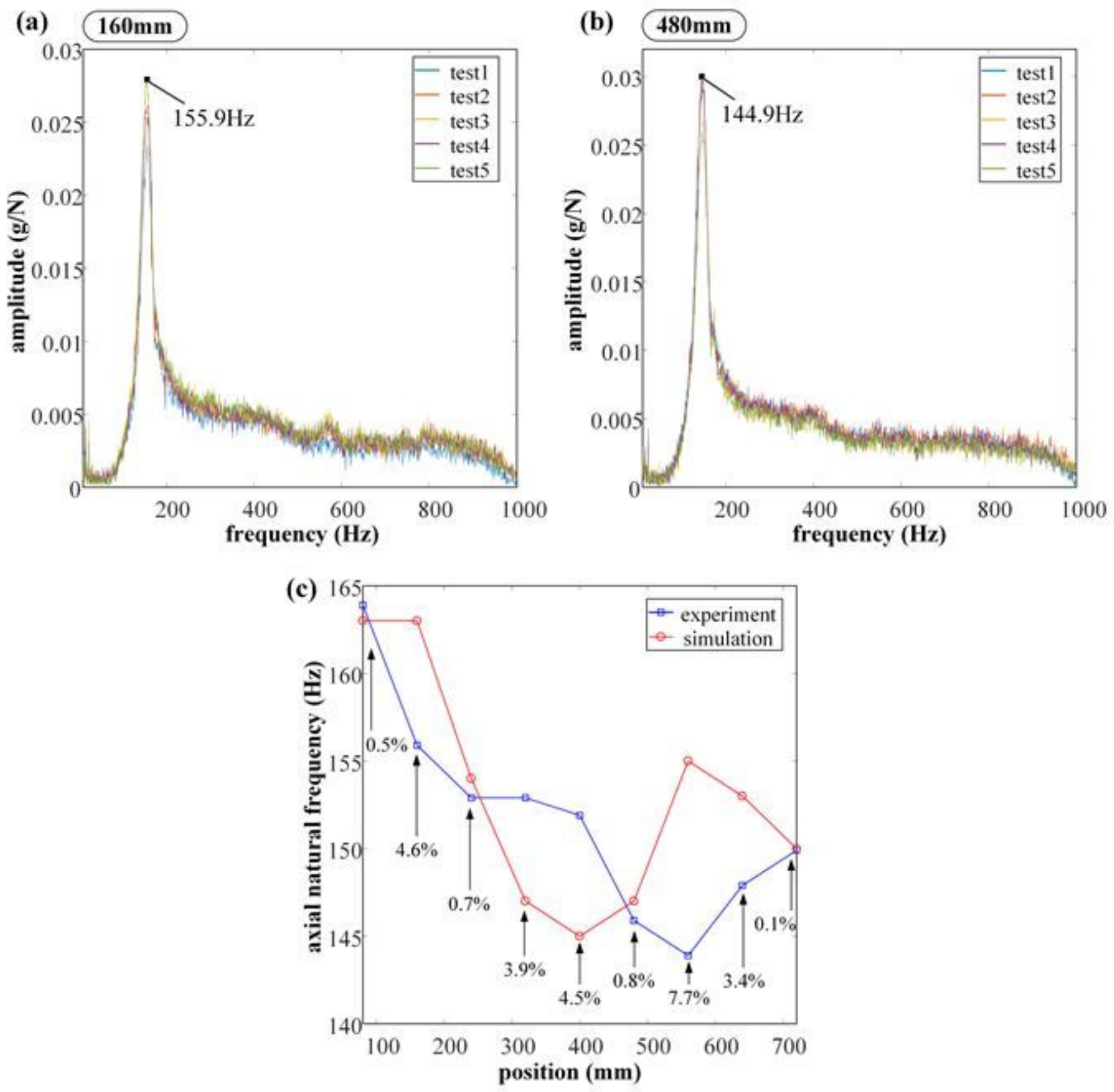

\section{Figure 11}

The results of impact test; (a-b) FRF when platform is loacted at $160 \mathrm{~mm}$ and $480 \mathrm{~mm}$; (c) axial natural frequency comparison between mesured and simulated results 

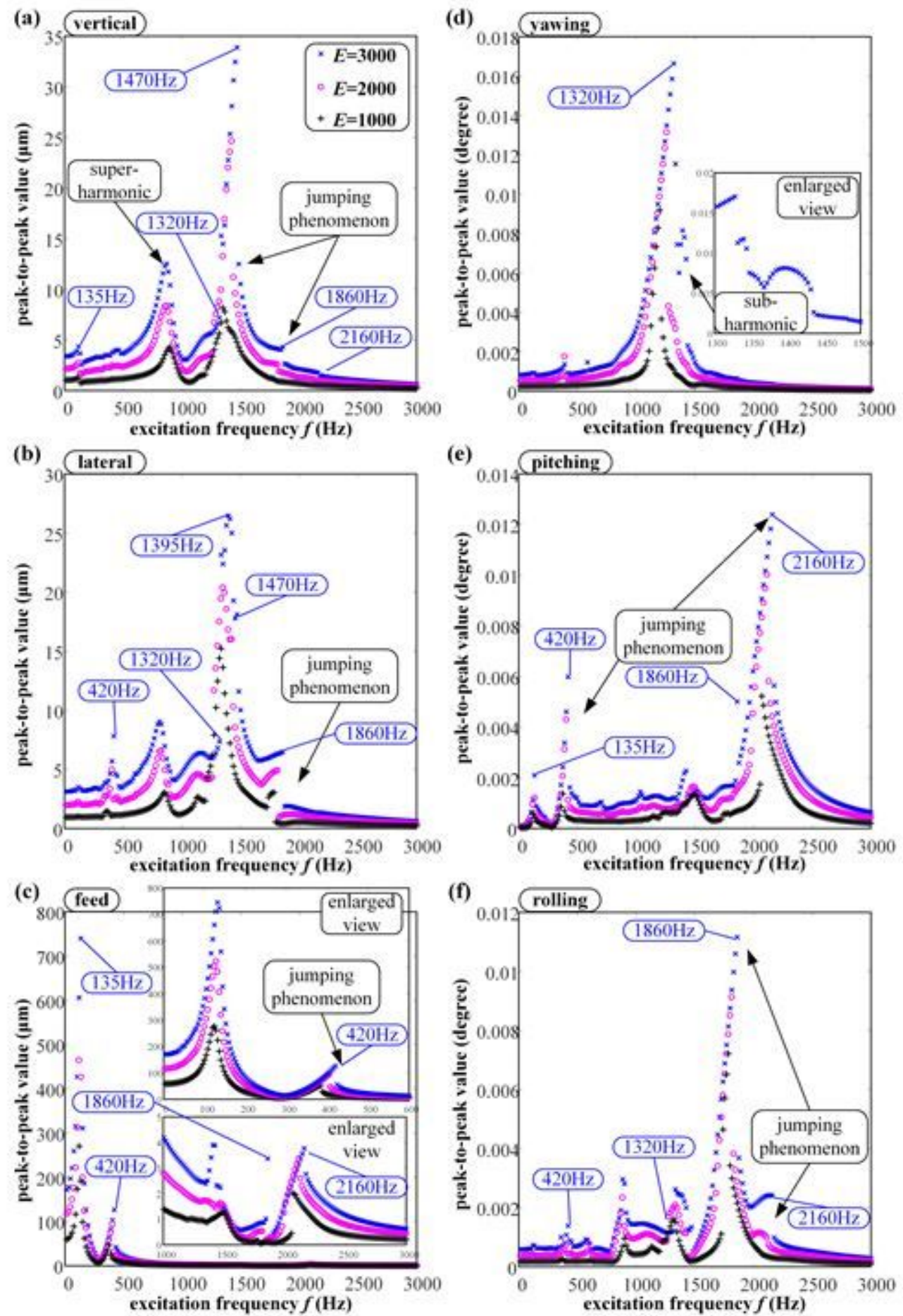

Figure 12

Frequency sweep plot of six vibration directions under different external load amplitudes 
(a) vertical

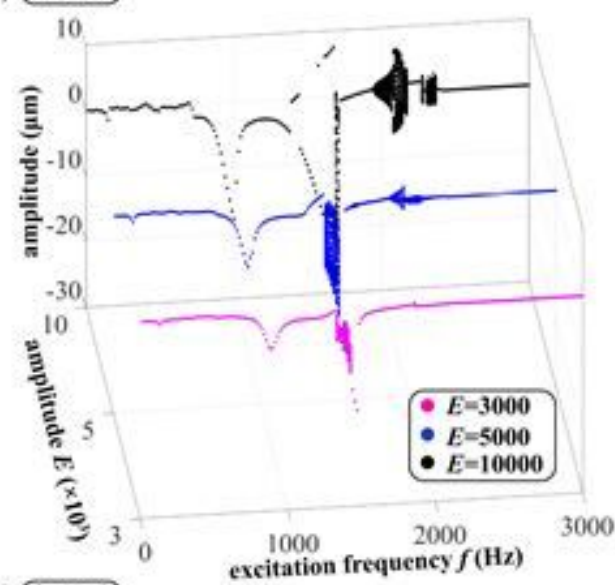

(b) lateral

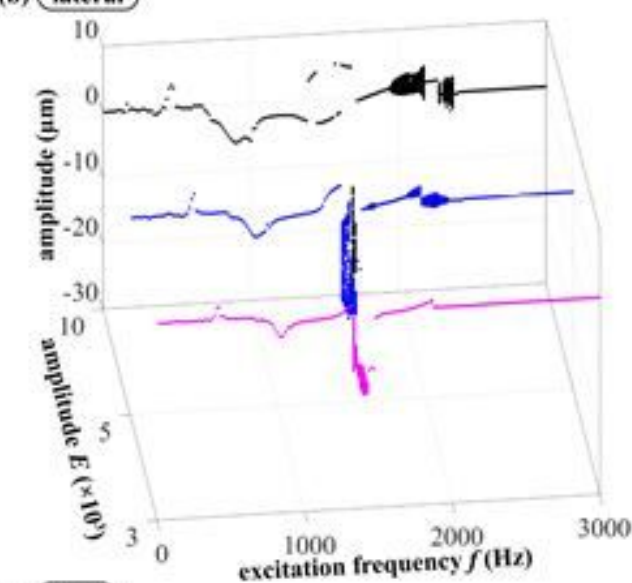

(c) feed

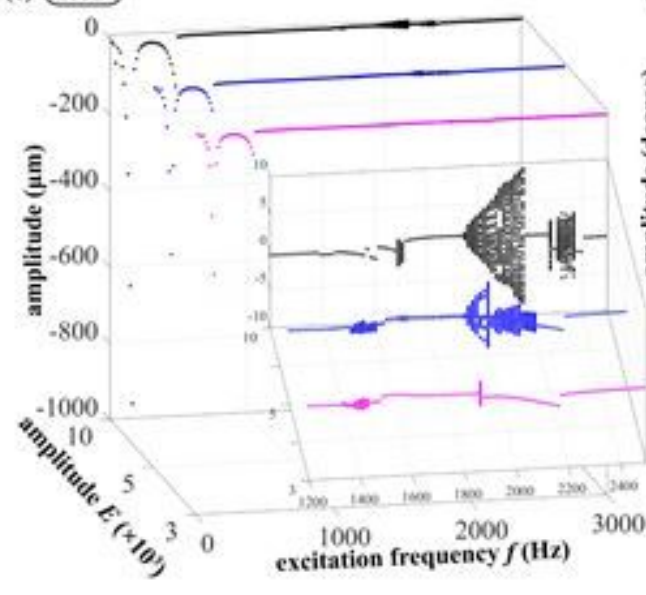

(d) yawing

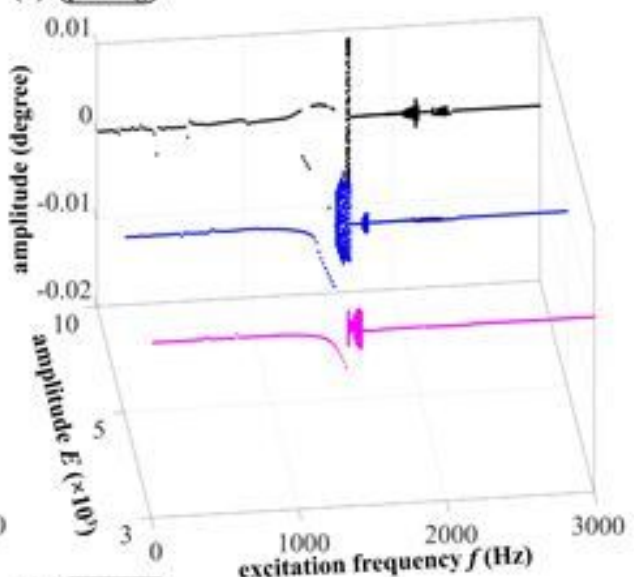

(e) pitching

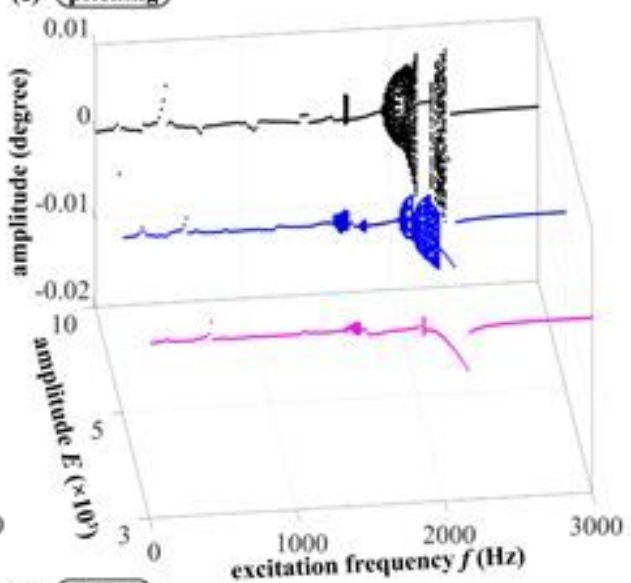

(f) rolling

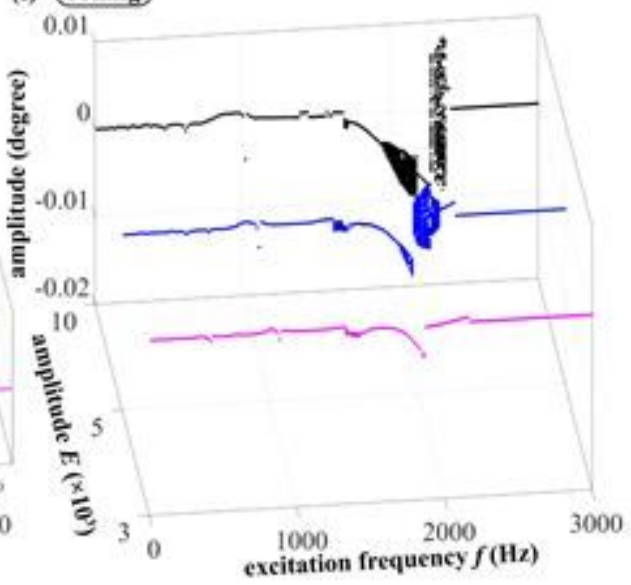

\section{Figure 13}

Bifurcation diagram of six vibration directions with excitation frequency as the control parameter under different excitation amplitude 

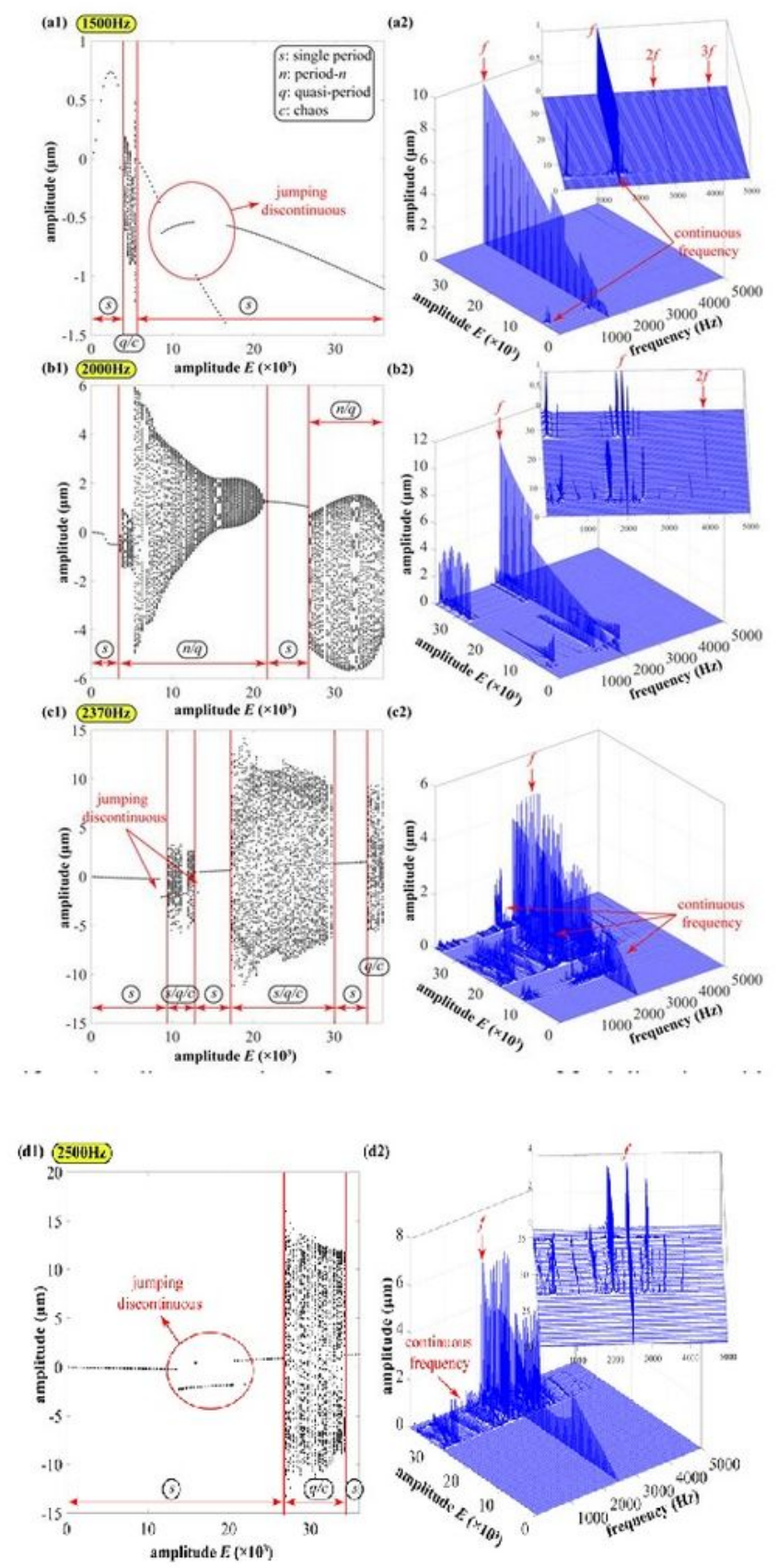

\section{Figure 14}

Bifurcation diagram and 3-D frequency spectrum of feed direction with excitation amplitude as the control parameter under different excitation frequency 

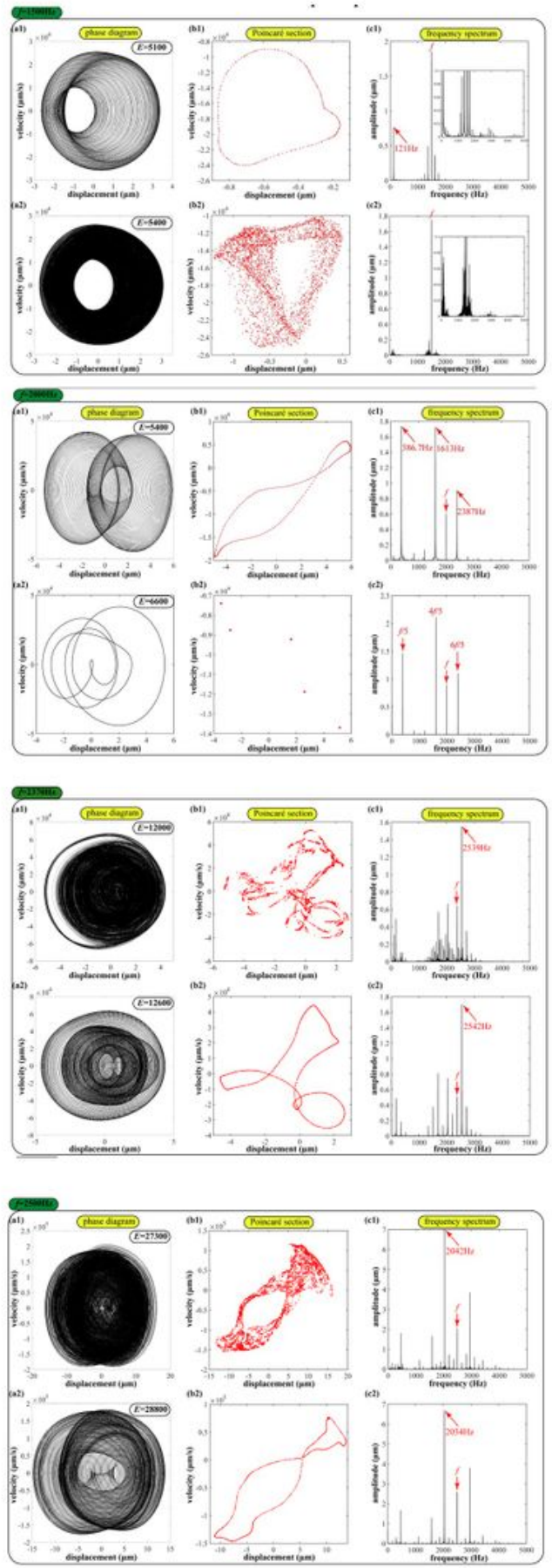

Figure 15

Vibration response under certain excitation frequency and amplitude 
(a1) $100 \mathrm{~mm}$

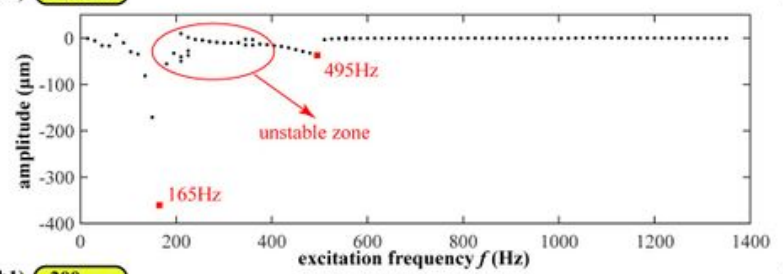

(b1) $200 \mathrm{~mm}$

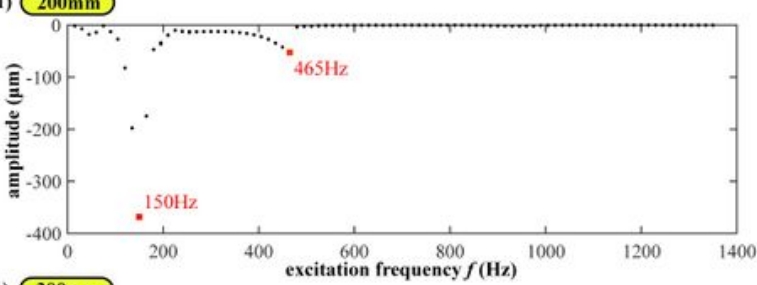

(c1) $300 \mathrm{~mm}$

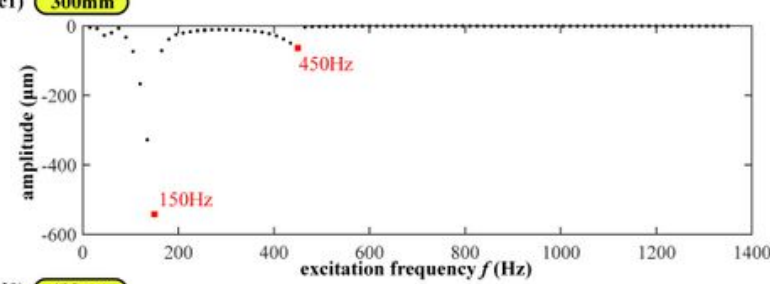

(d1) $400 \mathrm{~mm}$

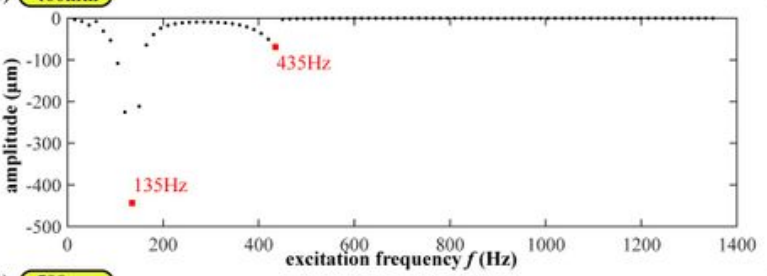

(e1) $500 \mathrm{~mm}$

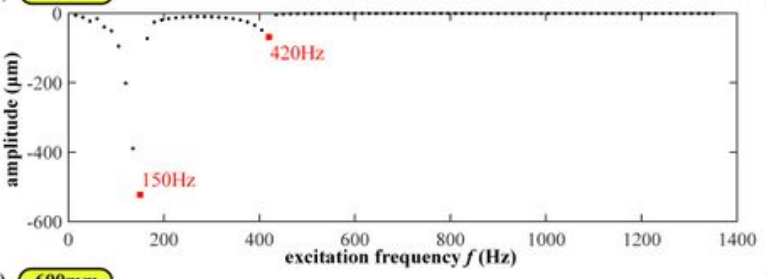

(fi) $600 \mathrm{~mm}$

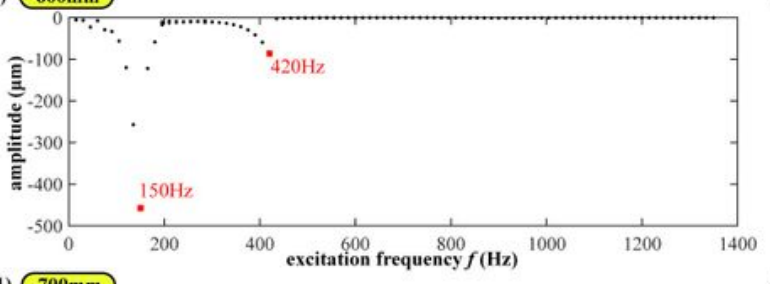

(g1) $700 \mathrm{~mm}$

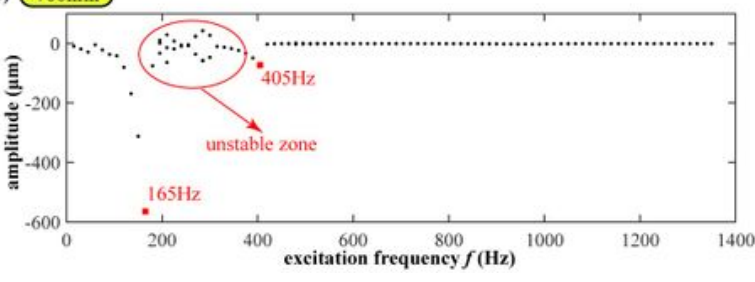

(a2) $100 \mathrm{~mm}$
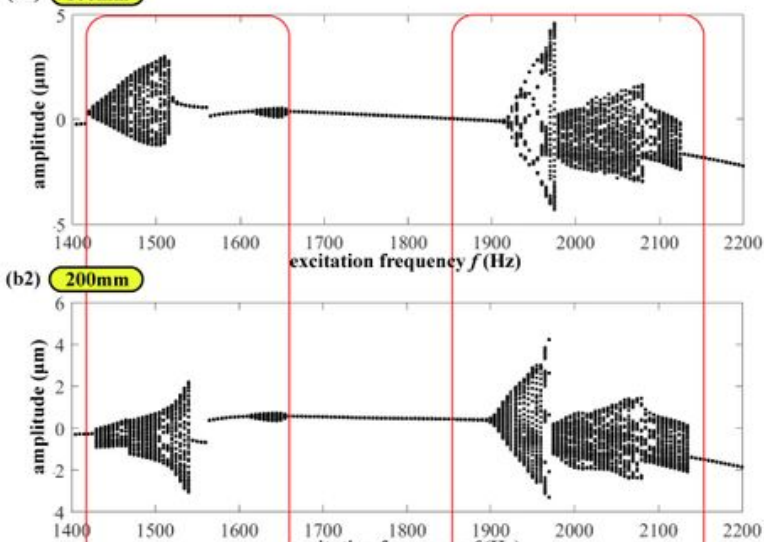

(c2) $300 \mathrm{~mm}$

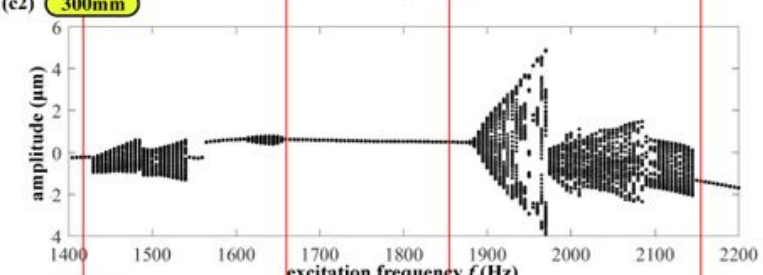

(d2) $400 \mathrm{~mm}$

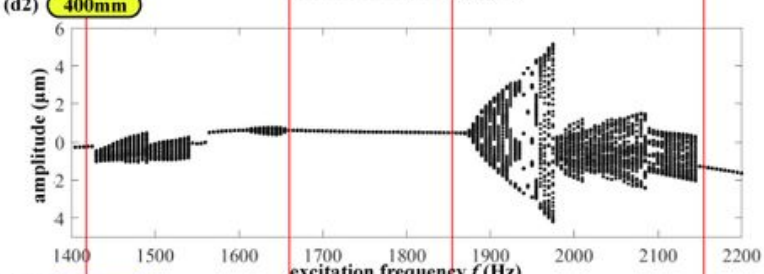

(e2) $500 \mathrm{~mm}$

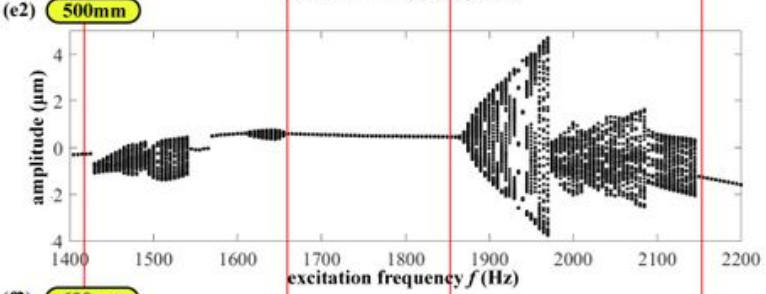

(f2) $600 \mathrm{~mm}$

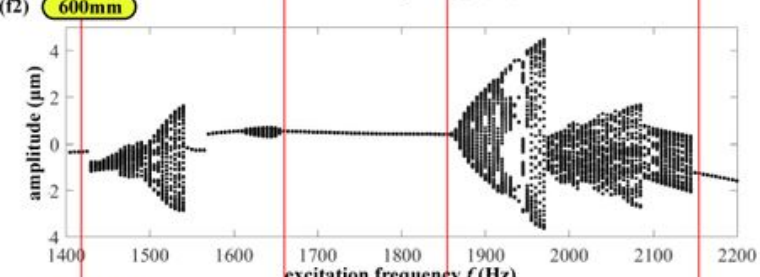

(g2) $700 \mathrm{~mm}$

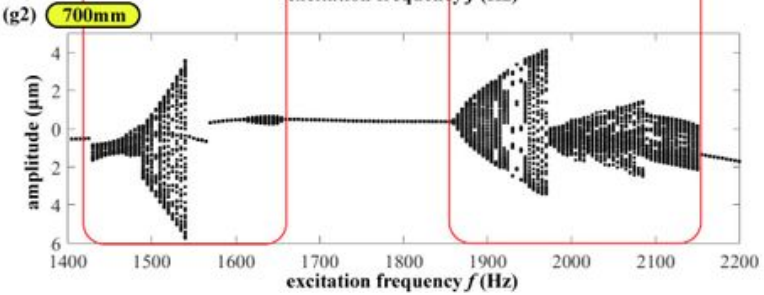

Figure 16

Bifurcation diagram with excitation frequency as the control parameter under different worktable position when excitation amplitude $\mathrm{E}=5000$ 
(a1) $100 \mathrm{~mm}$

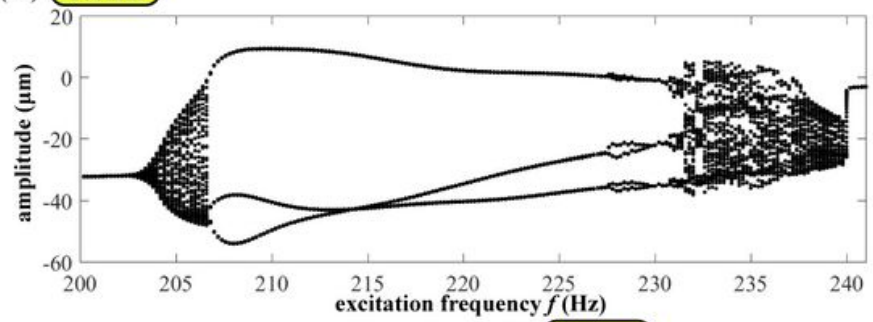

(b) $700 \mathrm{~mm}$

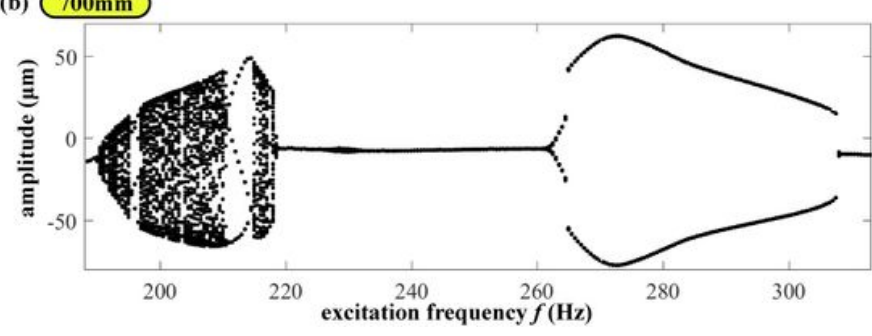

(a2) $100 \mathrm{~mm}$
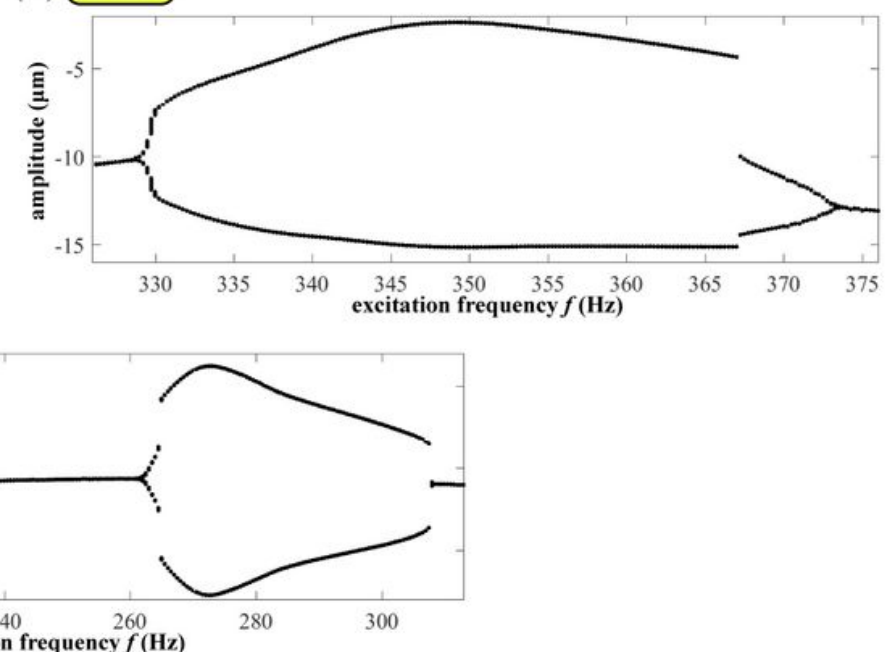

\section{Figure 17}

Partial bifurcation diagram with excitation frequency as the control parameter at $L p=100$ and $700 \mathrm{~mm}$ when $\mathrm{E}=5000$ 

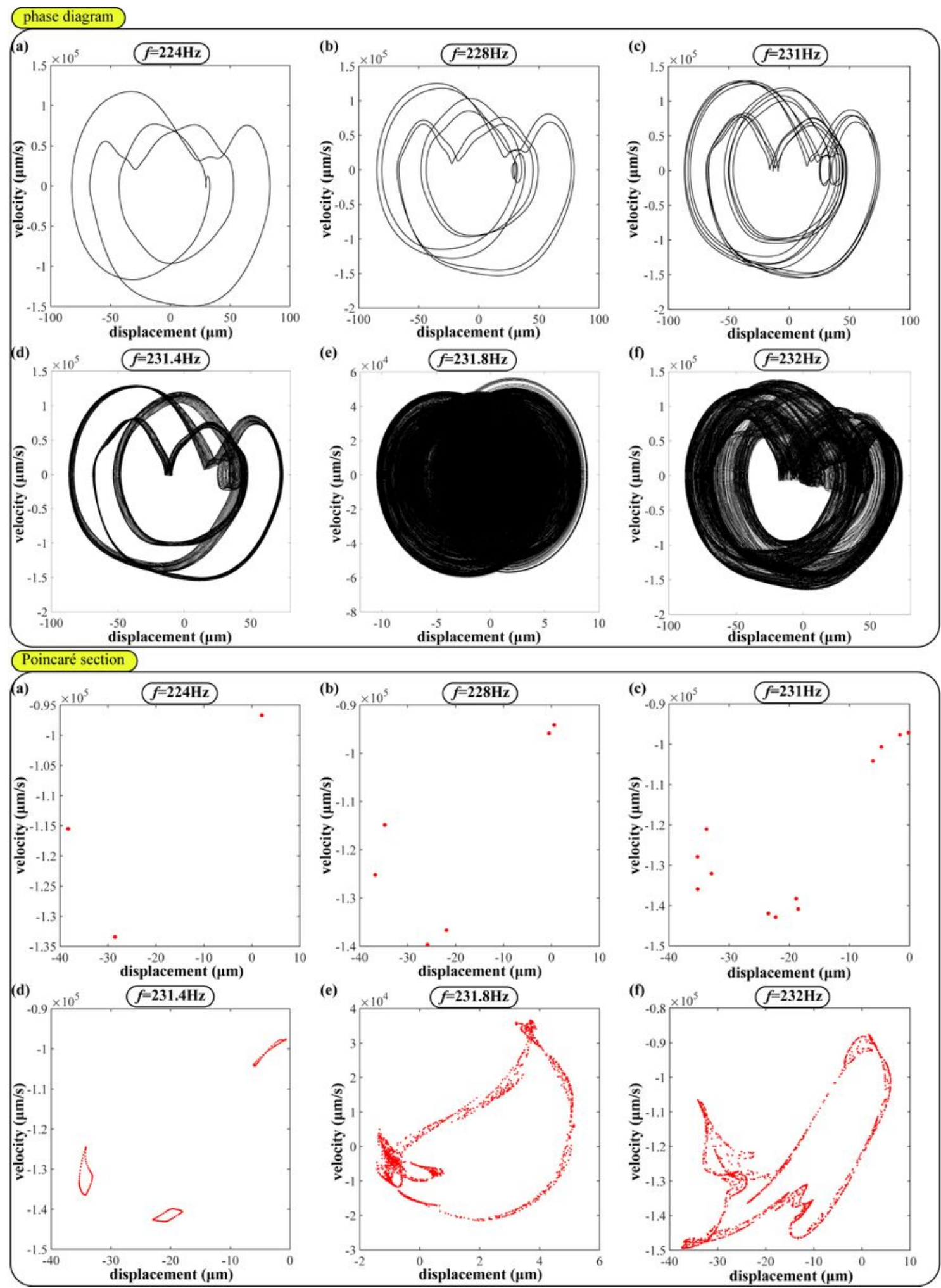

Figure 18

The evolution of orbit and Poincaré section under different excitation frequency when $L p=100 \mathrm{~mm}$ and $\mathrm{E}=5000$ 
(a)

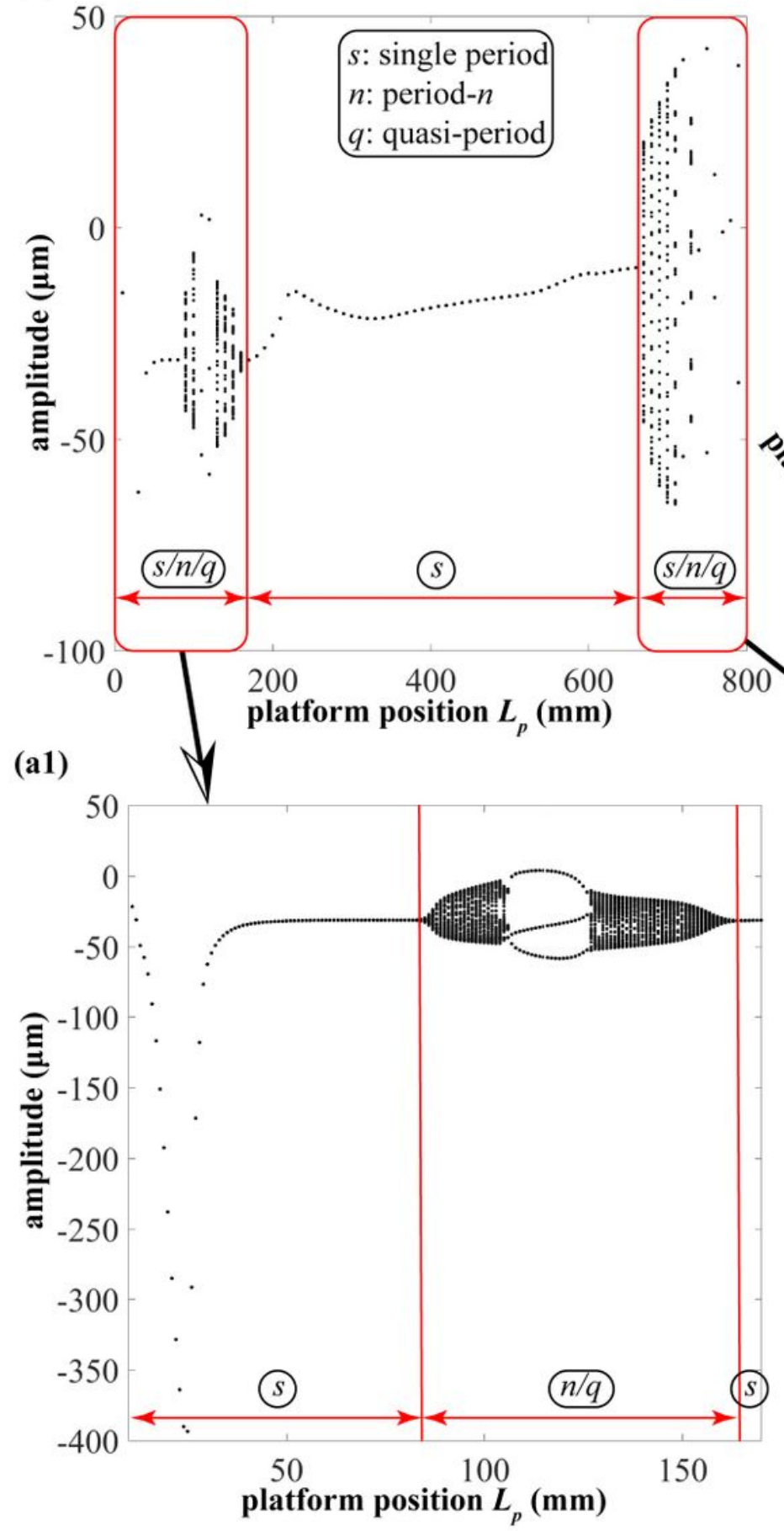

(b)

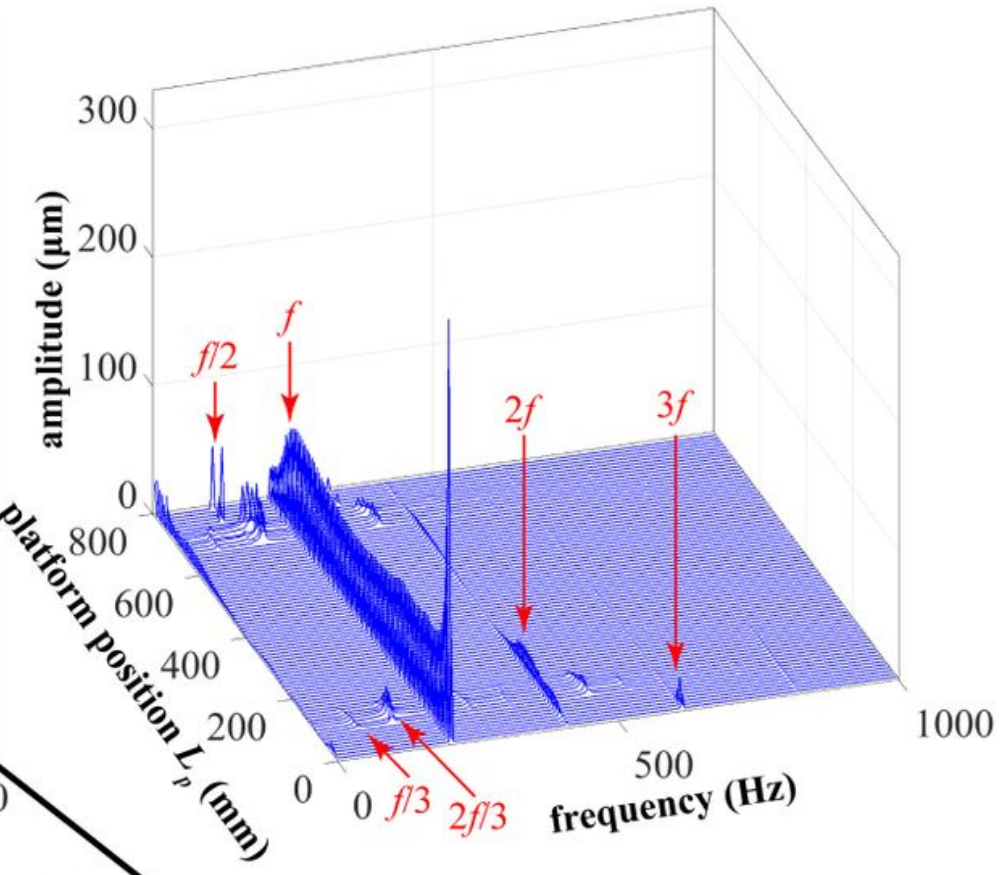

(a2)

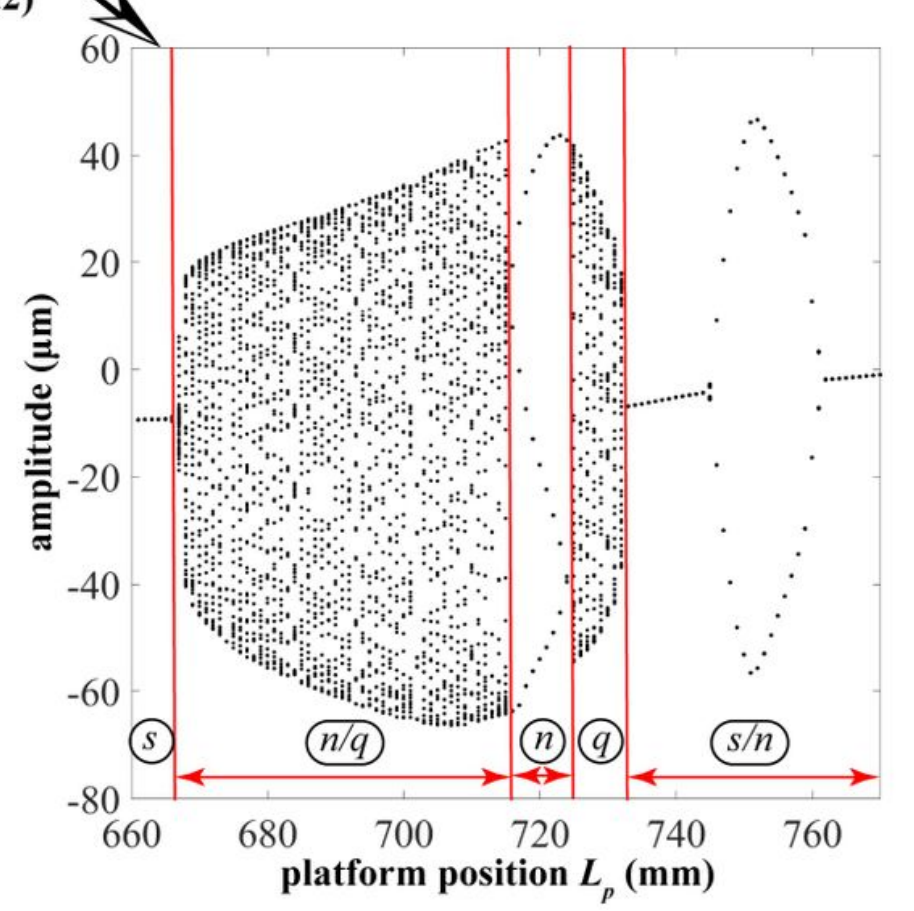

Figure 19

Bifurcation diagram and 3-D frequency spectrum with worktable position as the control parameter when excitation $\mathrm{f}=206 \mathrm{~Hz}$ and $\mathrm{E}=5000$ 


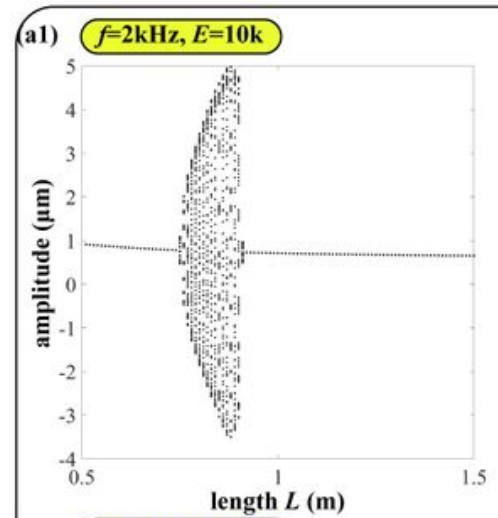

(a2) $f=2 \mathrm{kHz}, E=20 \mathrm{k}$

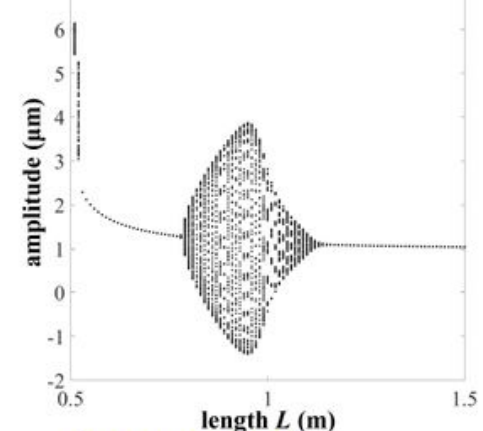

(b2) $f=2.37 \mathrm{kHz}, E=10 \mathrm{k}$

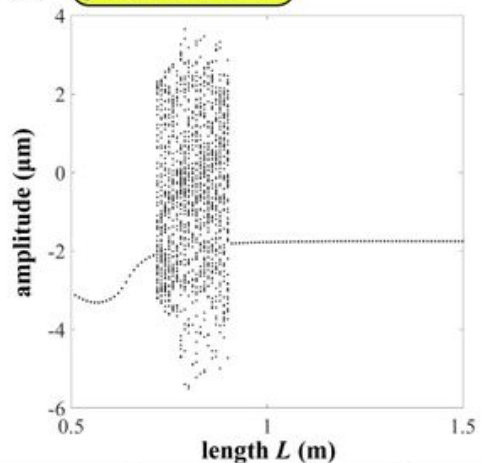

3-D frequency spectrum
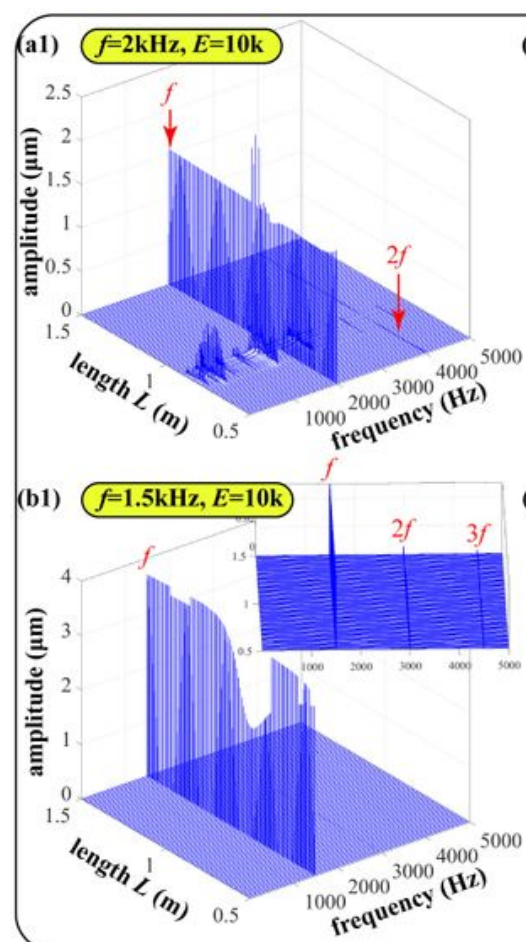

(a3) $f=2 \mathrm{kHz}, E=30 \mathrm{k}$

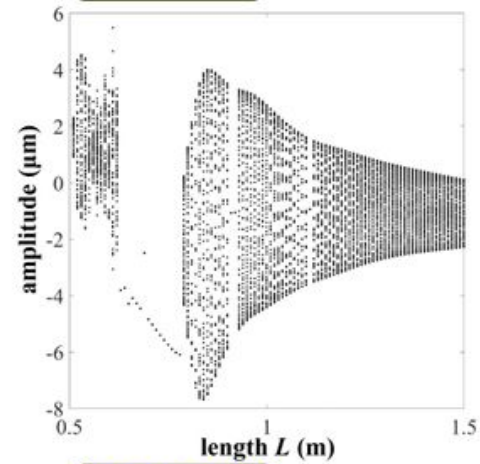

(b3) $f=2.5 \mathrm{kHz}, E=10 \mathrm{k}$
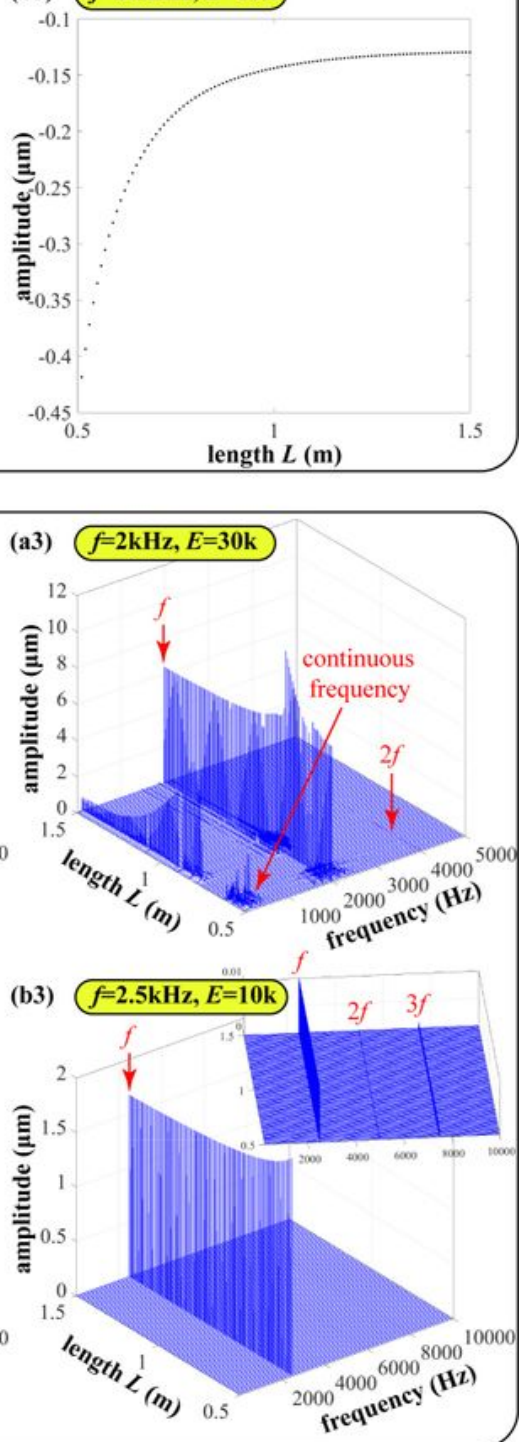

Figure 20

Bifurcation diagram and 3-D frequency spectrum with screw shaft length as the control parameter under different external excitation 

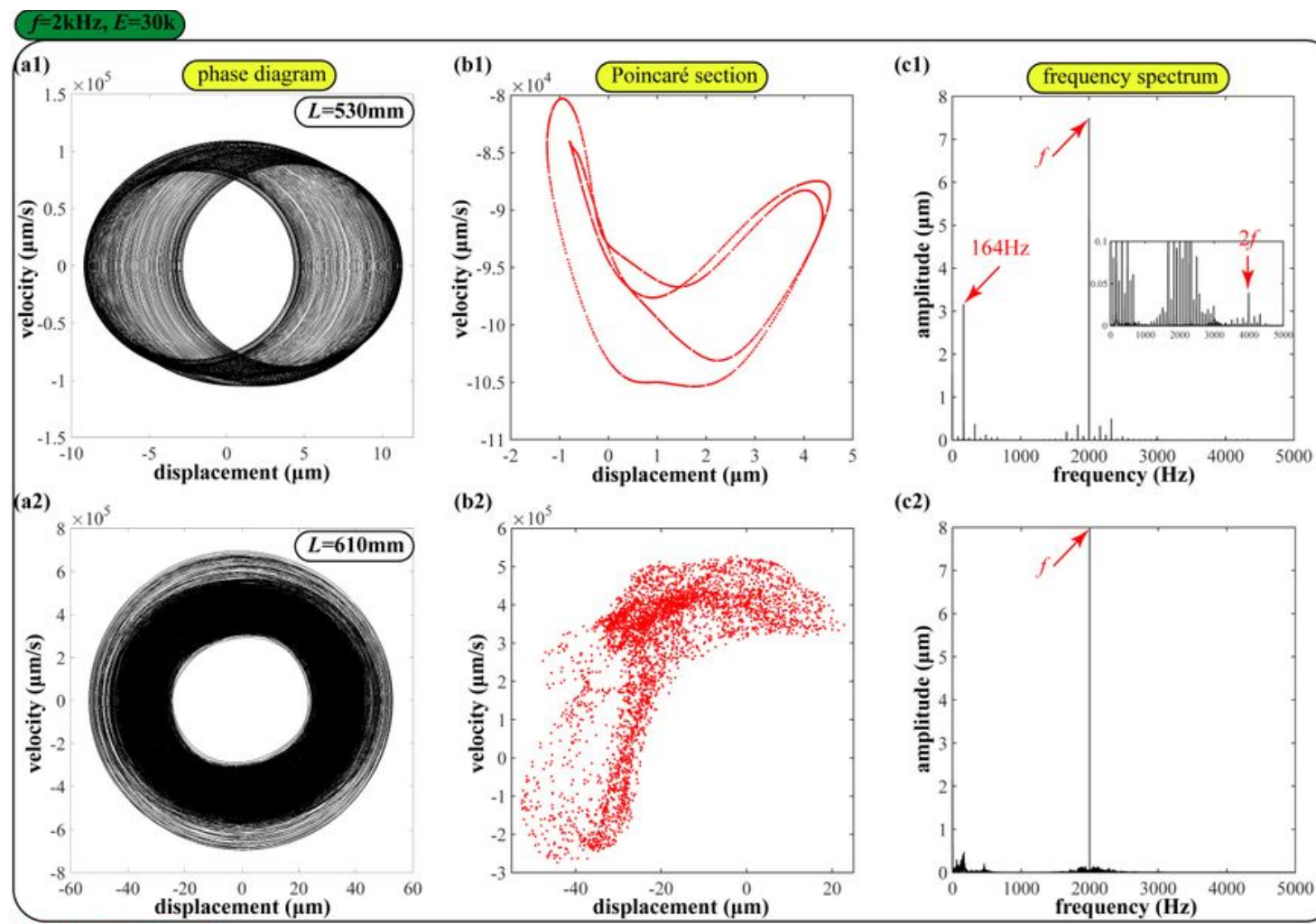

(c2)
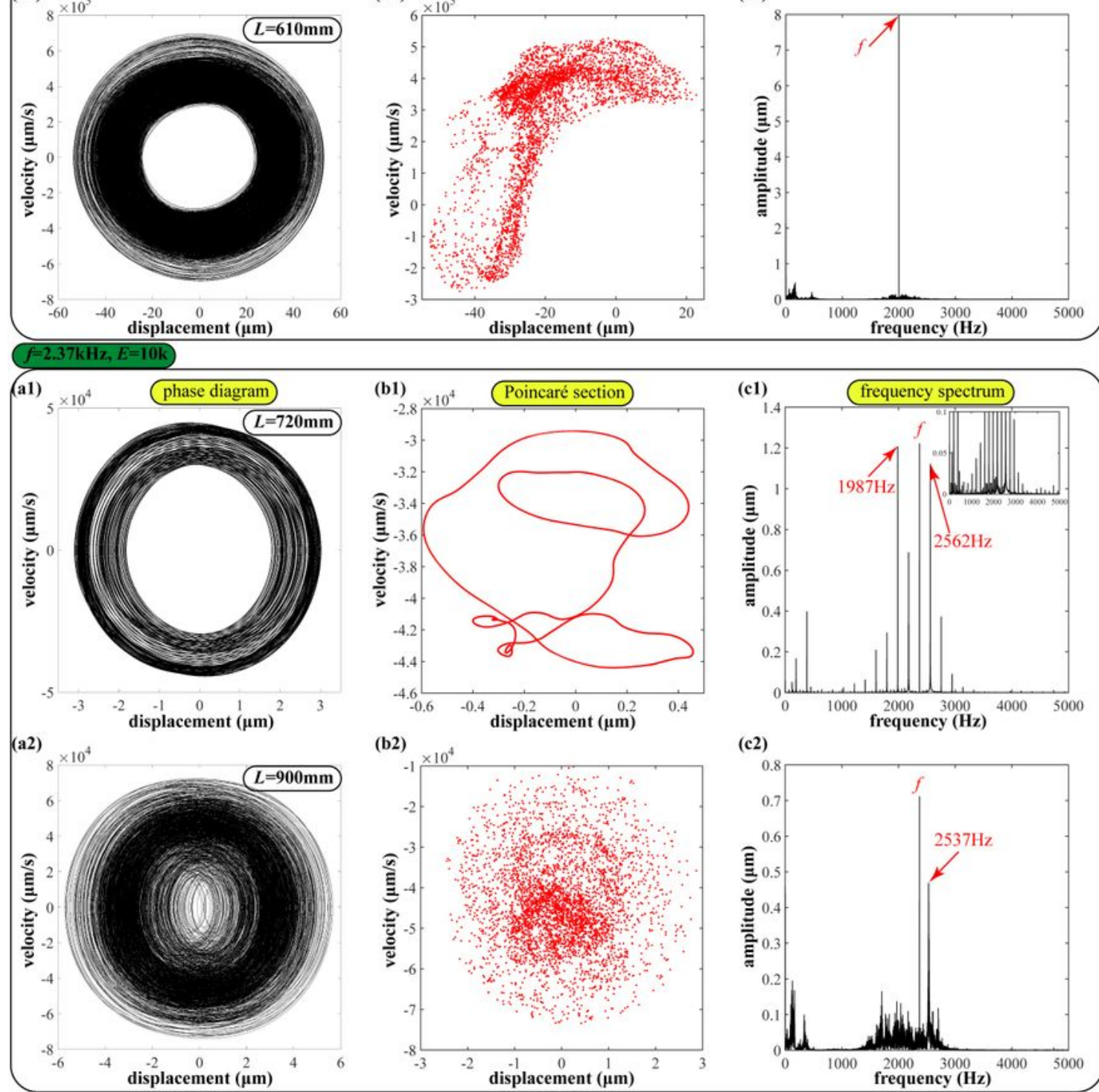

Figure 21

Vibration response under certain excitation and screw shaft length 
(a1) $E=10 \mathrm{k}$

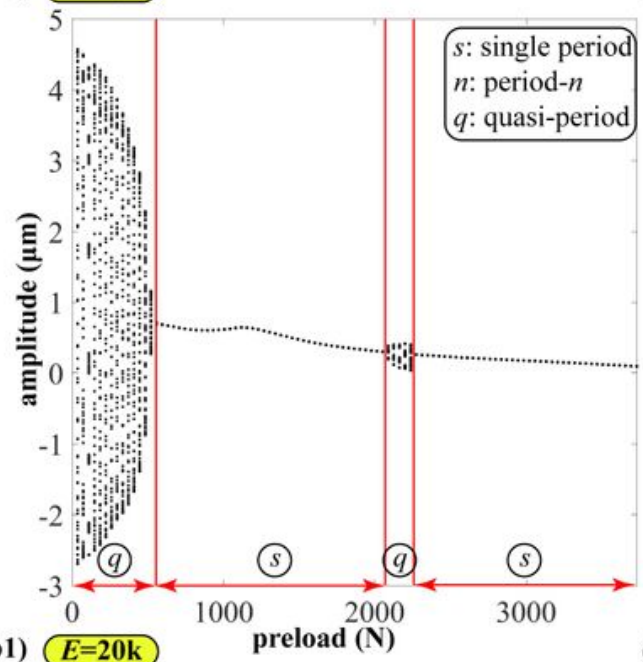

(b1) $E=20 \mathrm{k}$

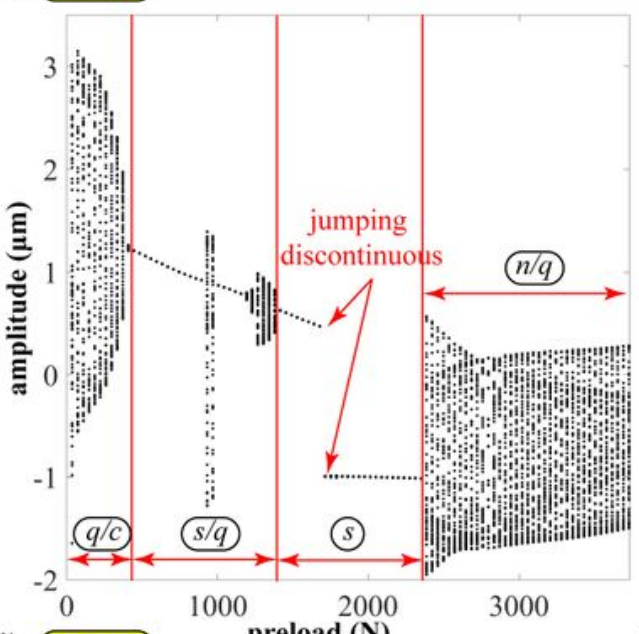

(c1) $E=30 \mathrm{k}$

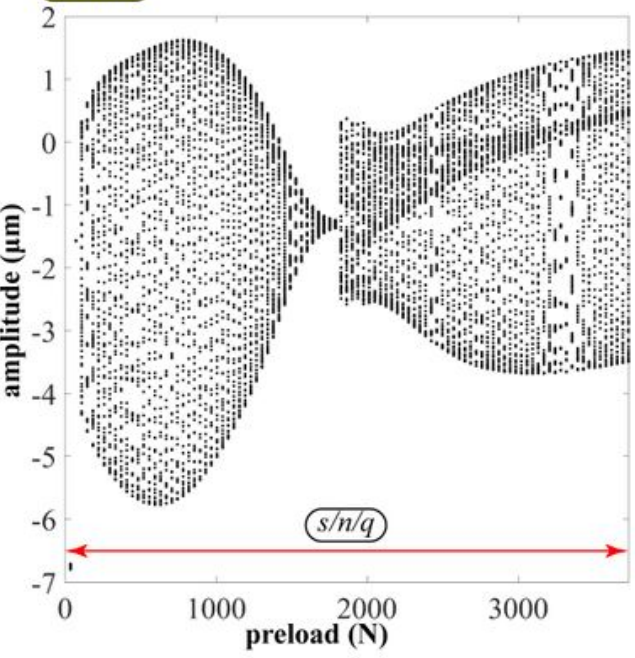

(a2)

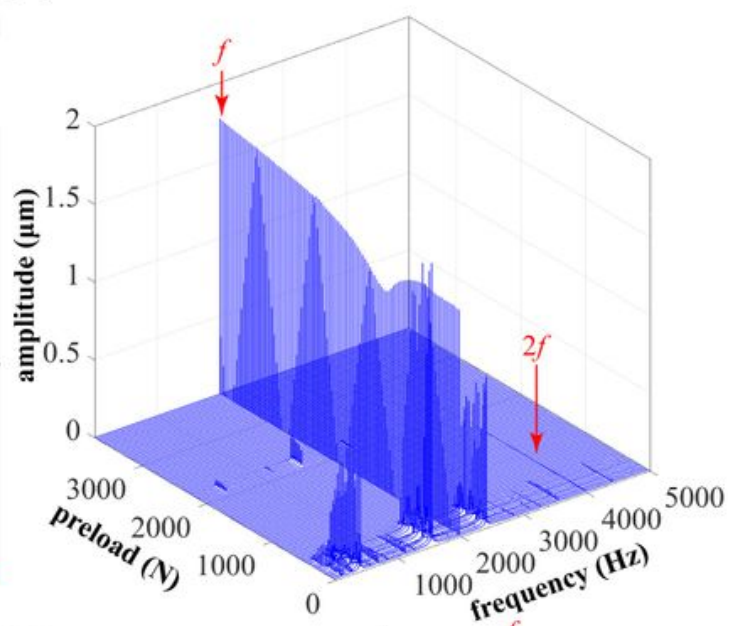

(b2)

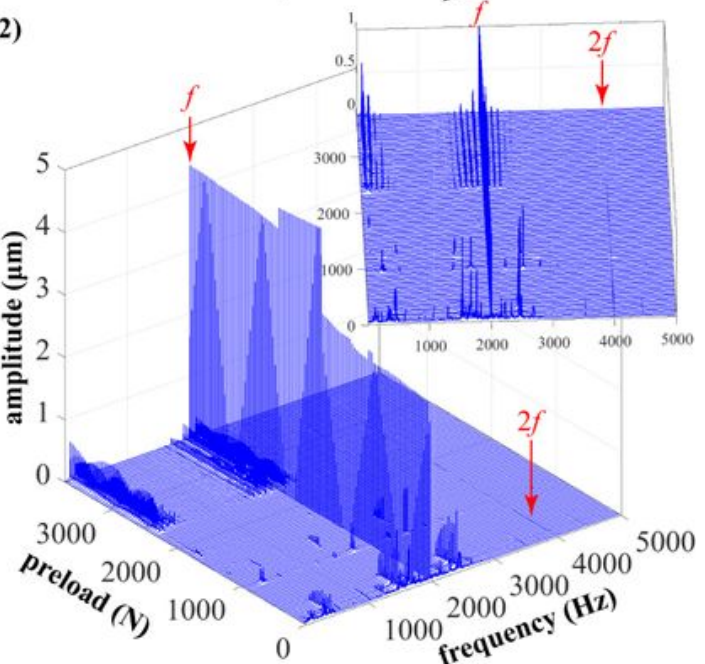

(c2)

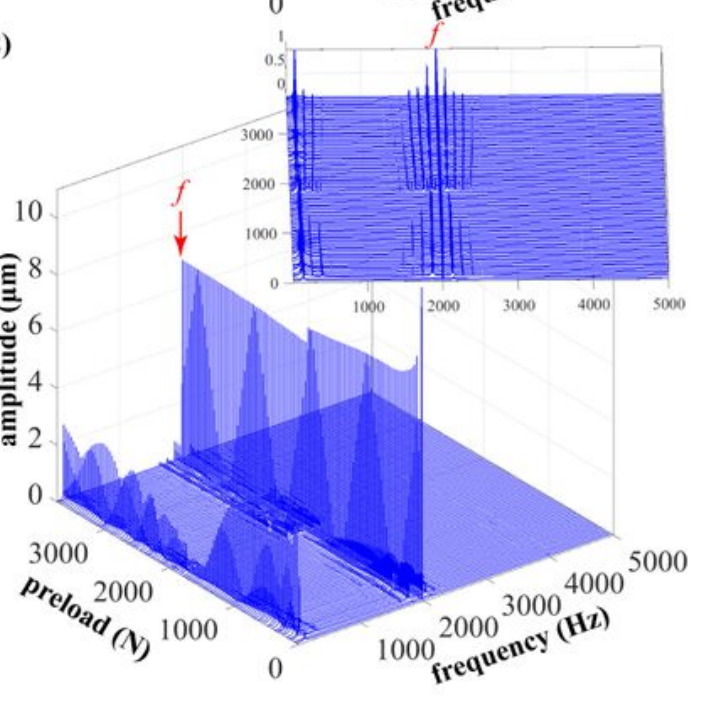

Figure 22

Bifurcation diagram and 3-D frequency spectrum with carriage preload as the control parameter under different excitation amplitide when $\mathrm{f}=2 \mathrm{kHz}$ 
(a1) $E=10 \mathrm{k}$
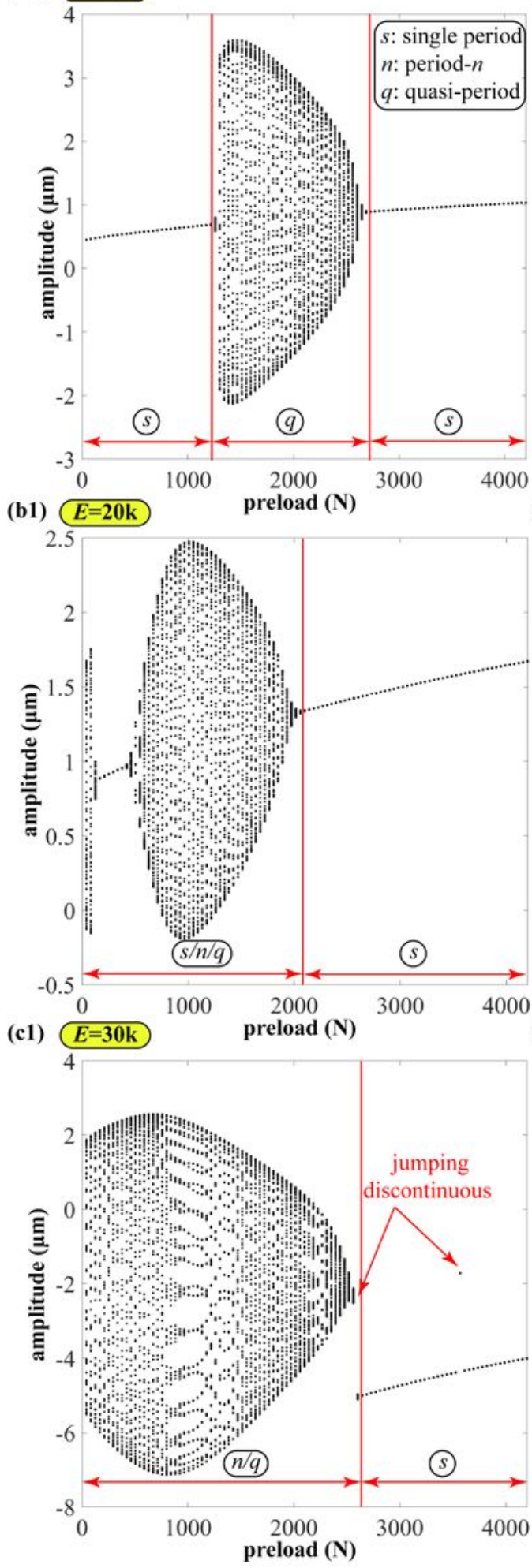

(c2)

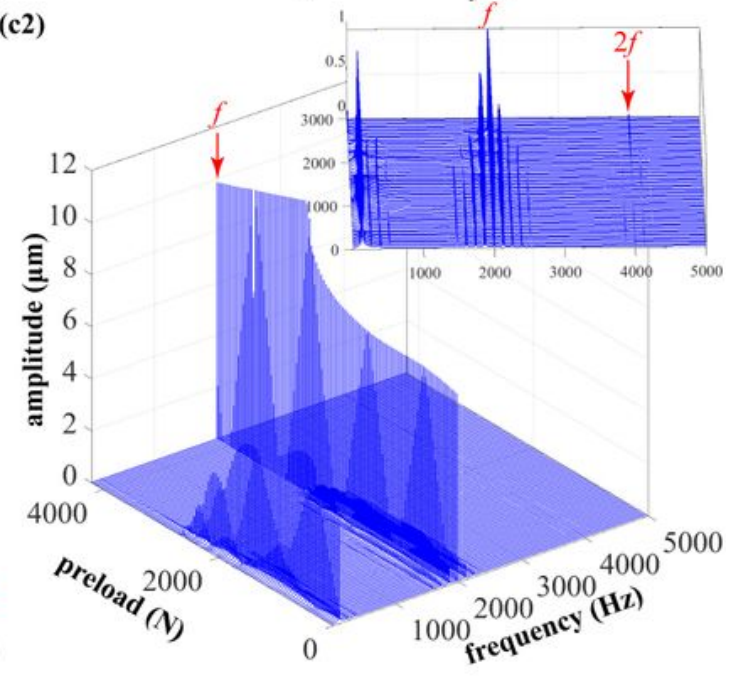

(a2)

(b2)
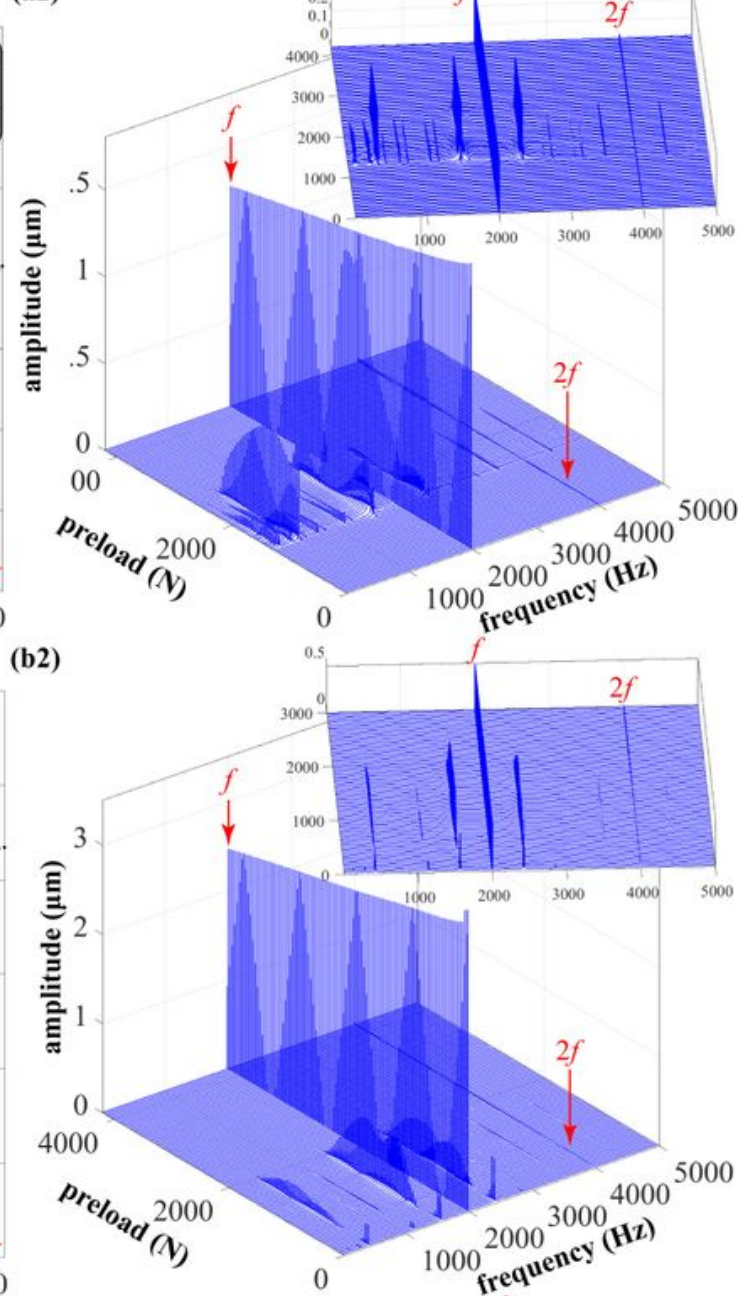

Figure 23

Bifurcation diagram and 3-D frequency spectrum with nut preload as the control parameter under different excitation amplitide when $\mathrm{f}=2 \mathrm{kHz}$ 


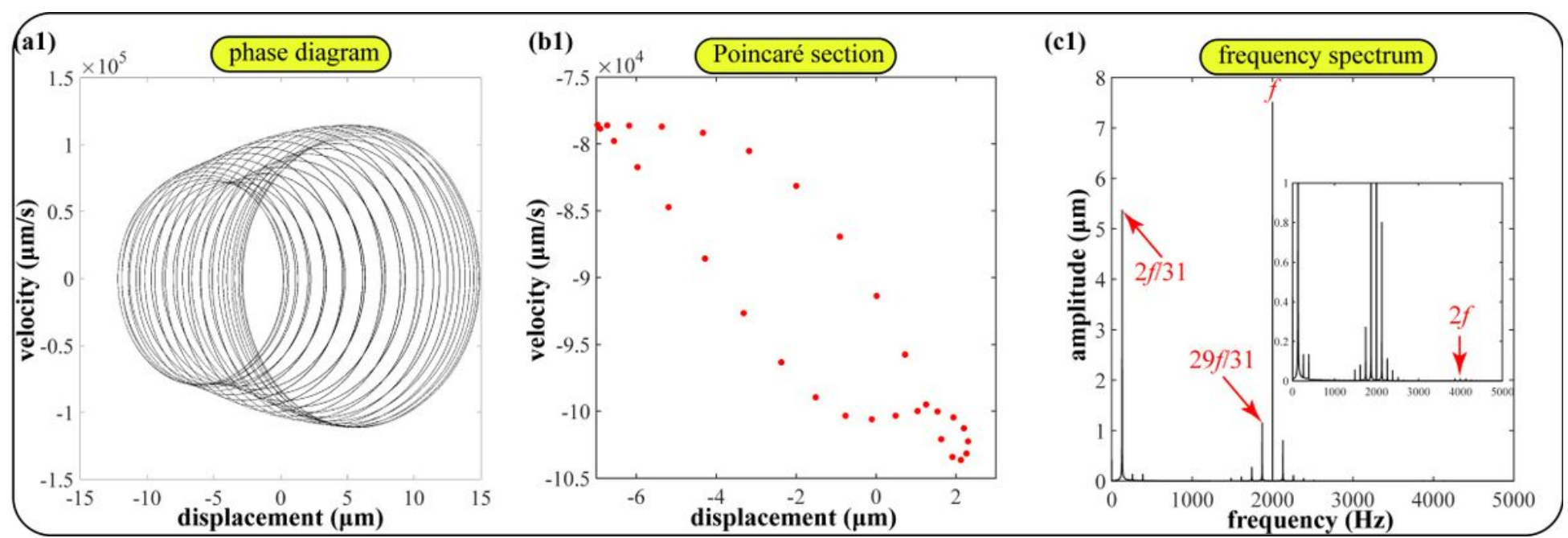

\section{Figure 24}

Vibration response of 31 T-period motion when excitation $f=2 k H z, E=30 k$ and nut preload is $1050 \mathrm{~N}$ 

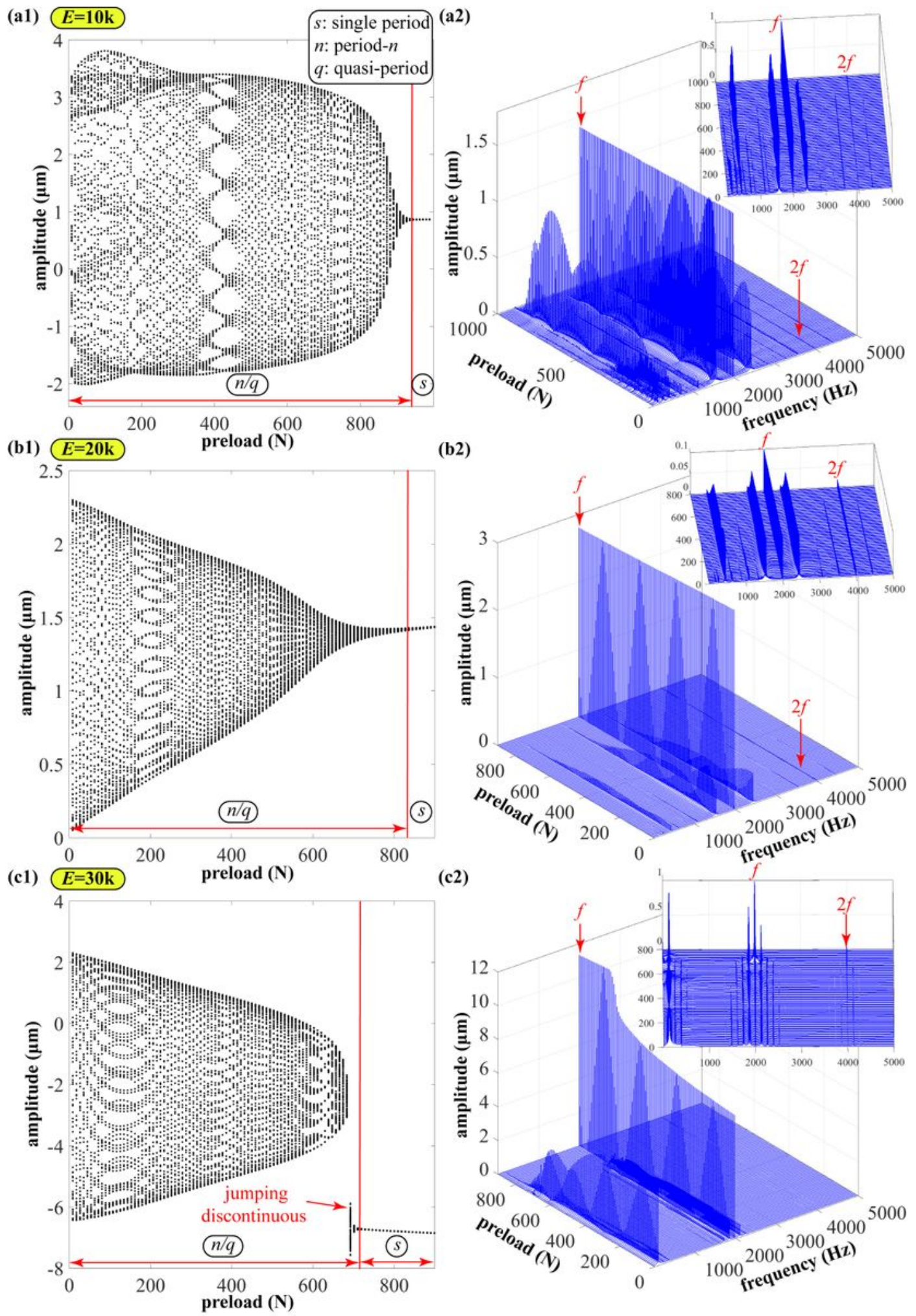

(b2)

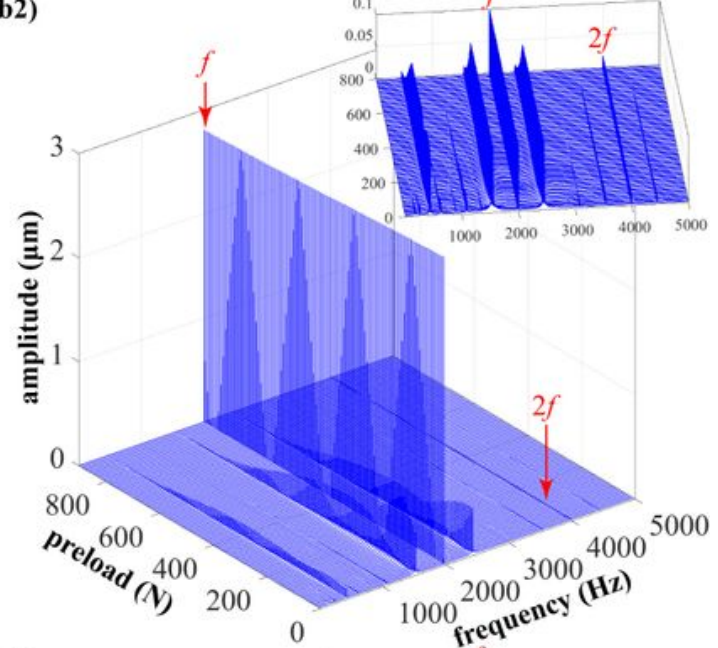

(c2)

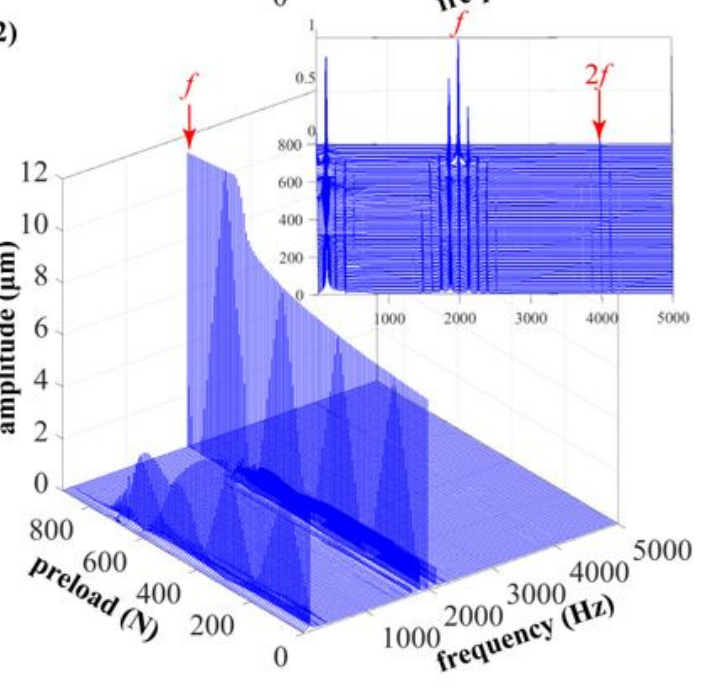

Figure 25

Bifurcation diagram and 3-D frequency spectrum with matched bearings preload as the control parameter under different excitation amplitide when $\mathrm{f}=2 \mathrm{kHz}$ 
(a1) carriage

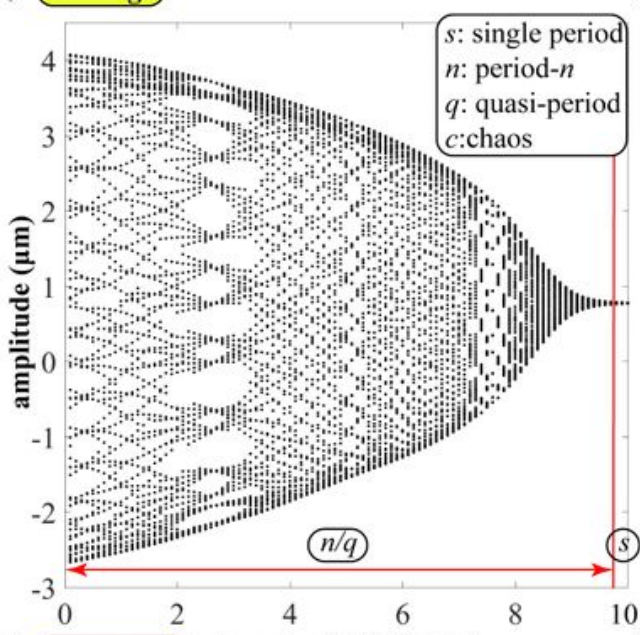

(b1) screw-nut damping $\left(\times 10^{3} \mathrm{~N} \cdot \mathrm{s} / \mathrm{m}\right)$

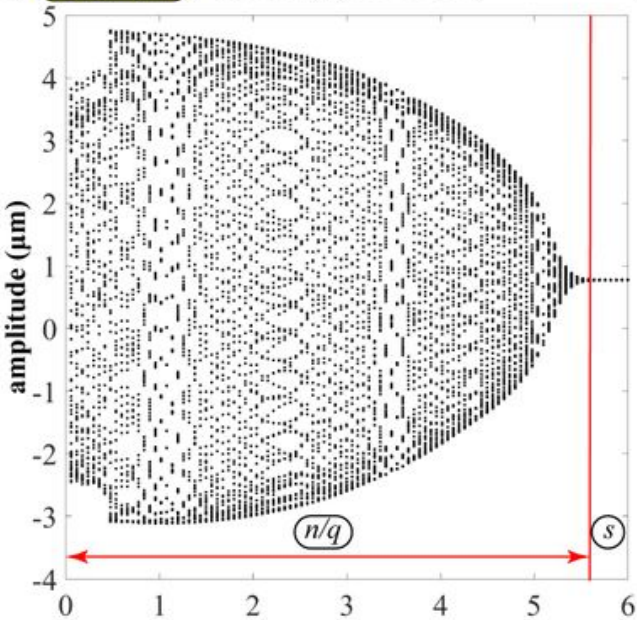

(c1) bearing damping $\left(\times 10^{3} \mathrm{~N} \cdot \mathrm{s} / \mathrm{m}\right)$

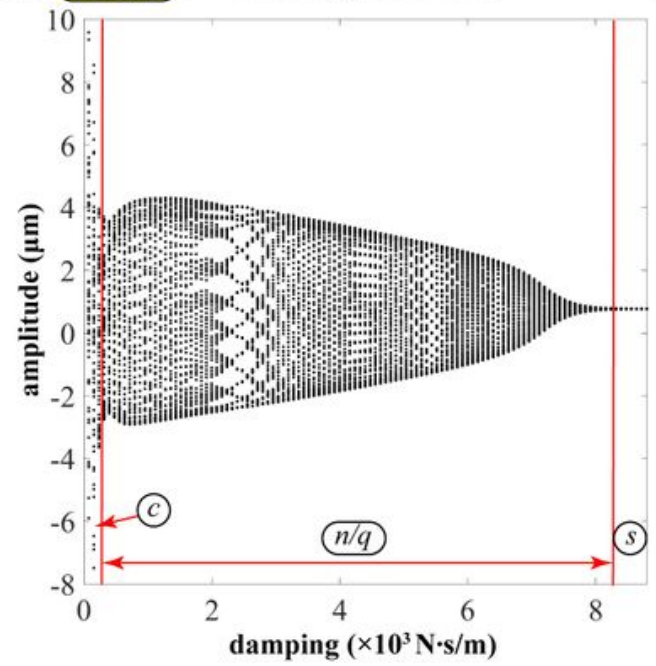

(a2)

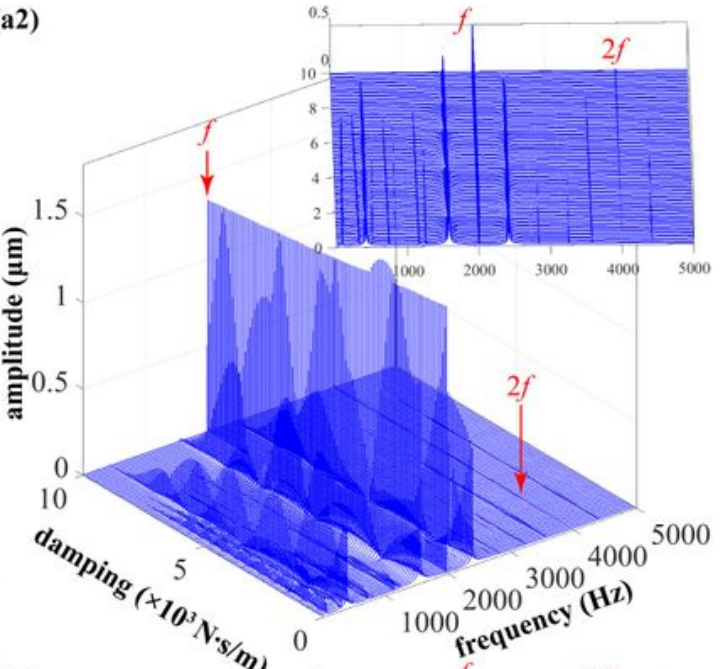

(b2)

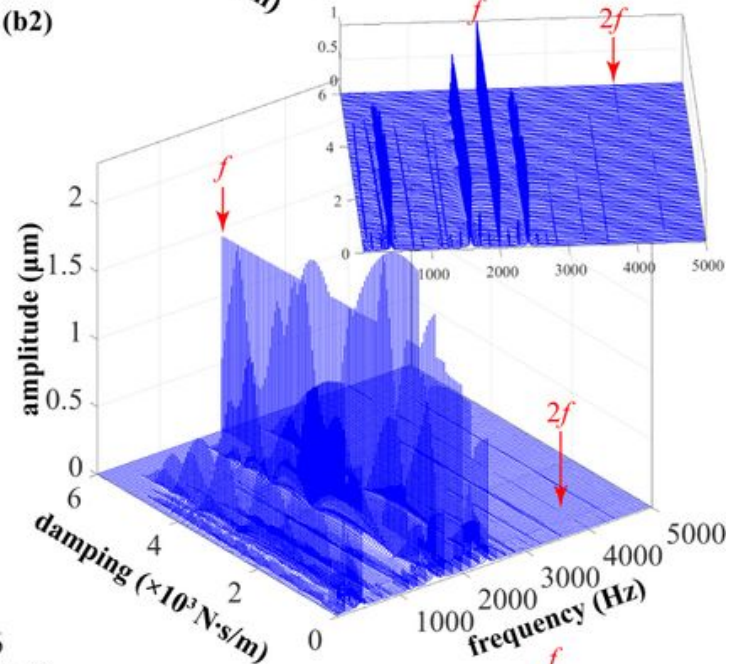

(c2)

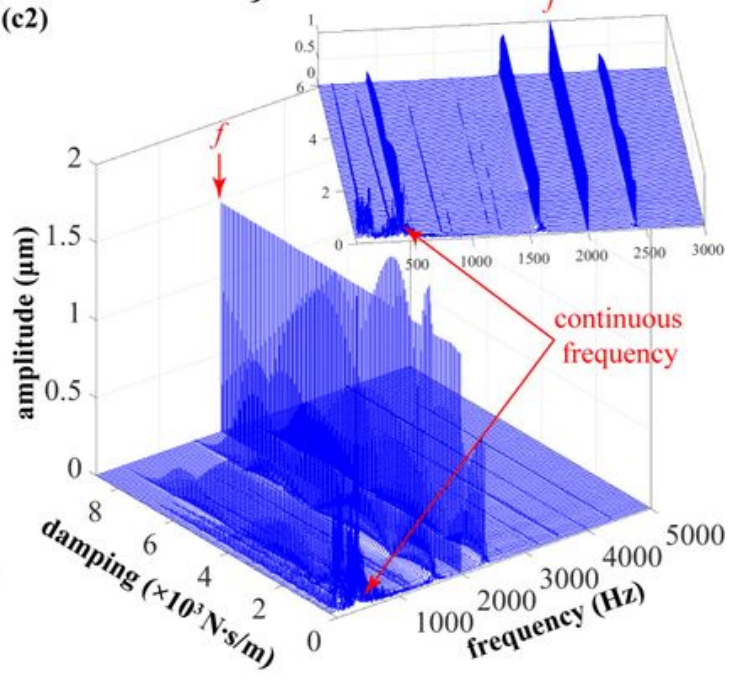

Figure 26

Bifurcation diagram and 3-D frequency spectrum with damping coefficient of rolling joints as the control parameter 

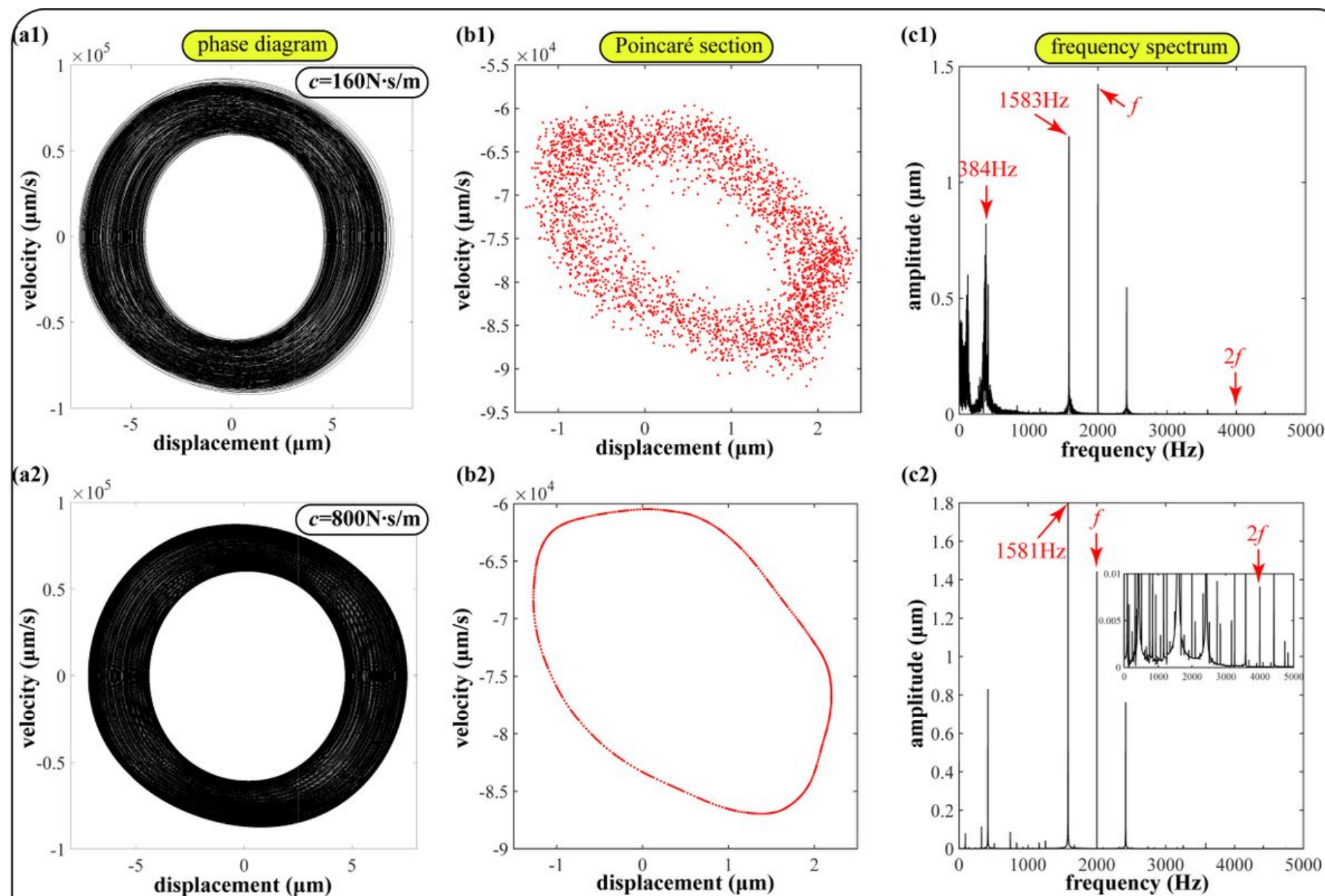

(c2)
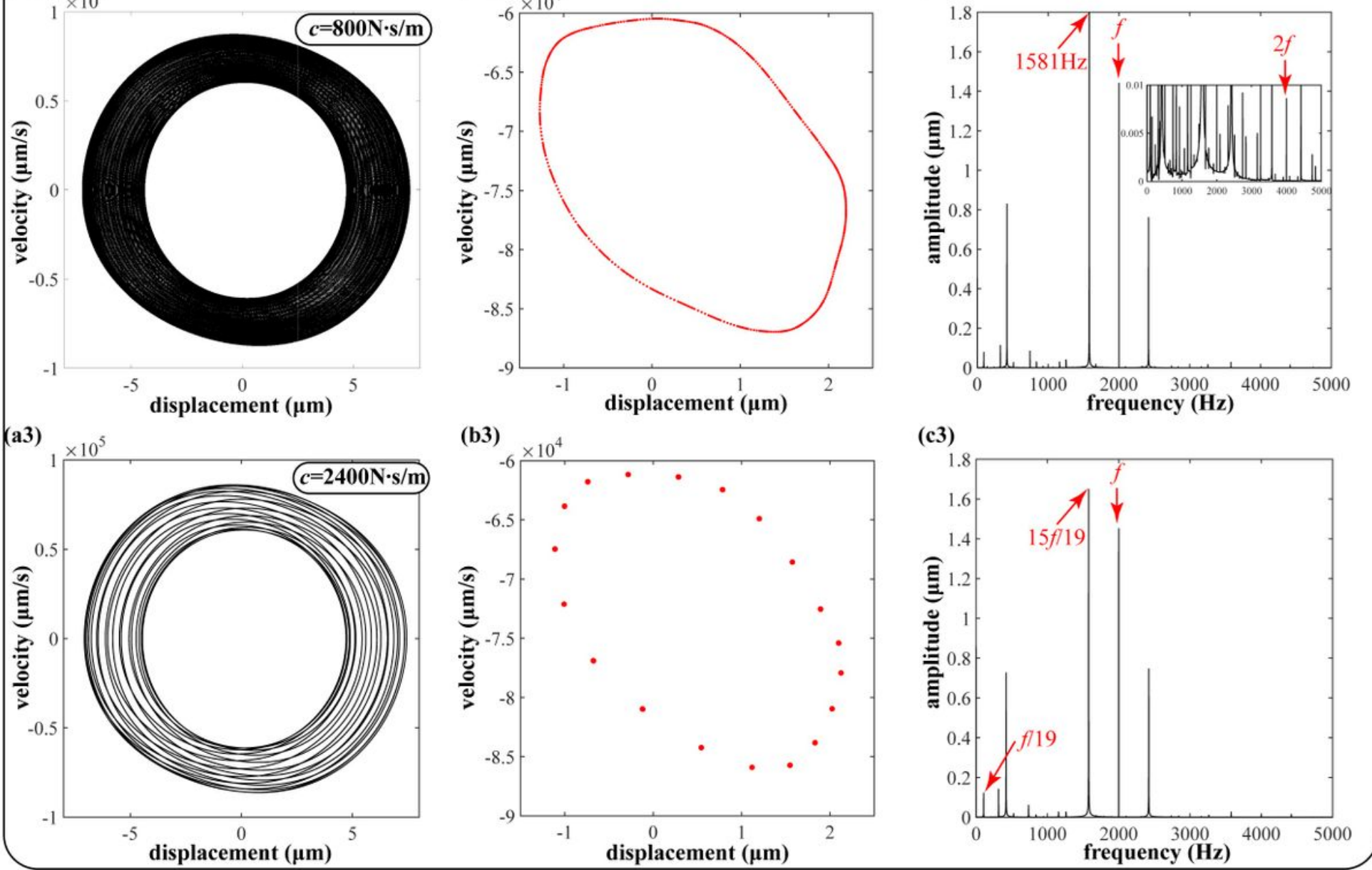

Figure 27

The evolution of detailed vibration response under different damping of matched bearings when $\mathrm{f}=2 \mathrm{kHz}$, $E=5000, L=800 \mathrm{~mm}, L p=400 \mathrm{~mm}$, preloads of carriage, nut and bearing are $623 \mathrm{~N}, 1690 \mathrm{~N}$ and $290 \mathrm{~N}$. 